Supporting information for:

A Novel Class of P-O Monophosphite Ligands Derived from

D-Mannitol: Broad Applications in Highly Enantioselective

\title{
Rh-Catalyzed Hydrogenations
}

Hanmin Huang, Zhuo Zheng ${ }^{*}$, Huili Luo, Changmin Bai, Xinquan Hu, and Huilin Chen

Dalian Institute of Chemical Physics, Chinese Academy of Sciences, Dalian, 116023, P. R. China

\section{CONTENTS}

(A) GC Analytical Data and graphic of Products (S1-S21)

(1) GC analytical data and graphic of hydrogenation products of dehydroamino acid esters (S2-S7).

(2) GC analytical data and graphic of hydrogenation products of enamides (S8-S16).

(3) GC analytical data and graphic of hydrogenation products of B-(acylamino)acrylates (S17-S20).

(4) GC analytical data and graphic of hydrogenation products of dimethyl itaconate (S20-S21).

(B) NMR of ligands 3a-k (S22-S54) 
(A) Determination of enantiomeric excesses of hydrogenation products

Gas chromatograms to determine enantiomeric excesses for $\alpha$-amino acid

esters

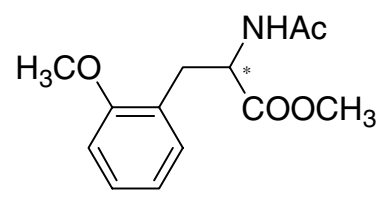

$97.4 \%$ ee $(\mathrm{S})$
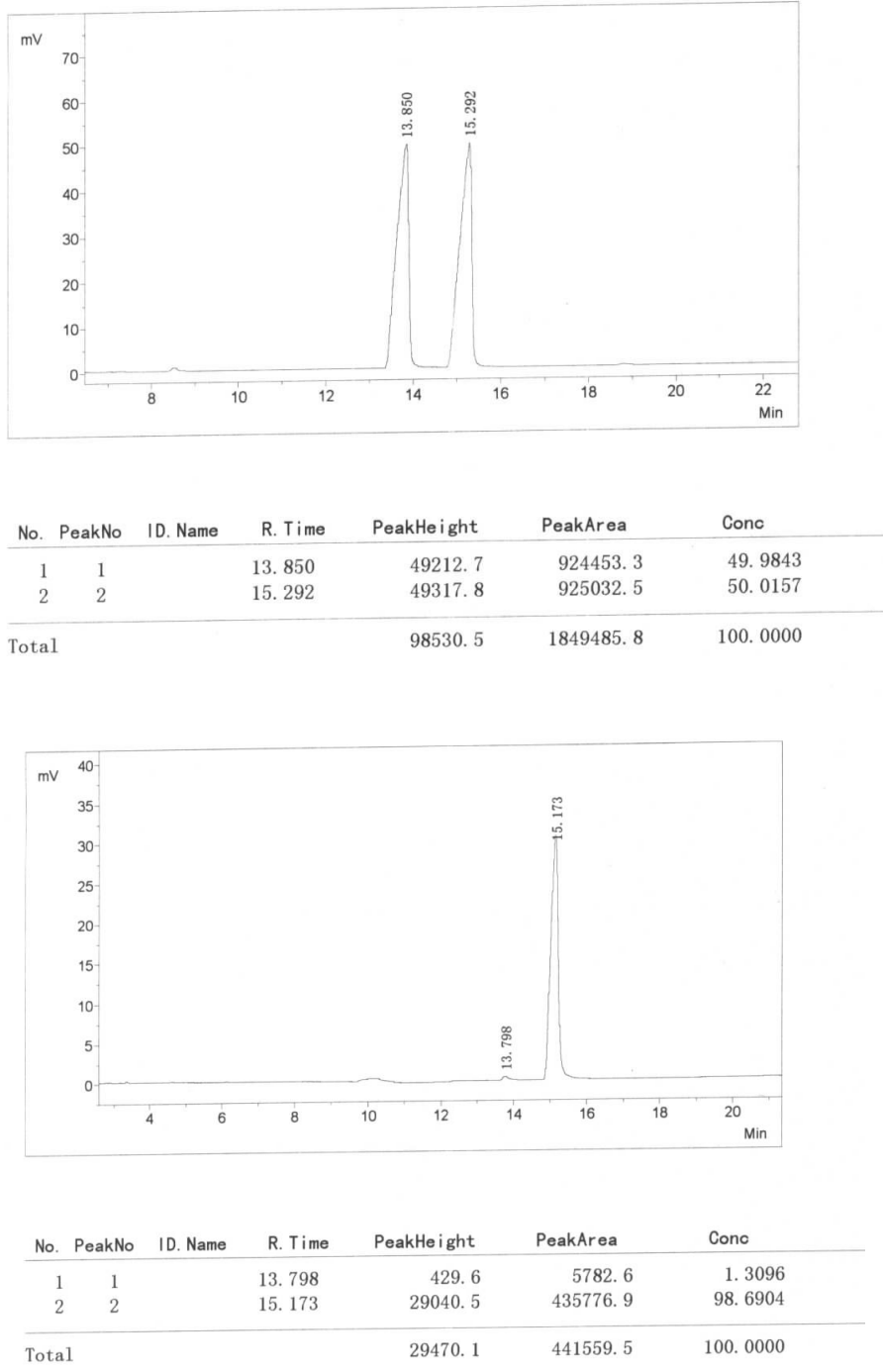

The ee's were determined by GC analysis using a Chrompack chiral fused silica $25 \mathrm{~m} \mathrm{x}$ $0.25 \mathrm{~mm}$ Chirasil-L-Val column. Oven temperature is $160{ }^{\circ} \mathrm{C}$. Absolute configuration was determined by comparison of the sign of the optical rotation with reported value. ${ }^{2}$ 


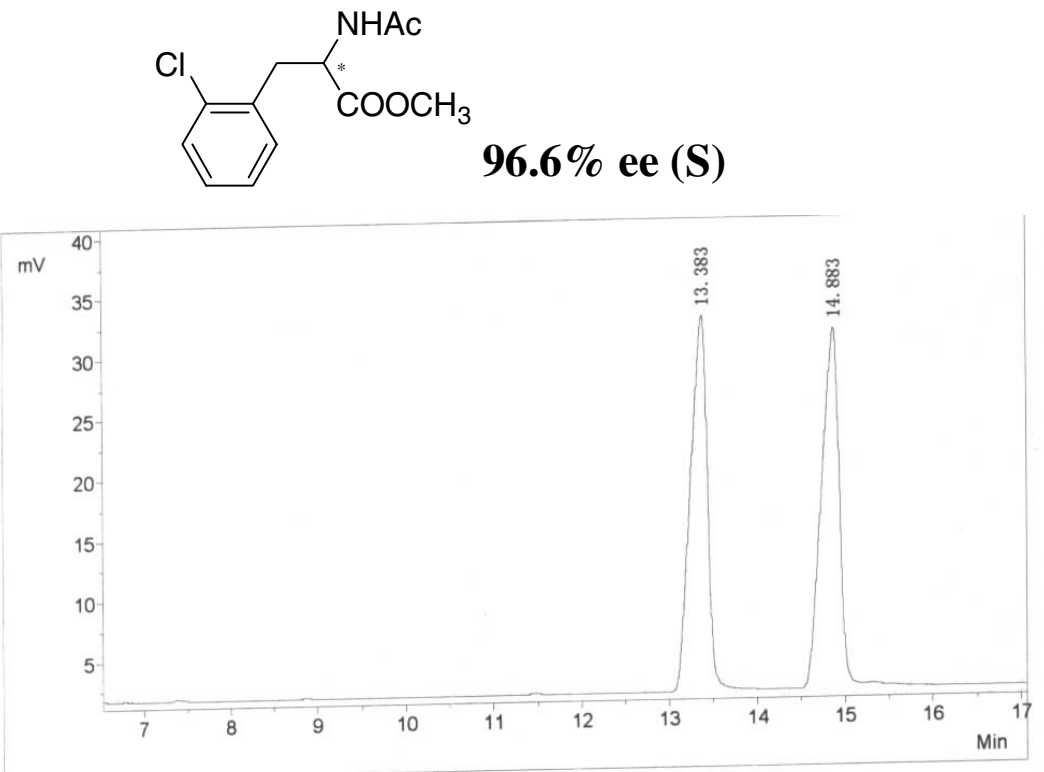

\begin{tabular}{|c|c|c|c|c|c|c|}
\hline No. & PeakNo & ID. Name & R. Time & PeakHe ight & PeakArea & Conc \\
\hline 1 & 1 & & 13. 383 & 31004.8 & 414951. 4 & 50. 0374 \\
\hline 2 & 2 & & 14. 883 & 29685.8 & 414330. 9 & 49. 9626 \\
\hline Total & & & & 60690.6 & 829282. 3 & 100.0000 \\
\hline
\end{tabular}

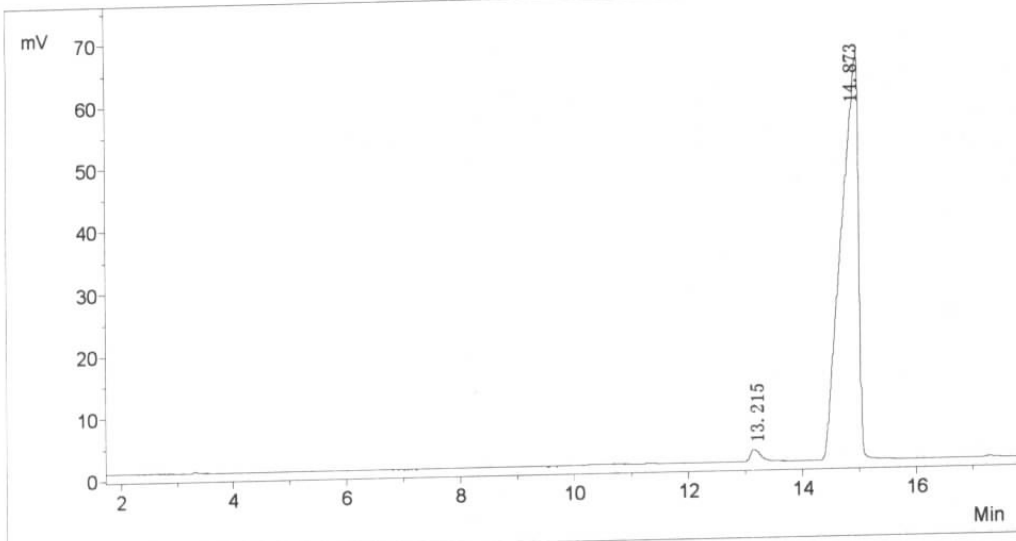

\begin{tabular}{rrrrrr} 
No. PeakNo & ID. Name & R. Time & PeakHeight & \multicolumn{1}{c}{ PeakArea } & \multicolumn{1}{c}{ Conc } \\
\hline 1 & 1 & 13.215 & 1615.7 & 23539.5 & 1.6975 \\
2 & 2 & 14.873 & 55865.8 & 1363169.1 & 98.3025 \\
\hline \multicolumn{2}{r}{ Total } & & 57481.5 & 1386708.6 & 100.0000
\end{tabular}

The ee's were determined by GC analysis using a Chrompack chiral fused silica $25 \mathrm{~m} \mathrm{x}$ $0.25 \mathrm{~mm}$ chirasil-L-Val column. Oven temperature is $160{ }^{\circ} \mathrm{C}$. Absolute configuration was determined by comparison of the sign of the optical rotation with reported value. ${ }^{2}$ 


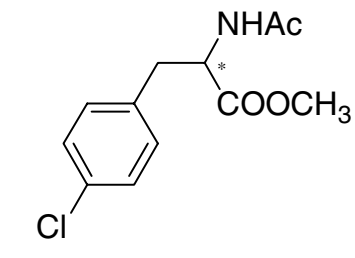

$97.1 \%$ ee (S)

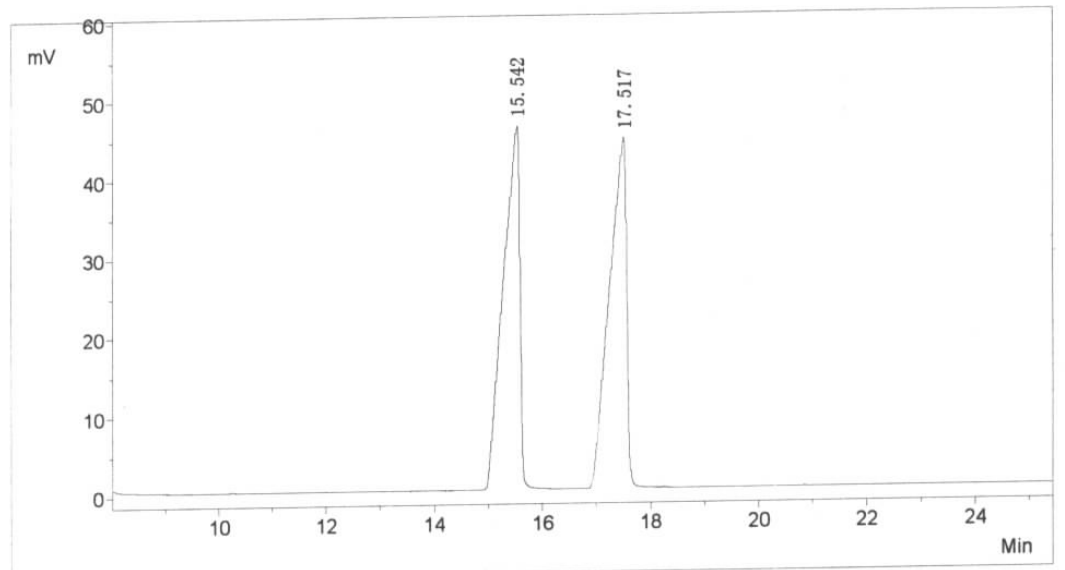

\begin{tabular}{|c|c|c|c|c|c|c|}
\hline No. & PeakNo & ID. Name & R. Time & PeakHeight & PeakArea & Conc \\
\hline 1 & 1 & & 15. 542 & 45915.9 & 978946.3 & 49. 9581 \\
\hline 2 & 2 & & 17.517 & 44190.3 & 980589.6 & 50. 0419 \\
\hline Total & & & & 90106.2 & 1959535. 9 & 100.0000 \\
\hline
\end{tabular}

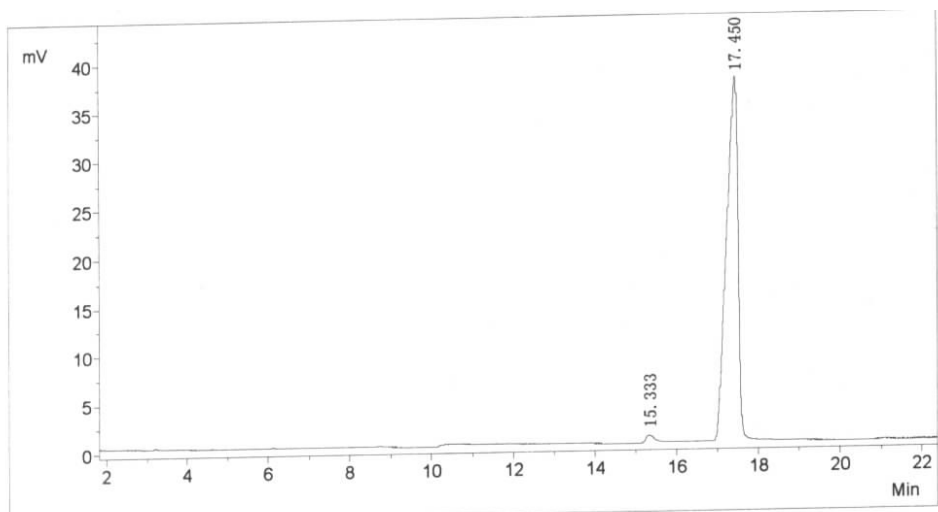

\begin{tabular}{rrrrrr} 
No. PeakNo & ID. Name & R. Time & PeakHeight & \multicolumn{1}{c}{ PeakArea } & \multicolumn{1}{c}{ Conc } \\
\hline 1 & 1 & 15.333 & 767.1 & 10465.5 & 1.4205 \\
2 & 2 & 17.450 & 37460.1 & 726267.9 & 98.5795 \\
\hline Total & & 38227.3 & 736733.4 & 100.0000
\end{tabular}

The ee's were determined by GC analysis using a Chrompack chiral fused silica $25 \mathrm{~m} \mathrm{x}$ $0.25 \mathrm{~mm}$ chirasil-L-Val column. Oven temperature is $160{ }^{\circ} \mathrm{C}$. Absolute configuration was determined by comparison of the sign of the optical rotation with reported value. ${ }^{2}$ 

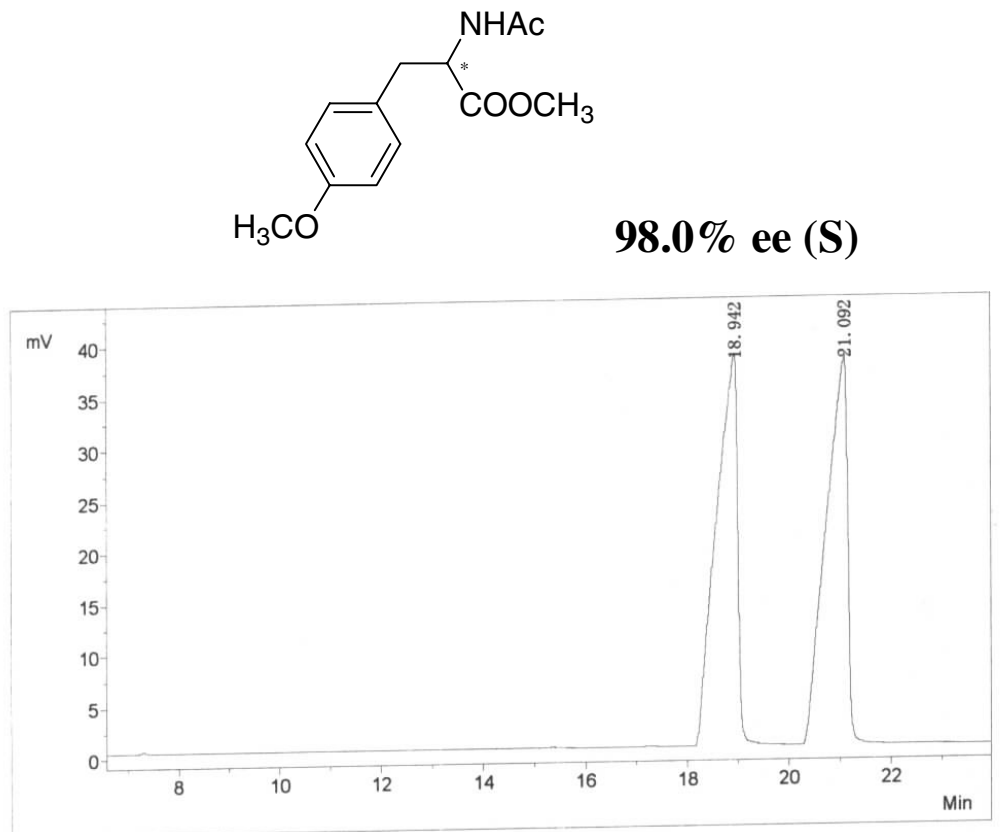

$98.0 \%$ ee (S)

\begin{tabular}{ccrrrr} 
No. PeakNo & ID. Name & R. Time & PeakHeight & PeakArea & \multicolumn{1}{c}{ Conc } \\
\hline 1 & 1 & 18.942 & 37876.9 & 1091534.7 & 49.9581 \\
2 & 2 & 21.092 & 37863.0 & 1093367.5 & 50.0419 \\
\hline Total & & 75739.9 & 2184902.2 & 100.0000
\end{tabular}

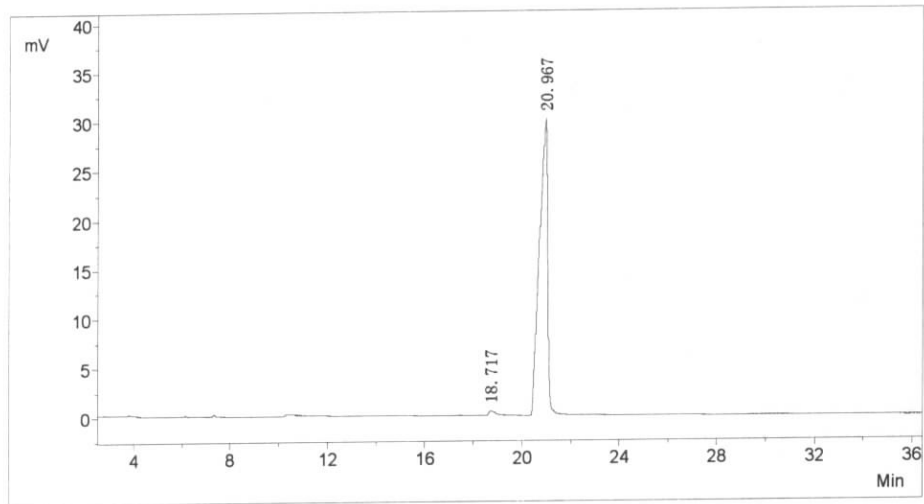

\begin{tabular}{rrrrrr} 
No. PeakNo & ID. Name & R. Time & PeakHeight & \multicolumn{1}{c}{ PeakArea } & \multicolumn{1}{c}{ Conc } \\
\hline 1 & 1 & 18.717 & 456.4 & 7363.9 & 1.0011 \\
2 & 2 & 20.967 & 30081.1 & 728182.3 & 98.9989 \\
\hline Total & & 30537.5 & 735546.2 & 100.0000
\end{tabular}

The ee's were determined by GC analysis using a Chrompack chiral fused silica $25 \mathrm{~m} \mathrm{x}$ $0.25 \mathrm{~mm}$ chirasil-L-Val column. Oven temperature is $160{ }^{\circ} \mathrm{C}$. Absolute configuration was determined by comparison of the sign of the optical rotation with reported value. ${ }^{2}$ 


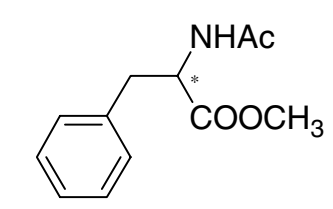

96.8\% ee (S)

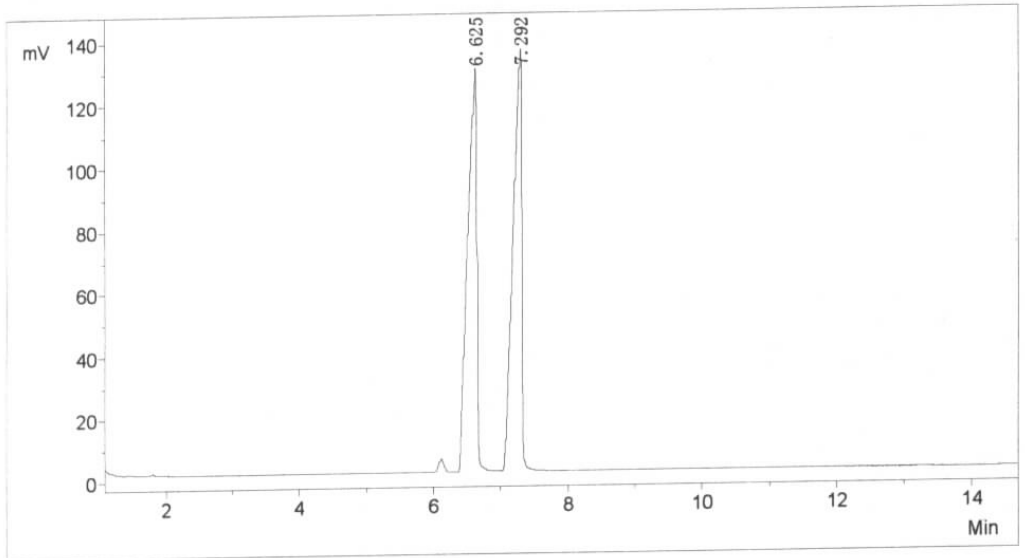

\begin{tabular}{ccrrrc} 
No. PeakNo & ID. Name & R. Time & PeakHeight & \multicolumn{1}{c}{ PeakArea } & \multicolumn{1}{c}{ Conc } \\
\hline 1 & 1 & 6.625 & 127435.5 & 1216486.3 & 50.0248 \\
2 & 2 & 7.292 & 134030.2 & 1215279.1 & 49.9752 \\
\hline Total & & & 261465.7 & 2431765.4 & 100.0000
\end{tabular}

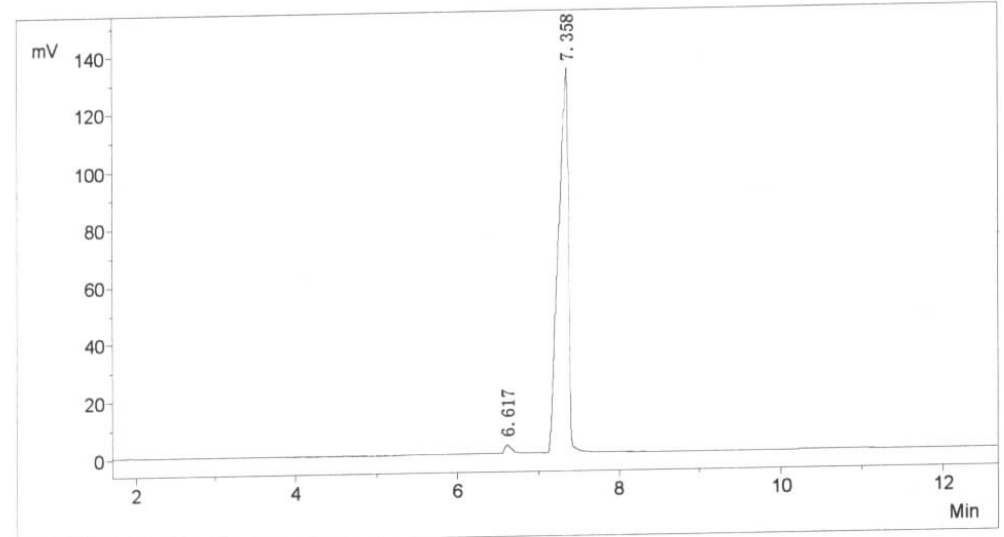

\begin{tabular}{rrrrrr} 
No. PeakNo & ID. Name & R. Time & PeakHeight & \multicolumn{1}{c}{ PeakArea } & \multicolumn{1}{c}{ Conc } \\
\hline 1 & 1 & 6.617 & 2883.3 & 18551.9 & 1.6037 \\
2 & 2 & 7.358 & 133246.6 & 1138232.9 & 98.3963 \\
\hline Total & & 136130.0 & 1156784.7 & 100.0000
\end{tabular}

The ee's were determined by GC analysis using a Chrompack chiral fused silica $25 \mathrm{~m} \mathrm{x}$ $0.25 \mathrm{~mm}$ chirasil-L-Val column. Oven temperature is $160{ }^{\circ} \mathrm{C}$. Absolute configuration was determined by comparison of the sign of the optical rotation with reported value. ${ }^{2}$ 

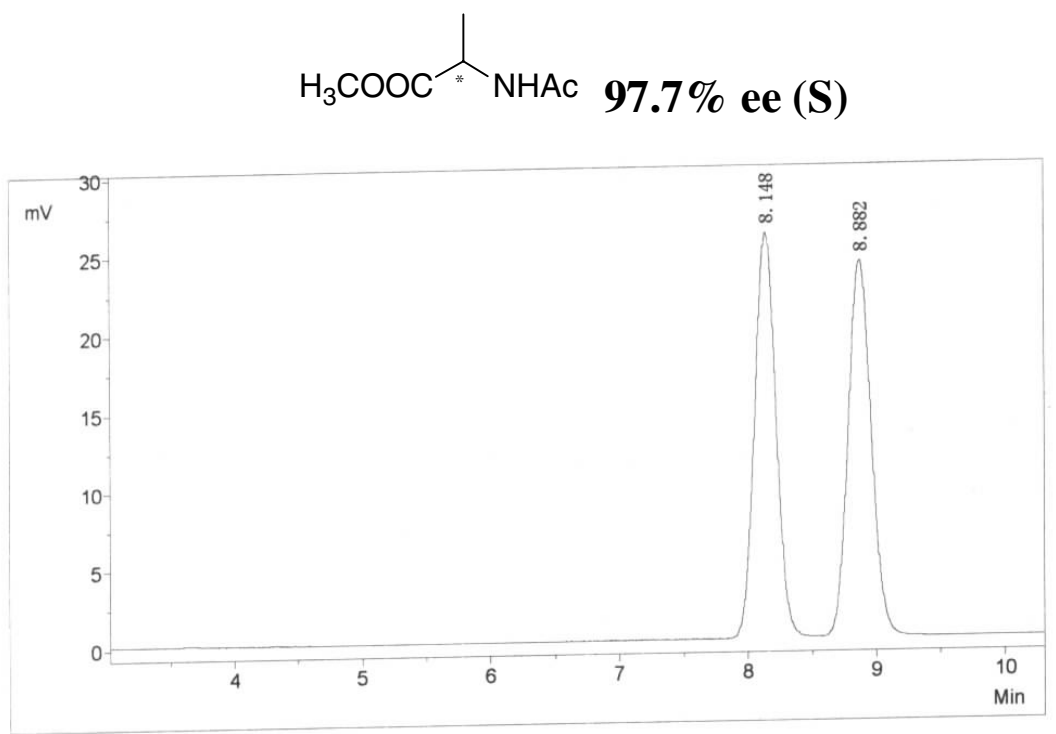

\begin{tabular}{|c|c|c|c|c|c|c|}
\hline No. & PeakNo & ID. Name & R. Time & PeakHeight & PeakArea & Conc \\
\hline 1 & 1 & & 8. 148 & 25847.6 & 299481. 0 & 49. 9823 \\
\hline 2 & 2 & & 8. 882 & 23963. 6 & 299693.4 & 50. 0177 \\
\hline Total & & & & 49811.2 & 599174.4 & 100.0000 \\
\hline
\end{tabular}

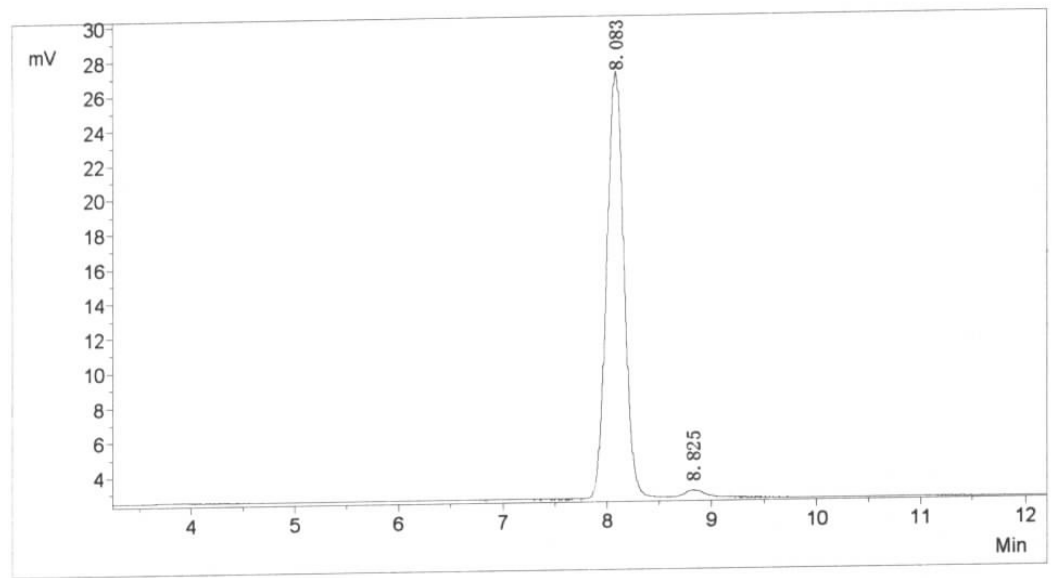

\begin{tabular}{|c|c|c|c|c|c|c|}
\hline No. & PeakNo & ID. Name & R. Time & PeakHe ight & PeakArea & Conc \\
\hline 1 & 1 & & 8. 083 & 24639. 1 & 283613.5 & 98. 8594 \\
\hline 2 & 2 & & 8. 825 & 321.0 & 3272.2 & 1. 1406 \\
\hline Tota & & & & 24960.1 & 286885.8 & 100. 0000 \\
\hline
\end{tabular}

The ee's were determined by GC analysis using a Suplco Chiral select 1000 column, $30 \mathrm{~m} \times 0.25 \mathrm{~mm}$. Oven temperature is $110{ }^{\circ} \mathrm{C}$. Absolute configuration was determined by comparison of the sign of the optical rotation with reported value. ${ }^{2}$ 


\section{Gas chromatograms to determine enantiomeric excesses for hydrogenation}

products of enamides

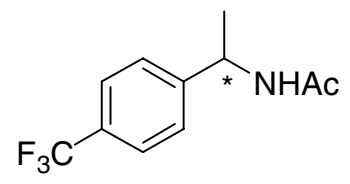

\section{$99.9 \%$ ee (S)}

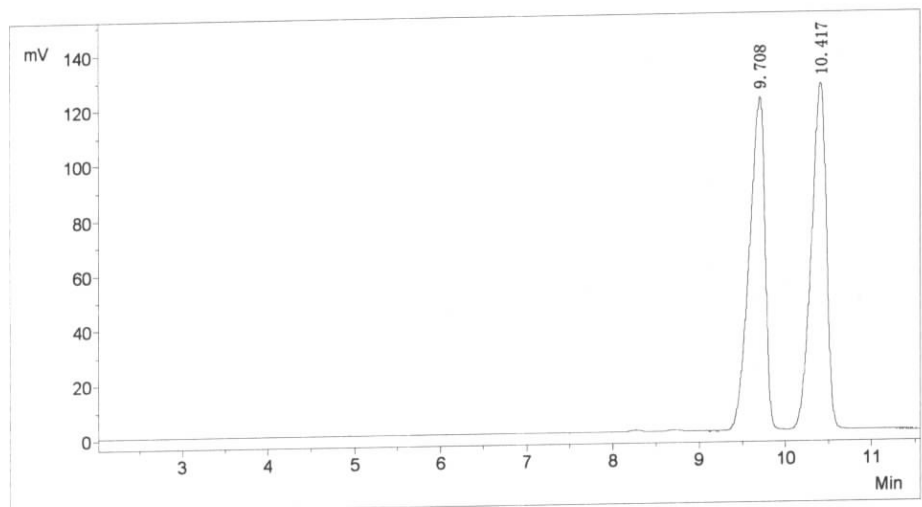

\begin{tabular}{rrrrrr} 
No. PeakNo & ID. Name & R. Time & PeakHeight & PeakArea & \multicolumn{1}{c}{ Conc } \\
\hline 1 & 1 & 9.708 & 121147.8 & 1531439.8 & 50.0049 \\
2 & 2 & 10.417 & 125660.2 & 1531136.8 & 49.9951 \\
\hline Total & & 246808.0 & 3062576.6 & 100.0000
\end{tabular}

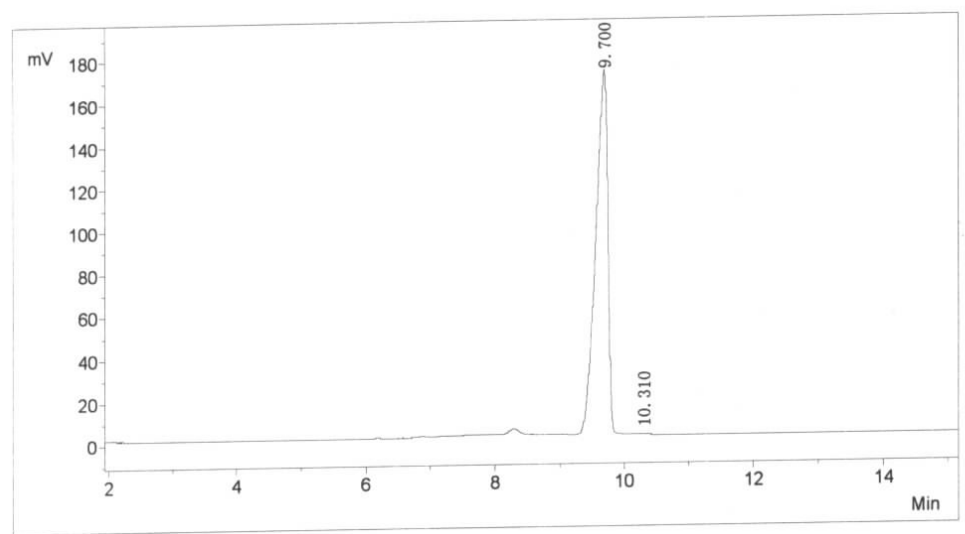

\begin{tabular}{rrrrrr} 
No. PeakNo & ID. Name & R. Time & PeakHeight & PeakArea & \multicolumn{1}{c}{ Conc } \\
\hline 1 & 1 & 9.700 & 171136.1 & 2216830.3 & 99.9668 \\
2 & 2 & 10.310 & 93.8 & 735.2 & 0.0332 \\
\hline Total & & 171230.0 & 2217565.5 & 100.0000
\end{tabular}

The ee's were determined by GC analysis using a Supelco Chiral select 1000 column, $30 \mathrm{~m} \times 0.25 \mathrm{~mm}$. Oven temperature is $150{ }^{\circ} \mathrm{C}$. Absolute configuration was determined by comparison of the sign of the optical rotation with reported value. ${ }^{3}$ 

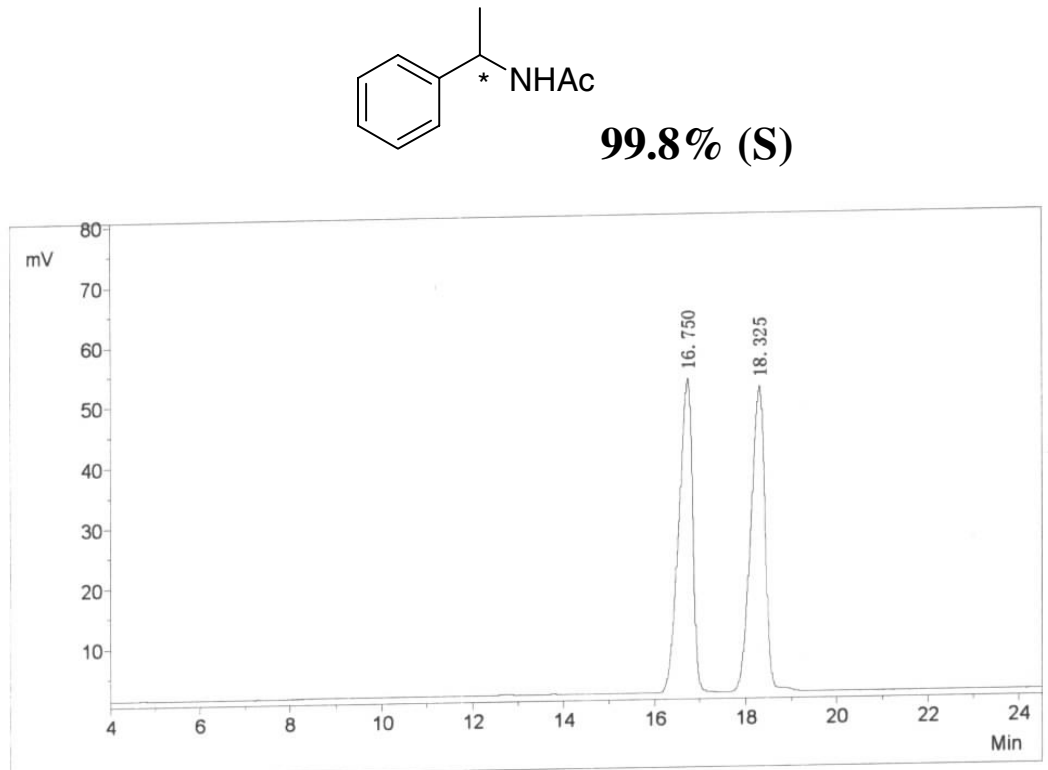

\begin{tabular}{|c|c|c|c|c|c|c|}
\hline No. & PeakNo & ID. Name & R. Time & PeakHe ight & PeakArea & Conc \\
\hline 1 & 1 & & 16.750 & 51782.8 & 1088866. 1 & 49. 8791 \\
\hline 2 & 2 & & 18. 325 & 50357.4 & 1094144.6 & 50. 1209 \\
\hline Tota & & & & 102140. 2 & 2183010.7 & 100.0000 \\
\hline
\end{tabular}

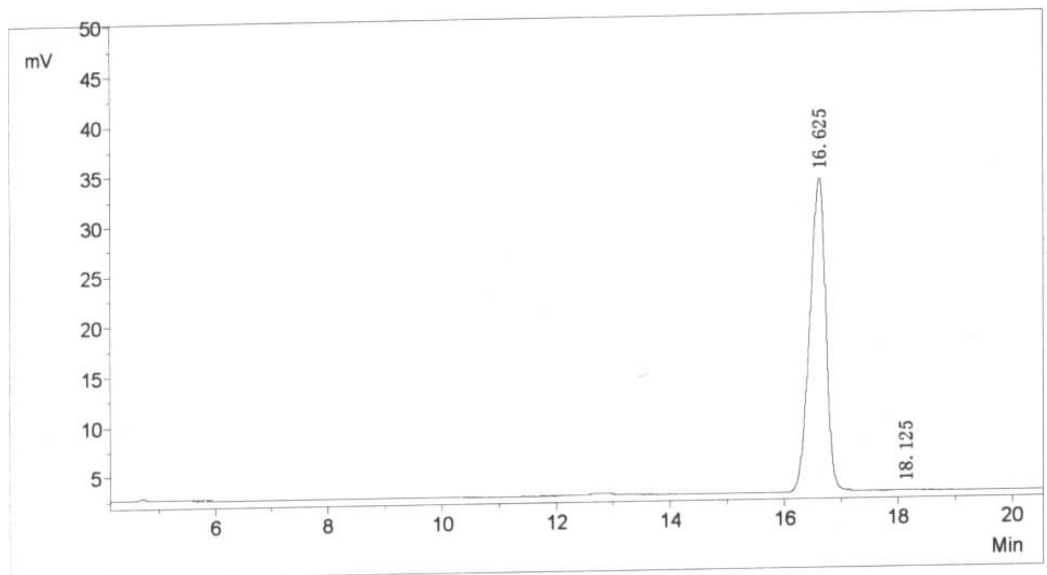

\begin{tabular}{rrrrrr} 
No. PeakNo & ID. Name & R. Time & PeakHeight & PeakArea & \multicolumn{1}{c}{ Conc } \\
\hline 1 & 1 & 16.625 & 31308.8 & 612241.8 & 99.9229 \\
2 & 2 & 18.125 & 38.0 & 472.5 & 0.0771 \\
\hline Total & & 31346.8 & 612714.3 & 100.0000
\end{tabular}

The ee's were determined by GC analysis using a Supelco Chiral select 1000 column, $30 \mathrm{~m} \times 0.25 \mathrm{~mm}$. Oven temperature is $130{ }^{\circ} \mathrm{C}$. Absolute configuration was determined by comparison of the sign of the optical rotation with reported value. ${ }^{3}$ 


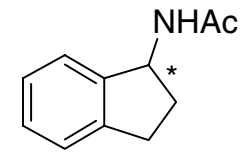

\section{$96.0 \%$ ee $(\mathbf{S})$}

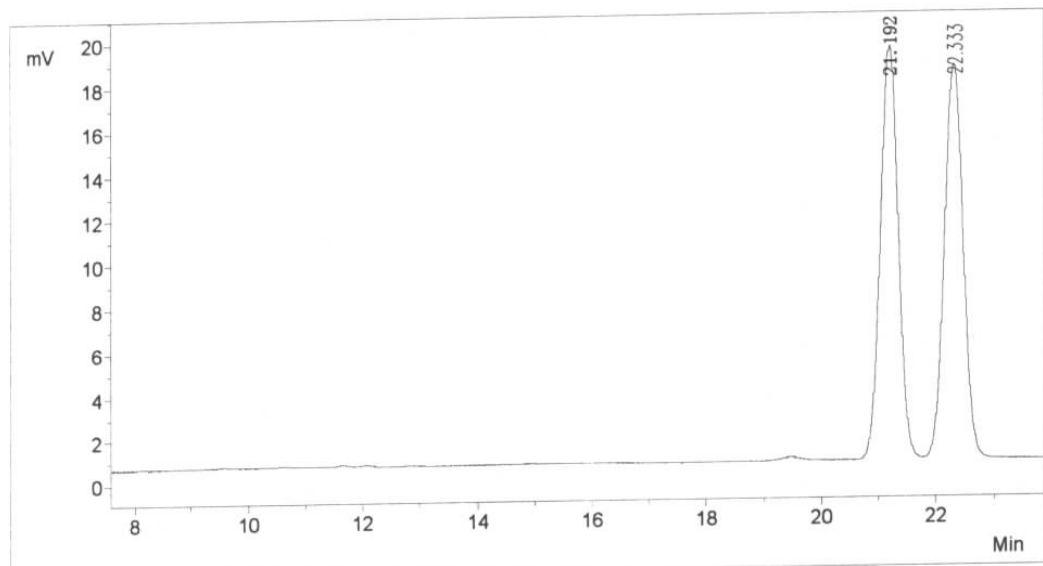

\begin{tabular}{|c|c|c|c|c|c|c|}
\hline No. & PeakNo & ID. Name & R.Time & PeakHeight & PeakArea & Conc \\
\hline $\begin{array}{l}1 \\
2\end{array}$ & $\begin{array}{l}1 \\
2\end{array}$ & & $\begin{array}{l}21.192 \\
22333\end{array}$ & 18702.0 & $\begin{array}{l}404951.2 \\
403739.6\end{array}$ & $\begin{array}{l}50.0749 \\
49.9251\end{array}$ \\
\hline 2 & 2 & & 22.555 & $1 / 7 / 8.9$ & & \\
\hline Total & & & & 36480.9 & 808690.8 & 100.0000 \\
\hline
\end{tabular}

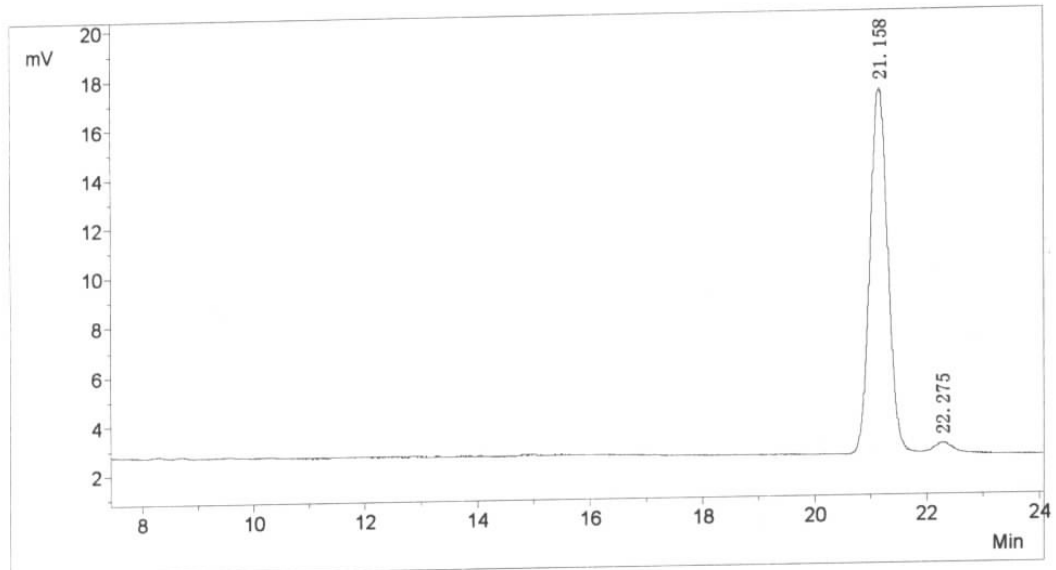

\begin{tabular}{|c|c|c|c|c|c|c|}
\hline No. & PeakNo & ID. Name & R. Time & PeakHeight & PeakArea & Conc \\
\hline 1 & 1 & & 21. 158 & 14736.4 & 324997.0 & 97. 9945 \\
\hline 2 & 2 & & 22. 275 & 331.2 & 6651.3 & 2. 0055 \\
\hline Tota & & & & 15067.6 & 331648. 3 & 100.0000 \\
\hline
\end{tabular}

The ee's were determined by GC analysis using a Supelco Chiral select 1000 column, $30 \mathrm{~m} \times 0.25 \mathrm{~mm}$. Oven temperature is $150{ }^{\circ} \mathrm{C}$. Absolute configuration was determined by comparison of the sign of the optical rotation with reported value. ${ }^{\text {lc }}$ 


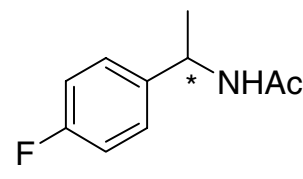

$99.9 \%$ ee $(\mathbf{S})$

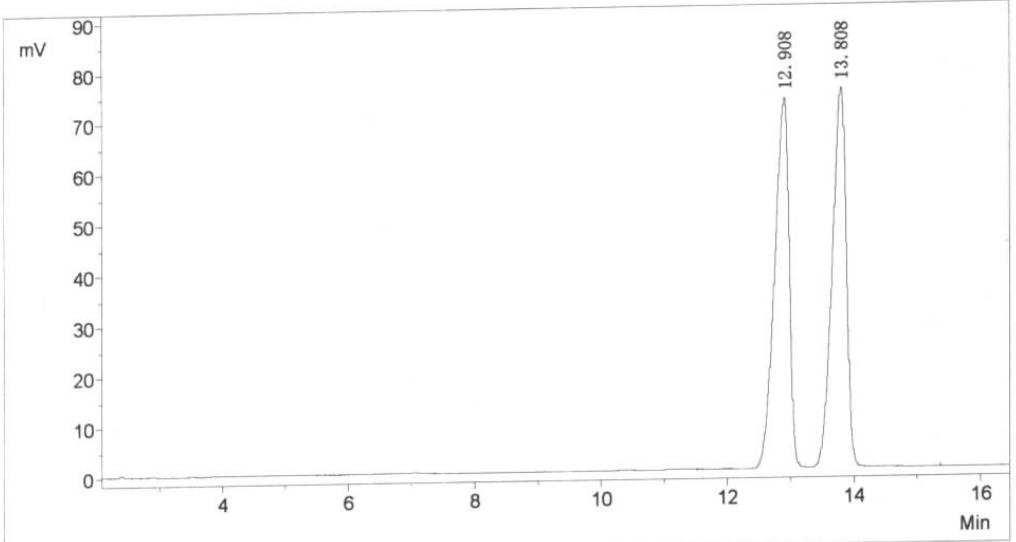

\begin{tabular}{|c|c|c|c|c|c|c|}
\hline No. & PeakNo & ID. Name & R. Time & PeakHeight & PeakArea & Conc \\
\hline 1 & 1 & & 12. 908 & 73039.0 & 1162483.5 & 50.0218 \\
\hline 2 & 2 & & 13. 808 & 74755. 0 & 1161469.0 & 49. 9782 \\
\hline Tota & & & & 147793.9 & 2323952.5 & 100.0000 \\
\hline
\end{tabular}

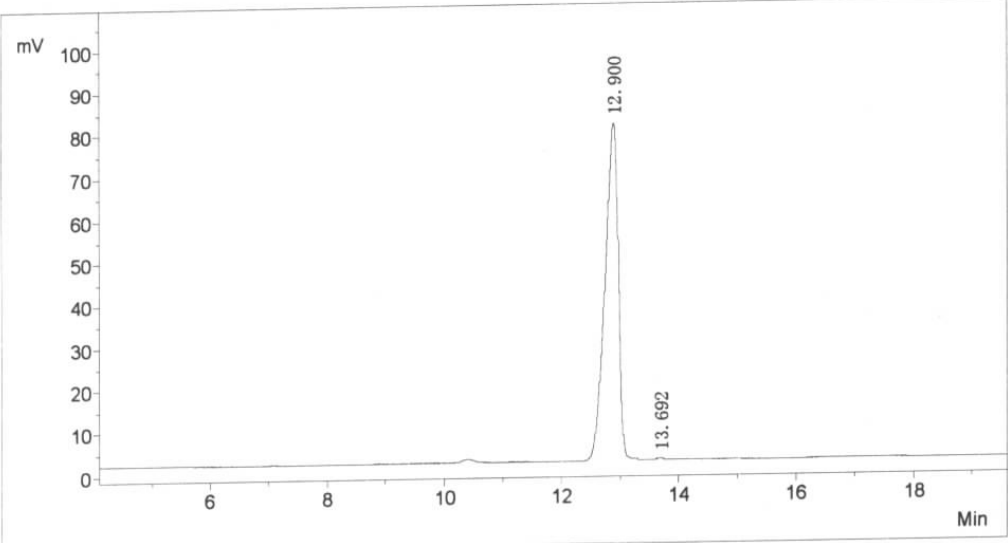

\begin{tabular}{|c|c|c|c|c|c|c|}
\hline No. & PeakNo & ID. Name & R. Time & PeakHe ight & PeakArea & Conc \\
\hline 1 & 1 & & 12. 900 & 79022.4 & 1241855.7 & 99. 9464 \\
\hline 2 & 2 & & 13. 692 & 76. 2 & 666.1 & 0. 0536 \\
\hline Tota & & & & 79098. 7 & 1242521.8 & 100.0000 \\
\hline
\end{tabular}

The ee's were determined by GC analysis using a Supelco Chiral select 1000 column, $30 \mathrm{~m} \times 0.25 \mathrm{~mm}$. Oven temperature is $140{ }^{\circ} \mathrm{C}$. Absolute configuration was determined by comparison of the sign of the optical rotation with reported value. ${ }^{3}$ 
<smiles>COc1ccc(C(C)=NC=NC(C)(C)C)cc1</smiles>

$99.4 \%$ ee (S)

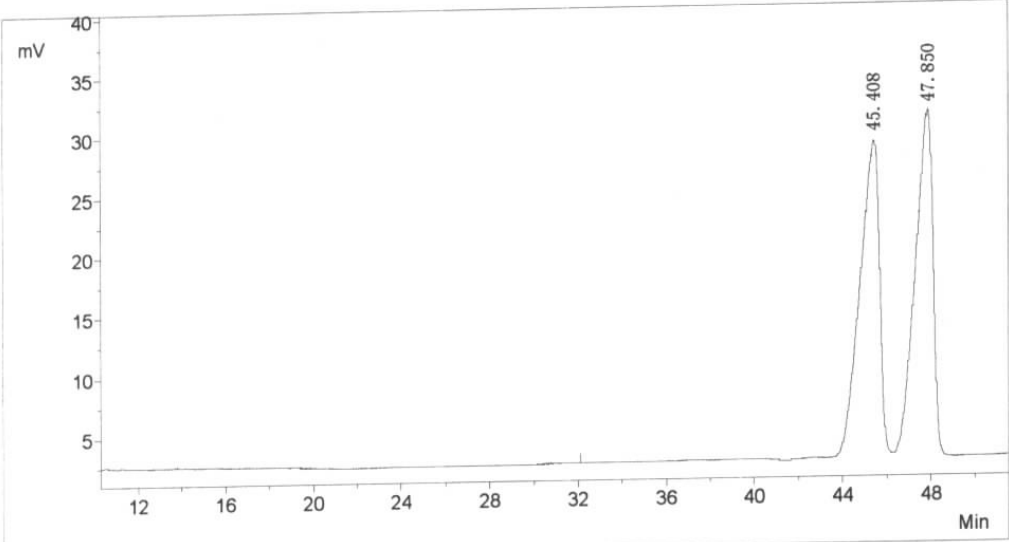

\begin{tabular}{ccrrrc} 
No. PeakNo & ID. Name & R. Time & PeakHeight & PeakArea & \multicolumn{1}{c}{ Conc } \\
\hline 1 & 1 & 45.408 & 26139.1 & 1595895.3 & 50.0633 \\
2 & 2 & 47.850 & 28605.1 & 1591859.5 & 49.9367 \\
\hline Total & & 54744.1 & 3187754.7 & 100.0000
\end{tabular}

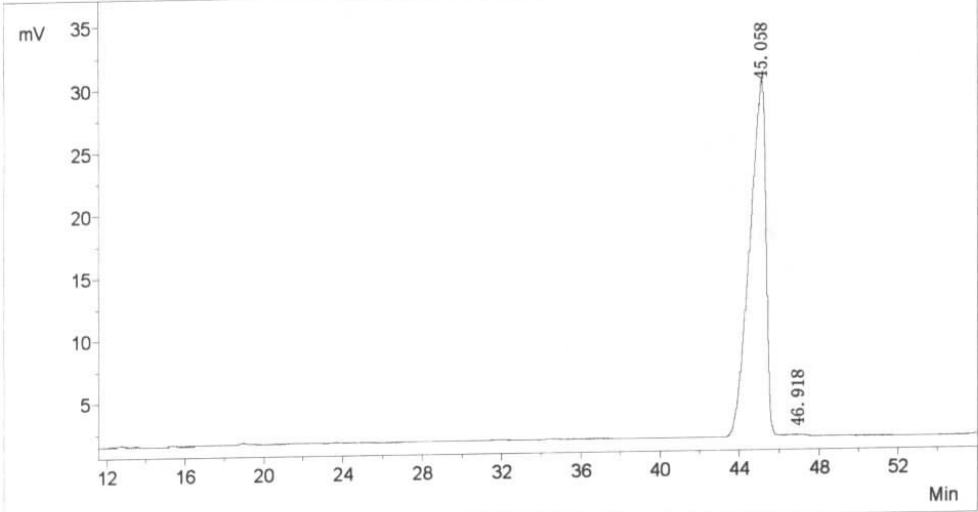

\begin{tabular}{|c|c|c|c|c|c|c|}
\hline No. & PeakNo & ID. Name & R. Time & PeakHeight & PeakArea & Conc \\
\hline 1 & 1 & & 45. 058 & 27676.5 & 1600975.0 & 99. 7256 \\
\hline 2 & 2 & & 46. 918 & 99.6 & 4404.5 & 0. 2744 \\
\hline Tota & & & & 27776.1 & 1605379.5 & 100.0000 \\
\hline
\end{tabular}

The ee's were determined by GC analysis using a Supelco Chiral select 1000 column, $30 \mathrm{~m} \times 0.25 \mathrm{~mm}$. Oven temperature is $140{ }^{\circ} \mathrm{C}$. Absolute configuration was determined by comparison of the sign of the optical rotation with reported value. ${ }^{3}$ 


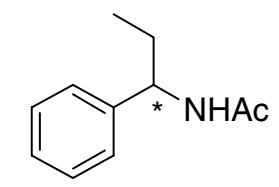

$99.2 \%$ ee (S)

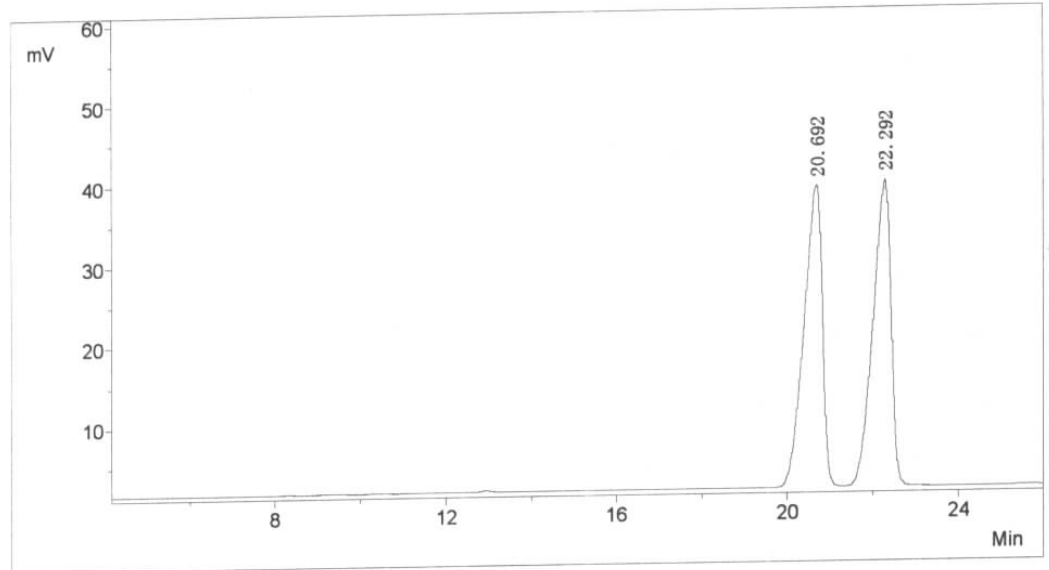

\begin{tabular}{cccrcc} 
No. PeakNo & ID. Name & R. Time & PeakHeight & PeakArea & \multicolumn{1}{c}{ Conc } \\
\hline 1 & 1 & 20.692 & 37405.7 & 1109405.7 & 49.9869 \\
2 & 2 & 22.292 & 37970.5 & 1109988.8 & 50.0131 \\
\hline Total & & 75376.2 & 2219394.5 & 100.0000
\end{tabular}

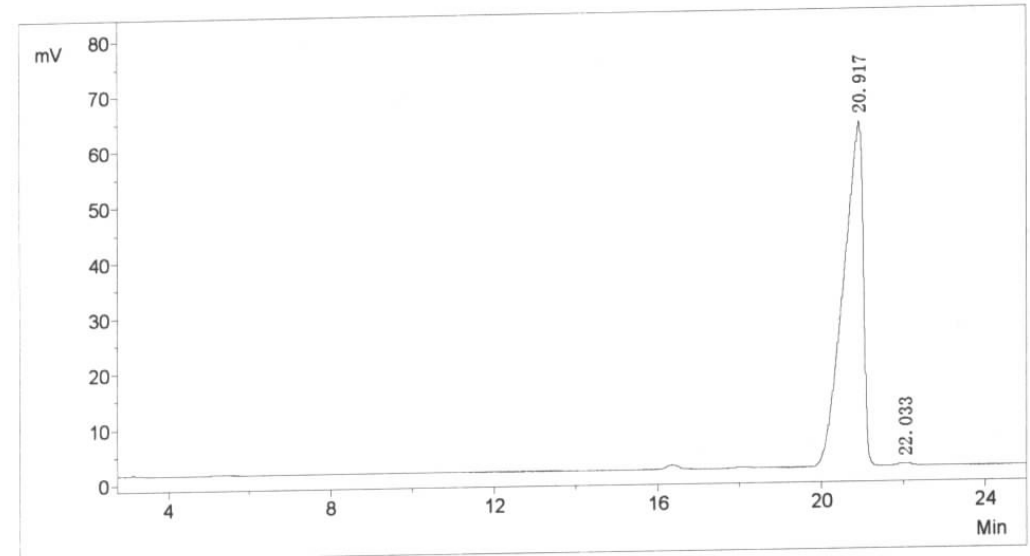

\begin{tabular}{|c|c|c|c|c|c|c|}
\hline No. & PeakNo & ID. Name & R. T ime & PeakHe ight & PeakArea & Conc \\
\hline 1 & 1 & & 20.917 & 61900.0 & 2064421.4 & 99.6108 \\
\hline 2 & 2 & & 22. 033 & 355.9 & 8065. 1 & 0. 3892 \\
\hline Tota & & & & 62255. 9 & 2072486.5 & 100.0000 \\
\hline
\end{tabular}

The ee's were determined by GC analysis using a Supelco Chiral select 1000 column, $30 \mathrm{~m} \times 0.25 \mathrm{~mm}$. Oven temperature is $130{ }^{\circ} \mathrm{C}$. Absolute configuration was determined by comparison of the sign of the optical rotation with reported value. ${ }^{3}$ 
<smiles>CC(=O)N=C(C)c1ccc2ccccc2c1</smiles>

$99.5 \%$ ee (S)

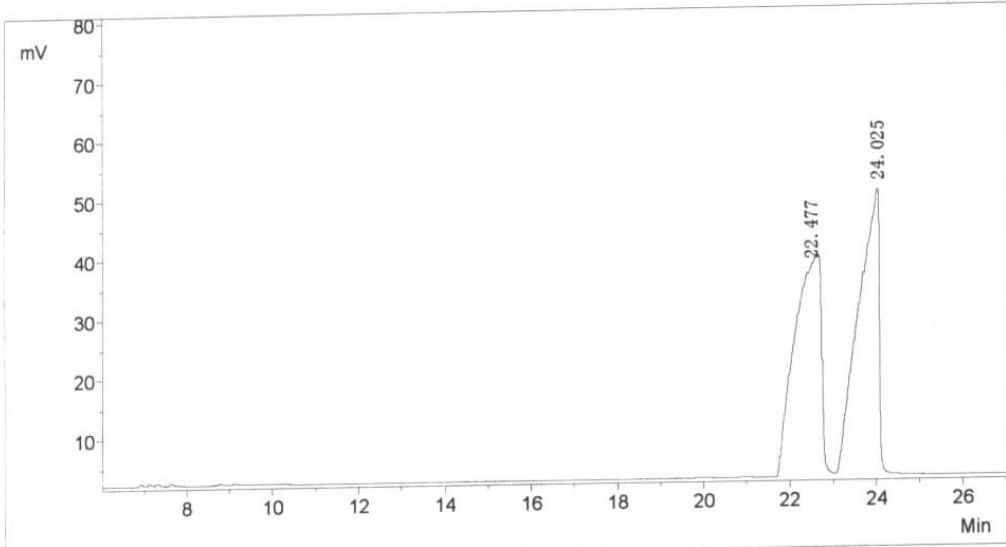

\begin{tabular}{rrrrrc} 
No. PeakNo & ID. Name & R. Time & PeakHeight & PeakArea & \multicolumn{1}{c}{ Conc } \\
\hline 1 & 1 & 22.477 & 34670.7 & 1557429.8 & 49.9596 \\
2 & 2 & 24.025 & 47787.9 & 1559950.7 & 50.0404 \\
\hline Total & & 82458.6 & 3117380.5 & 100.0000
\end{tabular}

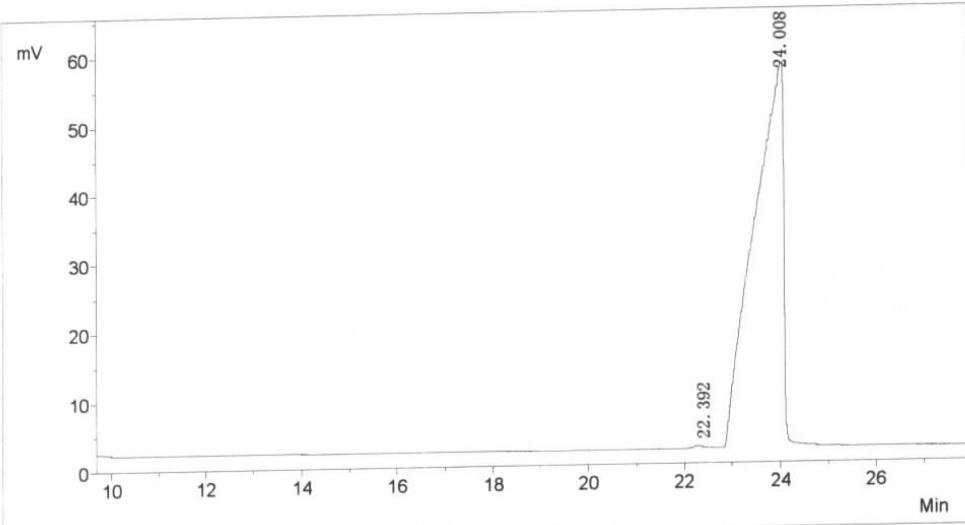

\begin{tabular}{rrrrrr} 
No. PeakNo & ID. Name & R. Time & PeakHeight & \multicolumn{1}{c}{ PeakArea } & \multicolumn{1}{c}{ Conc } \\
\hline 1 & 1 & 22.392 & 238.1 & 6062.4 & 0.2554 \\
2 & 2 & 24.008 & 55470.0 & 2367358.8 & 99.7446 \\
\hline Total & & 55708.1 & 2373421.2 & 100.0000
\end{tabular}

The ee's were determined by GC analysis using a Chrompack chiral fused silica $25 \mathrm{~m} \mathrm{x}$ $0.25 \mathrm{~mm}$ chirasil-L-Val column. Oven temperature is $170{ }^{\circ} \mathrm{C}$. Absolute configuration was determined by comparison of the sign of the optical rotation with reported value. ${ }^{3}$ 


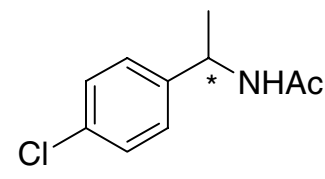

$99.7 \%$ ee $(\mathbf{S})$

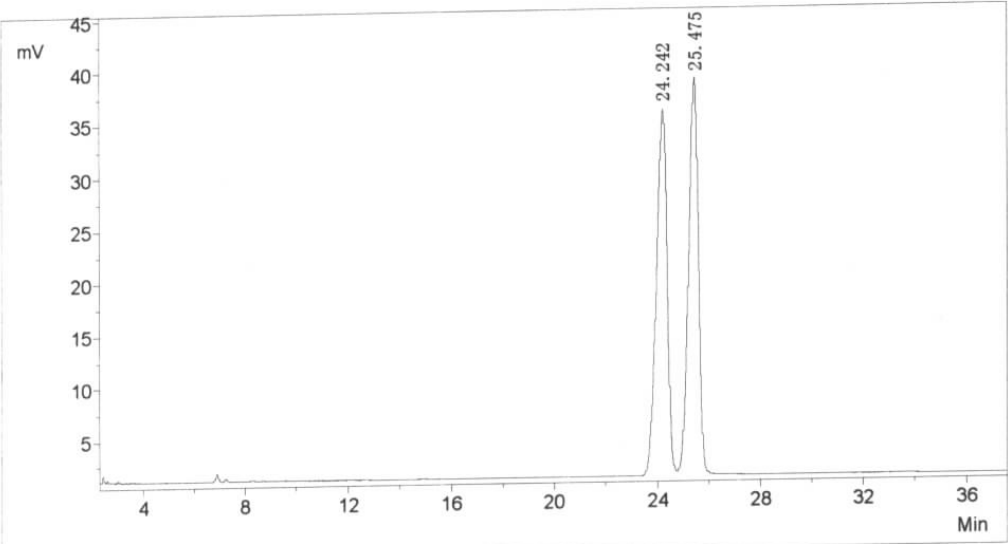

\begin{tabular}{rrrrrr} 
No. PeakNo & ID. Name & R. Time & PeakHeight & PeakArea & \multicolumn{1}{c}{ Conc } \\
\hline 1 & 1 & 24.242 & 34615.9 & 999145.1 & 50.0058 \\
2 & 2 & 25.475 & 37626.3 & 998913.5 & 49.9942 \\
\hline Total & & & 72242.3 & 1998058.5 & 100.0000
\end{tabular}

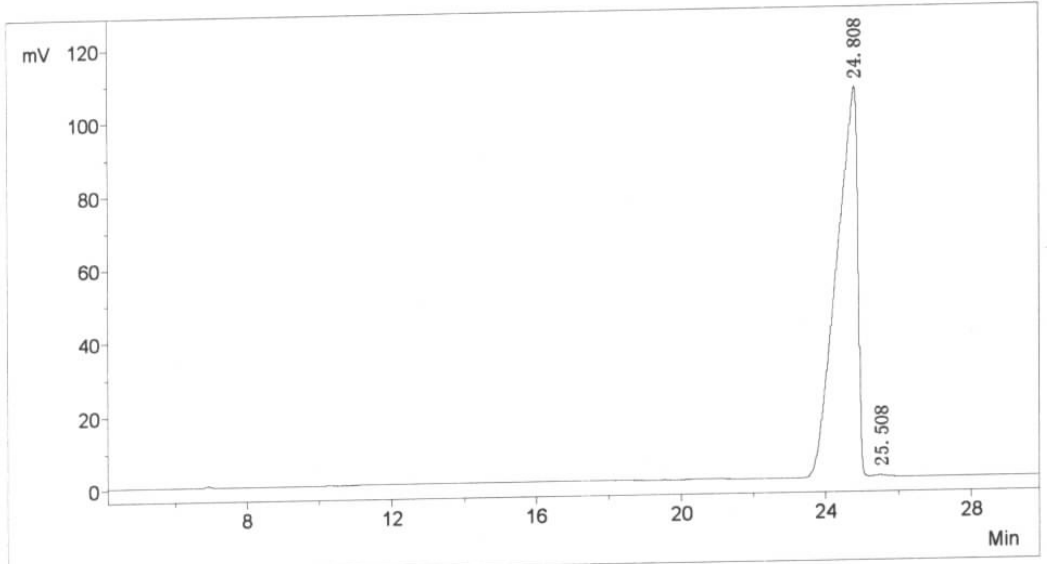

\begin{tabular}{|c|c|c|c|c|c|c|}
\hline No. & PeakNo & ID. Name & R. Time & PeakHe ight & PeakArea & Conc \\
\hline 1 & 1 & & 24.808 & 105928. 2 & 4243092.4 & 99. 8492 \\
\hline 2 & 2 & & 25.508 & 363.8 & 6406.9 & 0.1508 \\
\hline Total & & & & 106292.0 & 4249499. 3 & 100. 0000 \\
\hline
\end{tabular}

The ee's were determined by GC analysis using a Supleco Chiral select 1000 column, $30 \mathrm{~m} \times 0.25 \mathrm{~mm}$. Oven temperature is $150{ }^{\circ} \mathrm{C}$. Absolute configuration was determined by comparison of the sign of the optical rotation with reported value. ${ }^{3}$ 


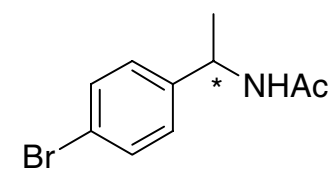

\section{$99.9 \%$ ee $(\mathbf{S})$}

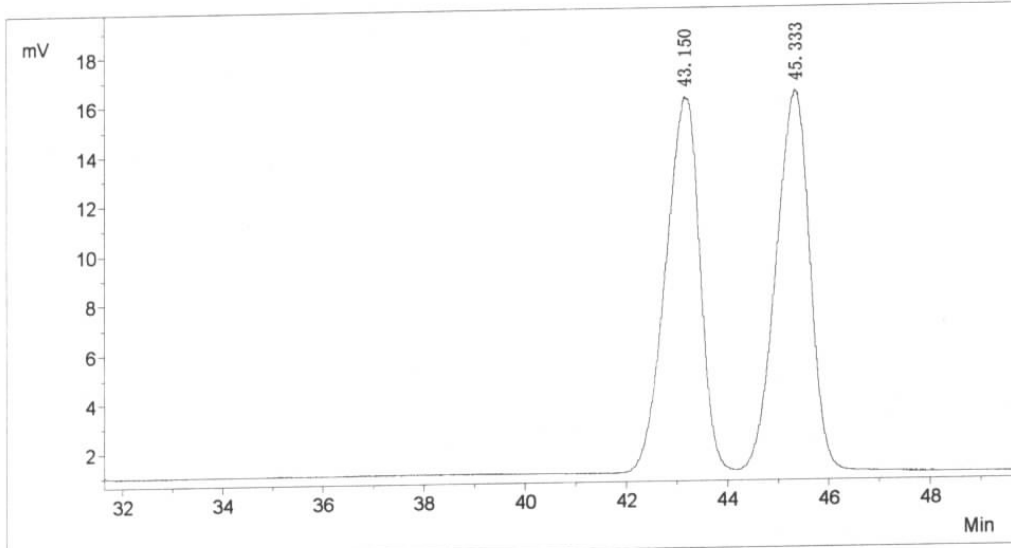

\begin{tabular}{ccrrrr} 
No. PeakNo & ID. Name & R. Time & PeakHeight & PeakArea & \multicolumn{1}{c}{ Conc } \\
\hline 1 & 1 & 43.150 & 15099.1 & 702170.3 & 50.0181 \\
2 & 2 & 45.333 & 15299.0 & 701661.5 & 49.9819 \\
\hline Total & & & 30398.2 & 1403831.8 & 100.0000
\end{tabular}

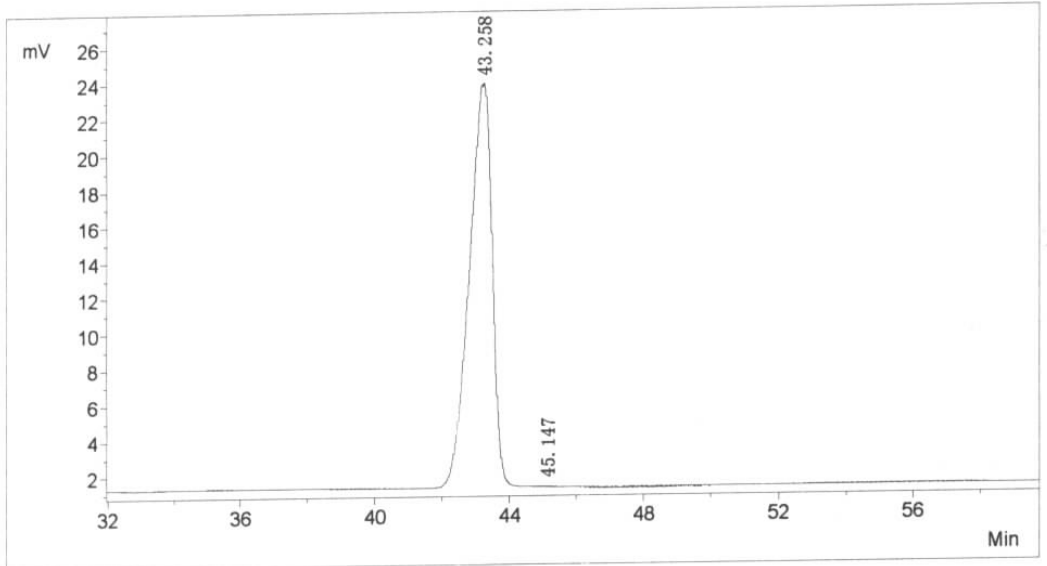

\begin{tabular}{rrrrrr} 
No. PeakNo & ID. Name & R. Time & PeakHeight & PeakArea & \multicolumn{1}{c}{ Conc } \\
\hline 1 & 1 & 43.258 & 22639.2 & 1067953.0 & 99.9712 \\
2 & 2 & 45.147 & 12.5 & 307.8 & 0.0288 \\
\hline Total & & 22651.6 & 1068260.8 & 100.0000
\end{tabular}

The ee's were determined by GC analysis using a Supelco Chiral select 1000 column, $30 \mathrm{~m} \times 0.25 \mathrm{~mm}$. Oven temperature is $150{ }^{\circ} \mathrm{C}$. Absolute configuration was determined by comparison of the sign of the optical rotation with reported value. ${ }^{3}$ 


\section{Gas chromatograms to determine enantiomeric excesses for hydrogenation}

products of $\beta$-(acylamino)acrylates
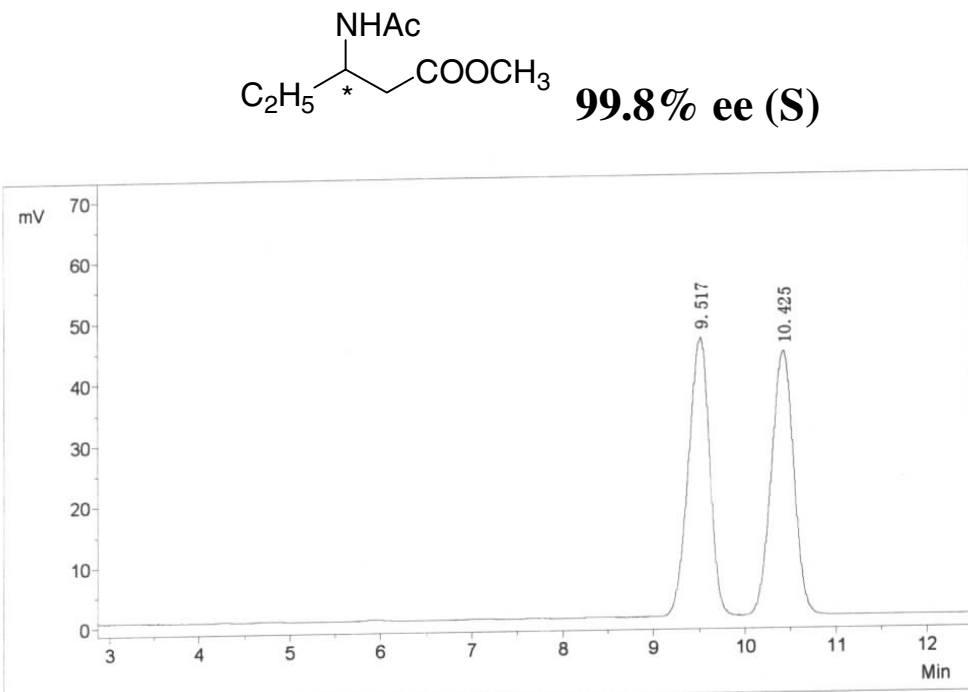

\begin{tabular}{|c|c|c|c|c|c|c|}
\hline No. & PeakNo & ID. Name & R. Time & PeakHeight & PeakArea & Conc \\
\hline 1 & 1 & & 9. 517 & 45580.0 & 741115.5 & 49. 9963 \\
\hline 2 & 2 & & 10.425 & 43138. 3 & 741224. 2 & 50.0037 \\
\hline Tota & & & & 88718.3 & 1482339.7 & 100.0000 \\
\hline
\end{tabular}

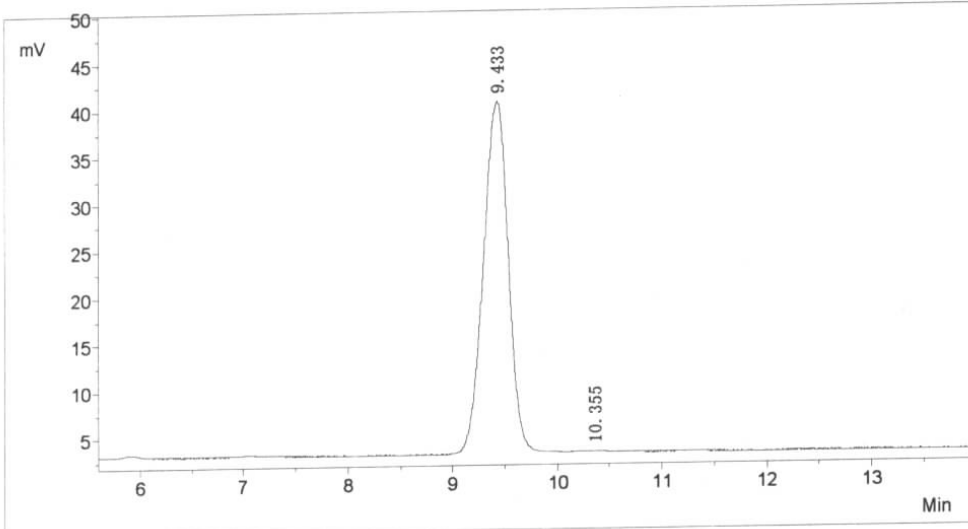

\begin{tabular}{rrrrrr} 
No. PeakNo & ID. Name & R. Time & PeakHeight & PeakArea & \multicolumn{1}{c}{ Conc } \\
\hline 1 & 1 & 9.433 & 37557.8 & 618948.0 & 99.9182 \\
2 & 2 & 10.355 & 55.6 & 506.9 & 0.0818 \\
\hline Total & & 37613.4 & 619454.9 & 100.0000
\end{tabular}

The ee's were determined by GC analysis using a Supelco Chiral select 1000 column, $30 \mathrm{~m} \times 0.25 \mathrm{~mm}$. Oven temperature is $110{ }^{\circ} \mathrm{C}$. Absolute configuration was determined by comparison of the sign of the optical rotation with reported value. ${ }^{\text {dd }}$ 

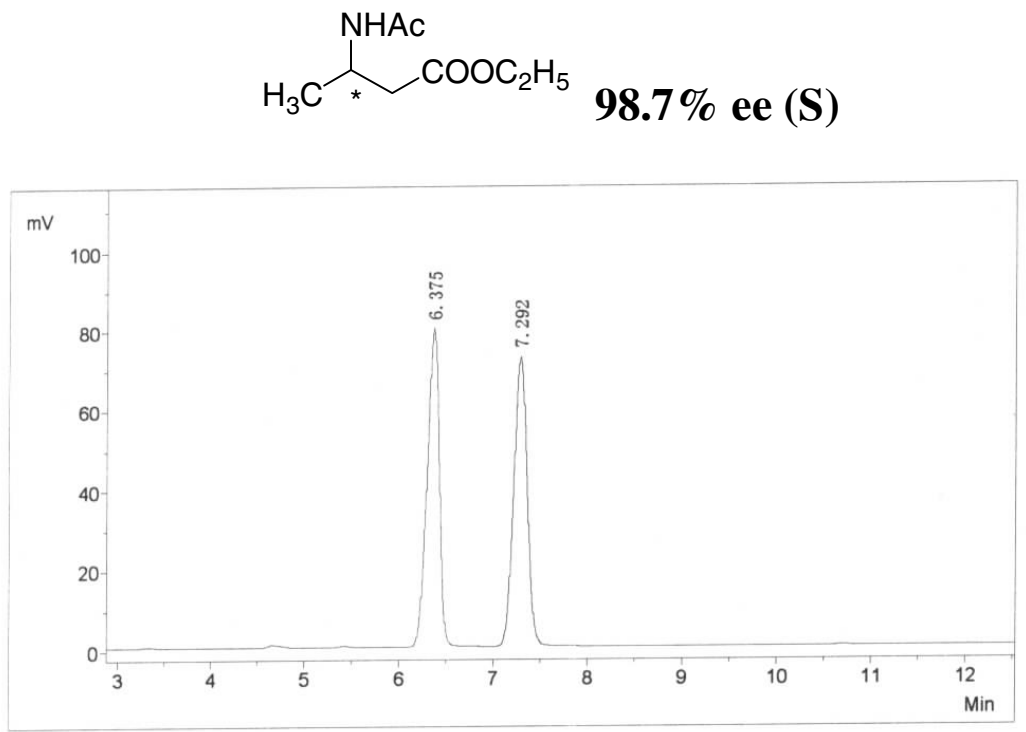

\begin{tabular}{ccrrrr} 
No. PeakNo & ID. Name & R. Time & PeakHeight & \multicolumn{1}{c}{ PeakArea } & \multicolumn{1}{c}{ Conc } \\
\hline 1 & 1 & 6.375 & 79704.9 & 719528.9 & 50.0069 \\
2 & 2 & 7.292 & 72345.8 & 719330.7 & 49.9931 \\
\hline Total & & & 152050.8 & 1438859.6 & 100.0000
\end{tabular}

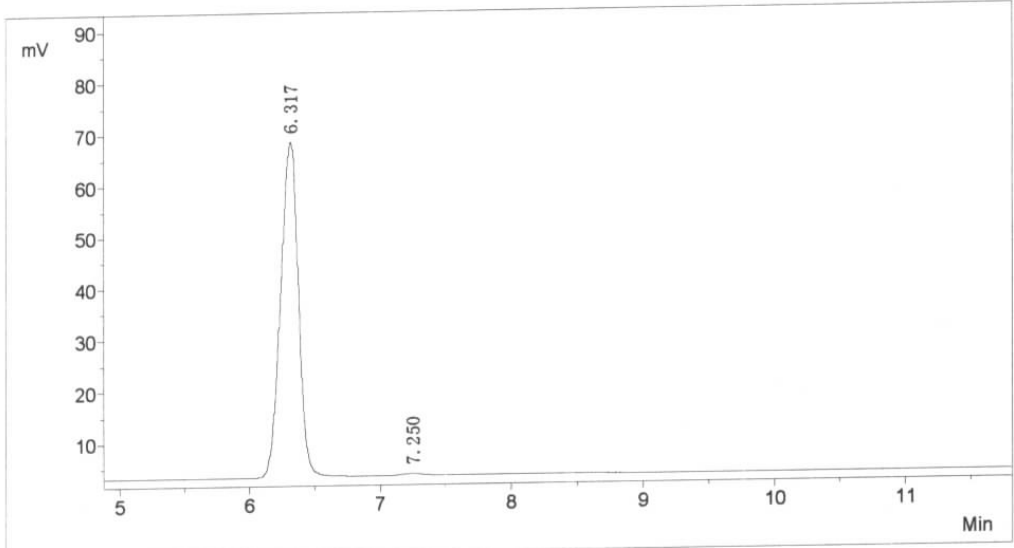

\begin{tabular}{|c|c|c|c|c|c|c|}
\hline No. & PeakNo & ID. Name & R. Time & PeakHe ight & PeakArea & Conc \\
\hline 1 & 1 & & 6. 317 & 65159.8 & 613897.2 & 99. 3672 \\
\hline 2 & 2 & & 7. 250 & 400.5 & 3909.8 & 0.6328 \\
\hline Total & & & & 65560.3 & 617807.0 & 100.0000 \\
\hline
\end{tabular}

The ee's were determined by GC analysis using a Supelco Chiral select 1000 column, $30 \mathrm{~m} \times 0.25 \mathrm{~mm}$. Oven temperature is $130{ }^{\circ} \mathrm{C}$. Absolute configuration was determined by comparison of the sign of the optical rotation with reported value. ${ }^{1 \mathrm{~d}}$ 


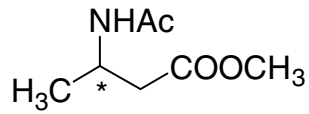

\section{$99.1 \%$ ee $(\mathrm{S})$}

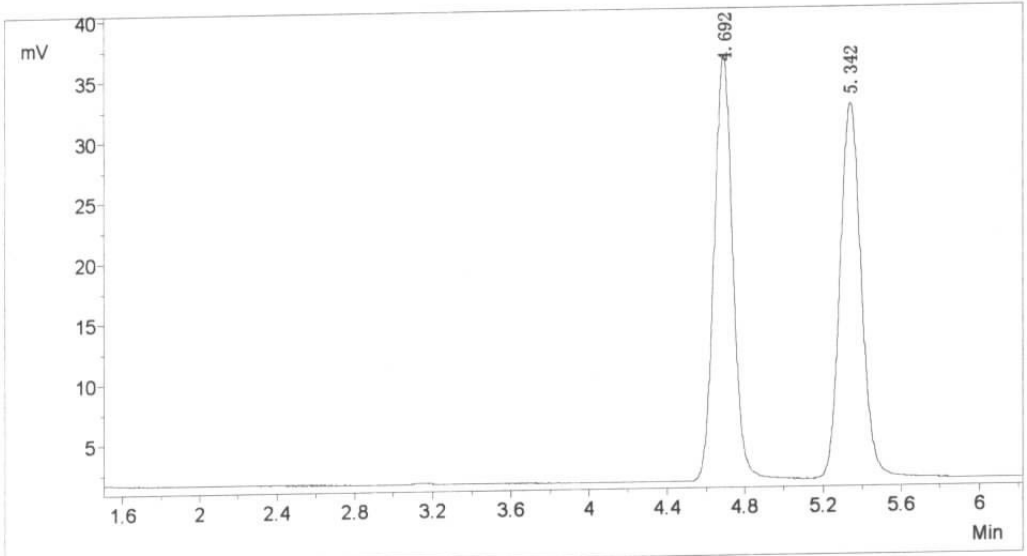

\begin{tabular}{cccrrc} 
No. PeakNo & ID. Name & R. Time & PeakHeight & PeakArea & \multicolumn{1}{c}{ Conc } \\
\hline 1 & 1 & 4.692 & 34963.0 & 233310.9 & 49.9584 \\
2 & 2 & 5.342 & 30921.4 & 233699.7 & 50.0416 \\
\hline Total & & & 65884.4 & 467010.6 & 100.0000
\end{tabular}

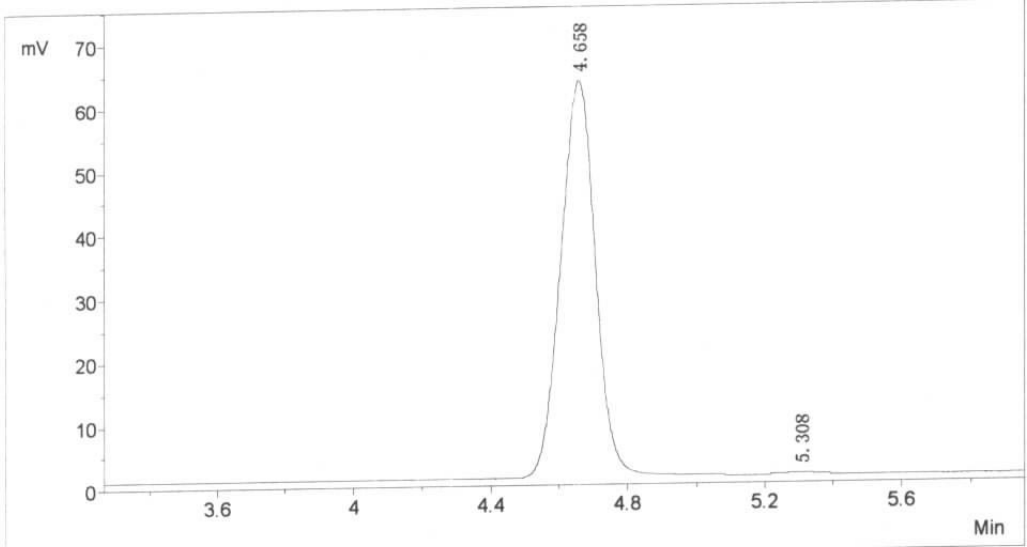

\begin{tabular}{rrrrrr} 
No. PeakNo & ID. Name & R. Time & PeakHeight & \multicolumn{1}{c}{ PeakArea } & \multicolumn{1}{c}{ Conc } \\
\hline 1 & 1 & 4.658 & 62379.9 & 439628.8 & 99.5825 \\
2 & 2 & 5.308 & 259.5 & 1843.2 & 0.4175 \\
\hline Total & & & 62639.3 & 441472.0 & 100.0000
\end{tabular}

The ee's were determined by GC analysis using a Supelco Chiral select 1000 column, $30 \mathrm{~m} \times 0.25 \mathrm{~mm}$. Oven temperature is $130{ }^{\circ} \mathrm{C}$. Absolute configuration was determined by comparison of the sign of the optical rotation with reported value. ${ }^{1 \mathrm{~d}}$ 


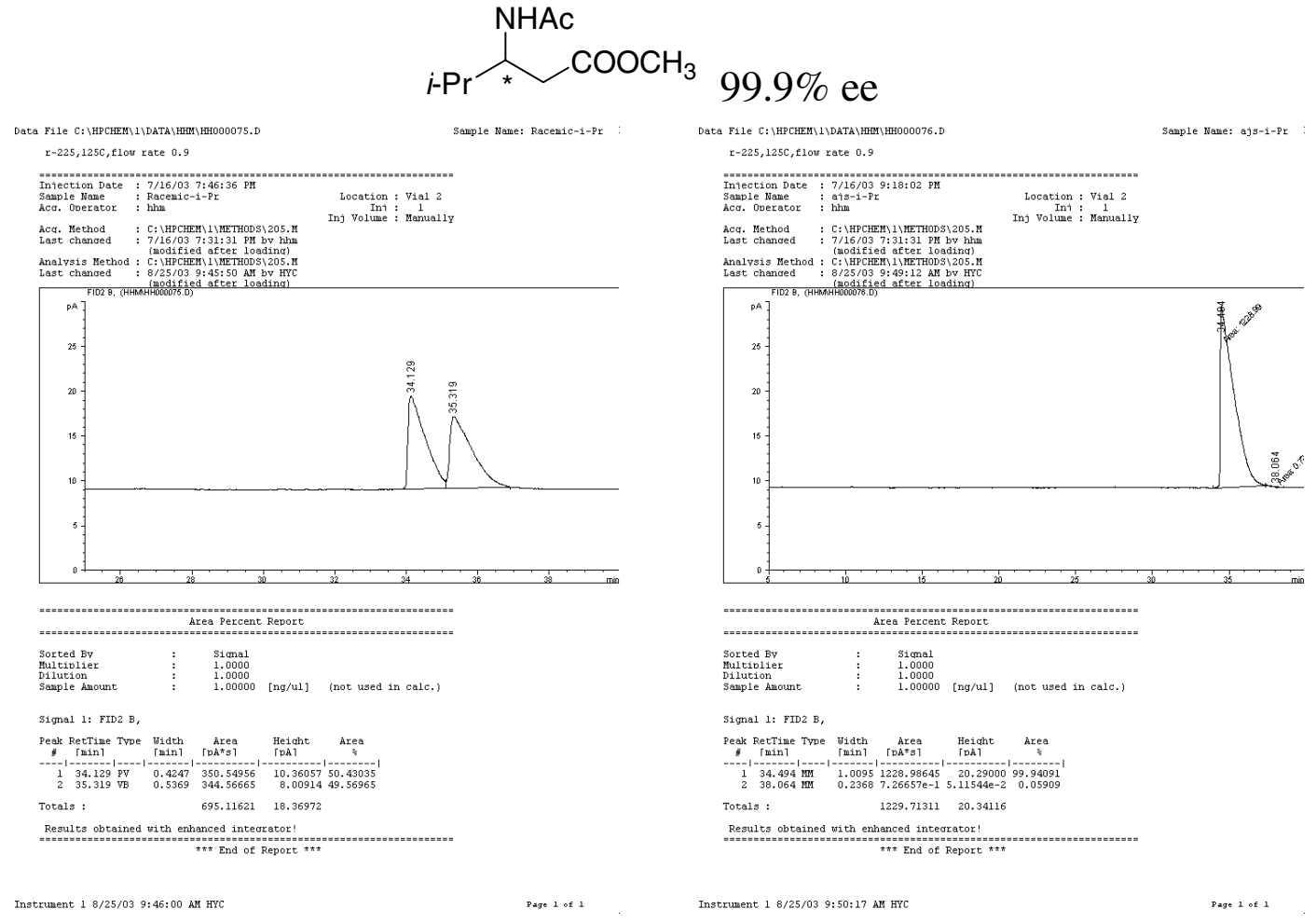

The ee's were determined by GC analysis using a Supelco $\gamma$-DEX 225 column, 30m x $0.25 \mathrm{~mm}$. Oven temperature is $125{ }^{\circ} \mathrm{C}$. Absolute configuration was determined by comparison of the sign of the optical rotation with reported value. ${ }^{1 \mathrm{~d}}$

\section{Gas chromatograms to determine enantiomeric excesses for hydrogenation products of dimethyl itaconate}
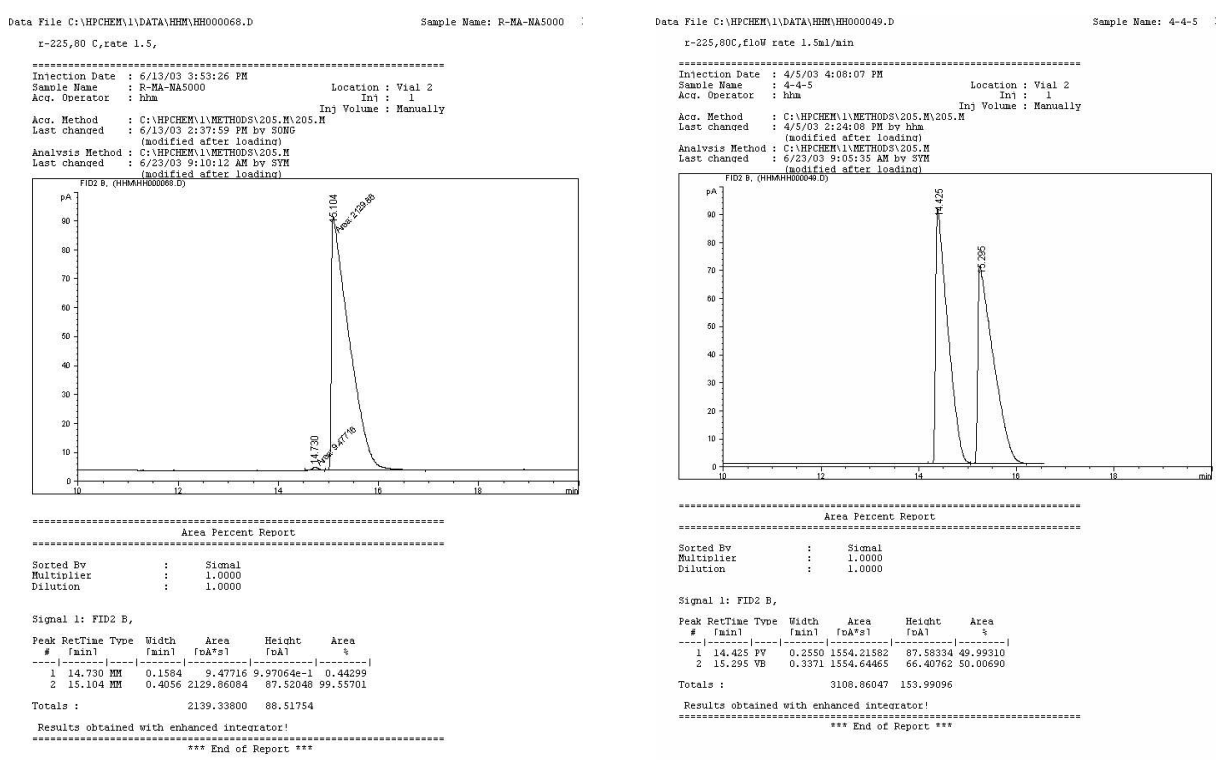

$\mathrm{S} 20$ 


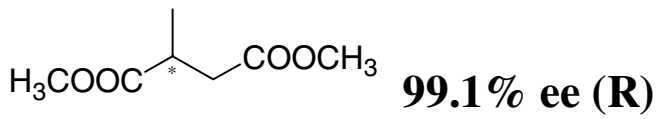

The ee's were determined by GC analysis using a Supelco $\gamma$-DEX 225 column, 30m x $0.25 \mathrm{~mm}$. Oven temperature is $80{ }^{\circ} \mathrm{C}$. Absolute configuration was determined by comparison of the sign of the optical rotation with reported value. ${ }^{4}$

\section{References}

(1) (a) Herbst, R. M.; Shemin, D. Organic Syntheses, Collect. Vol, II, Wiley, New York, N. Y., 1943, p 1; (b) Burk, M. J.; Casy, G.; Johnson, N. B. J. Org. Chem. 1998, 63, 6084; (c) Zhang, Z.; Zhu, G.; Jiang, Q.; Xiao, D.; Zhang, X. J. Org. Chem. 1999, 64, 1774; (d) Zhu, G.; Chen, Z.; Zhang, X. J. Org. Chem. 1999, 64, 6907.

(2) Burk, M. J.; Feaster, J. E.; Nugent, W. A.; Harlow, R. L. J. Am. Chem. Soc. 1993, $115,10125$.

(3) (a) Burk, M. J.; Wang, Y. M.; Lee, J. R. J. Am. Chem. Soc. 1996, 118, 5142; (b) Zhu, G..; Casalnuovo, A. L.; Zhang, X. J. Org. Chem. 1998, 63, 8100.

(4) Berens, U.; Burk, M. J.; Gerlach, A.; Hems, W. Angew. Chem. Int. Ed. Engl. 2000, 39, 1981.

\section{(B) NMR for 3a-k}



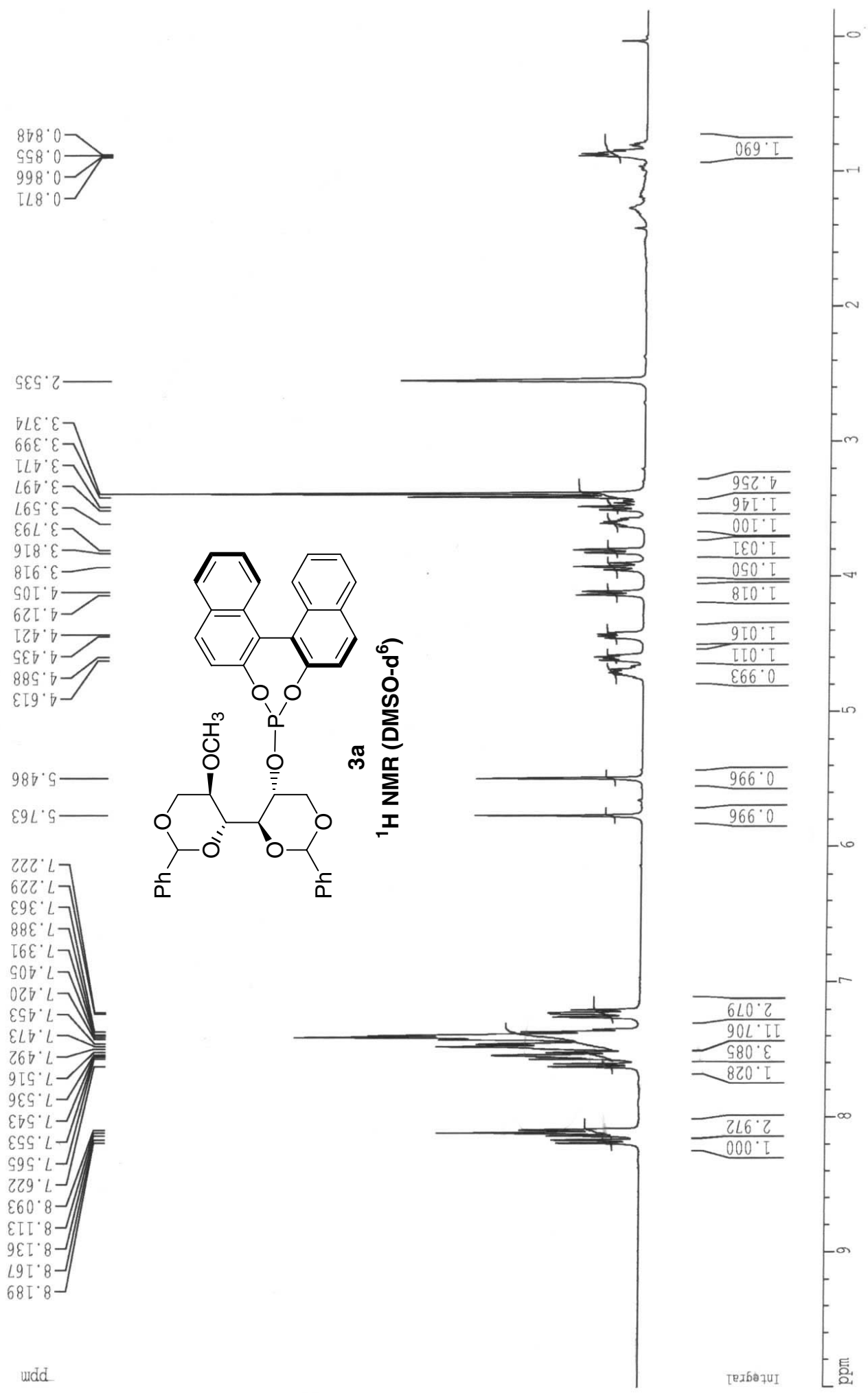

กิ 


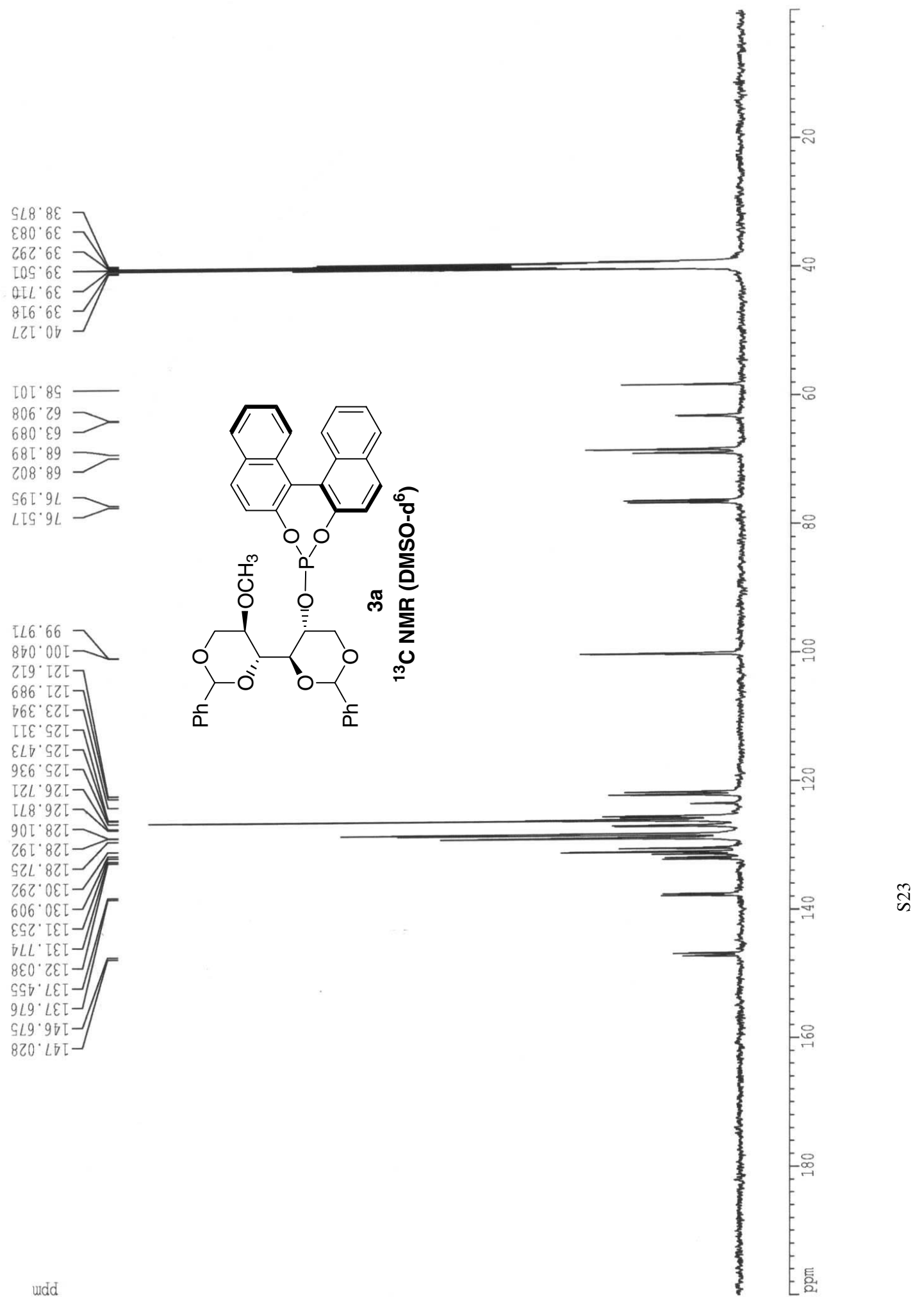




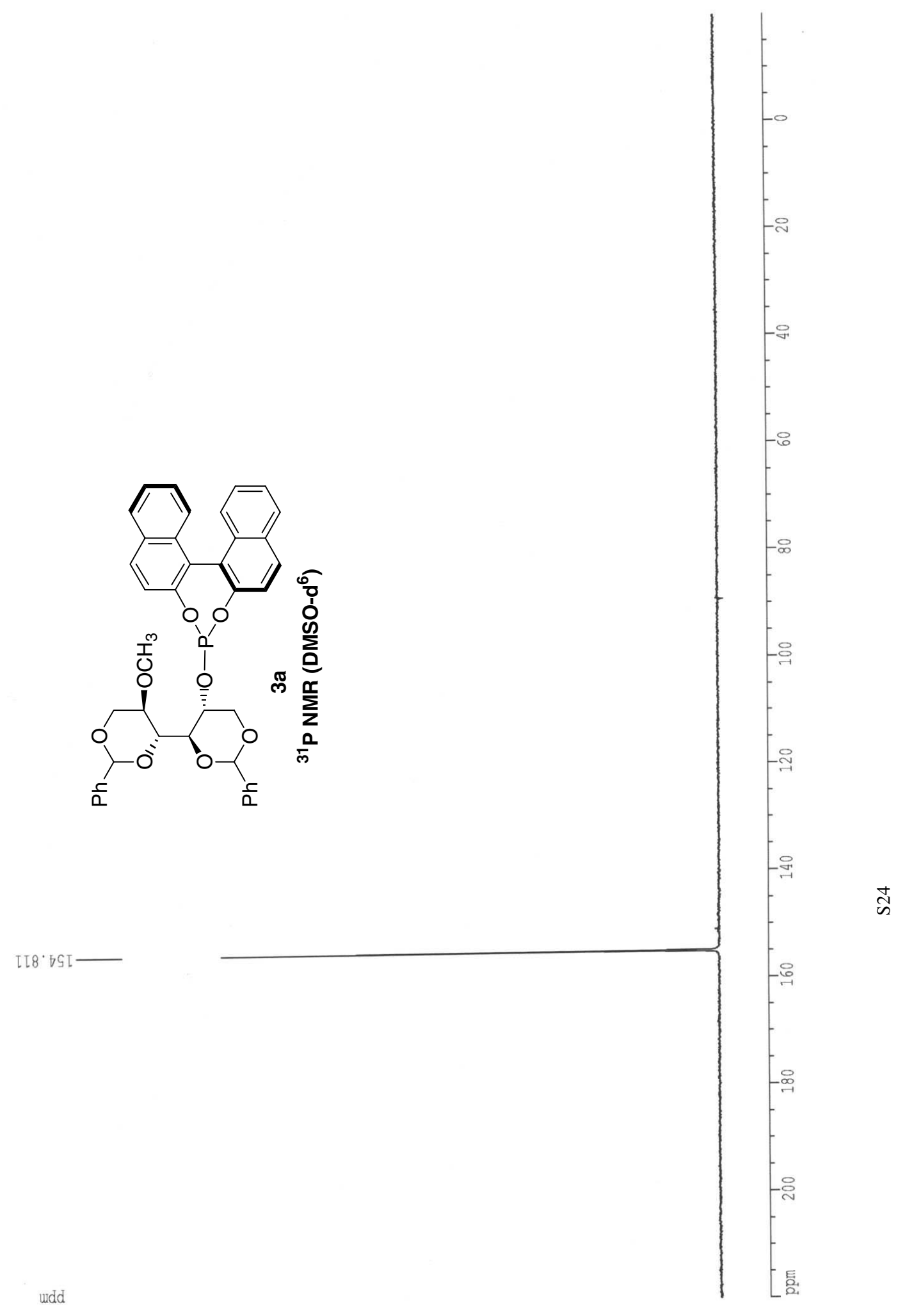




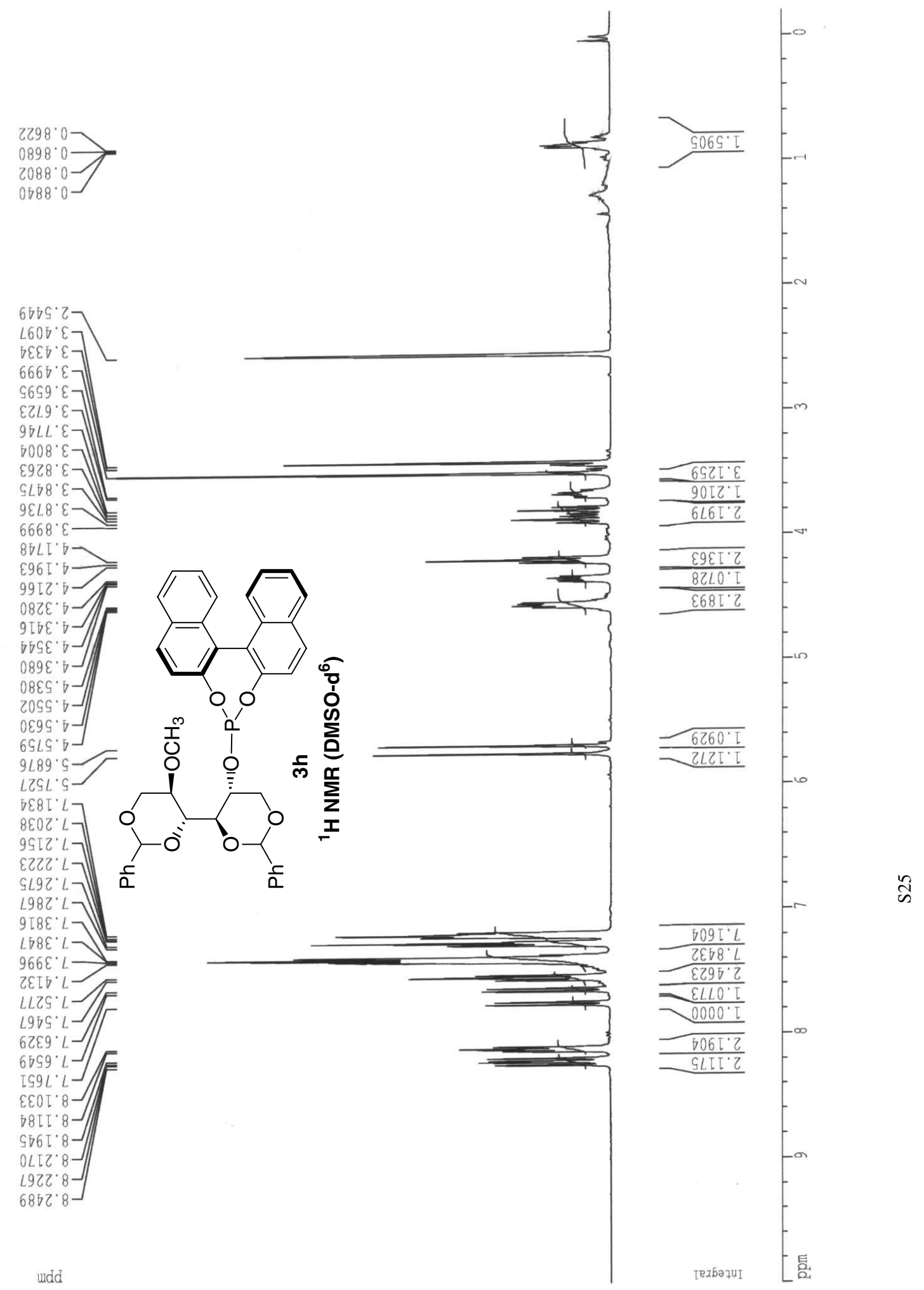




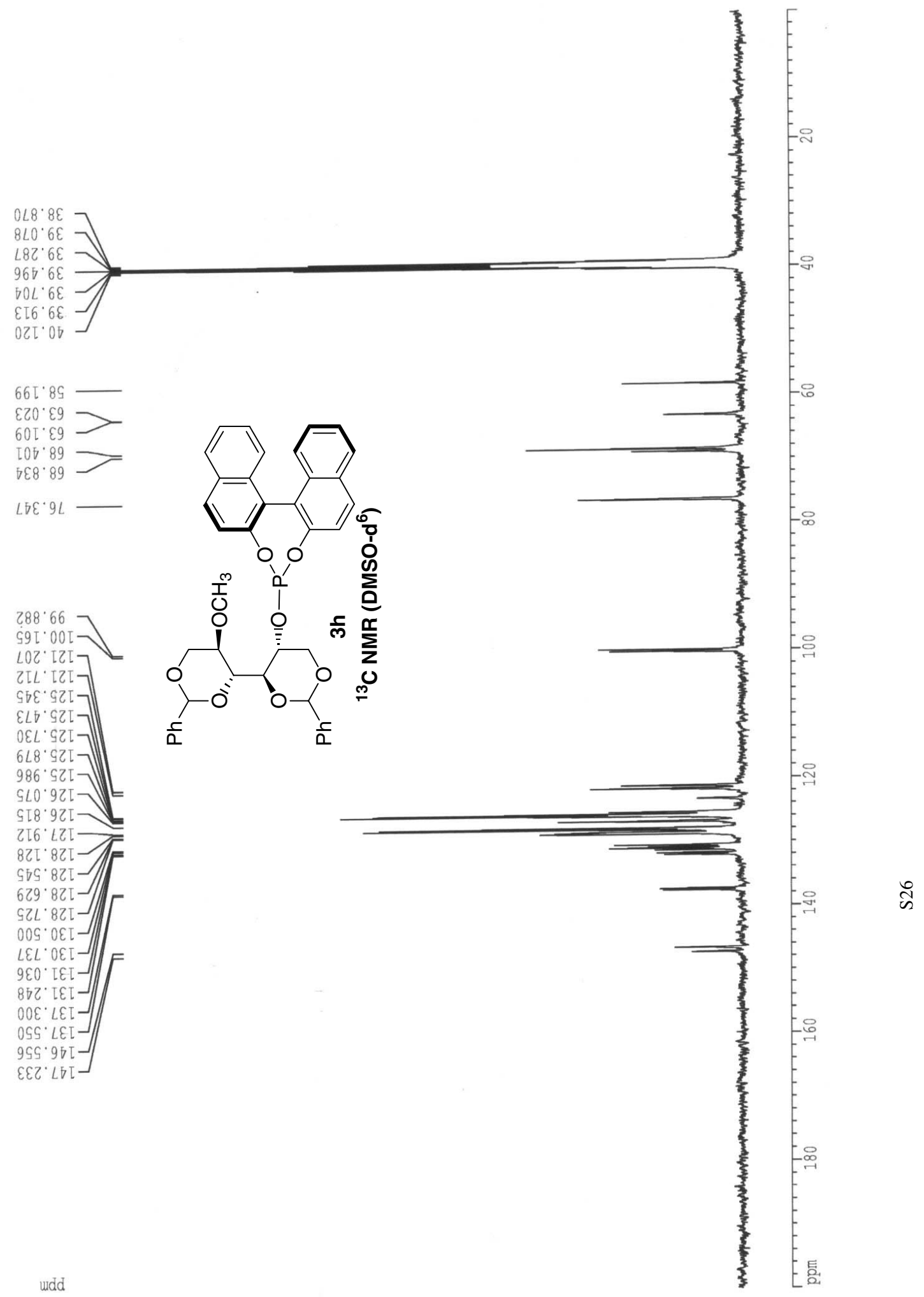




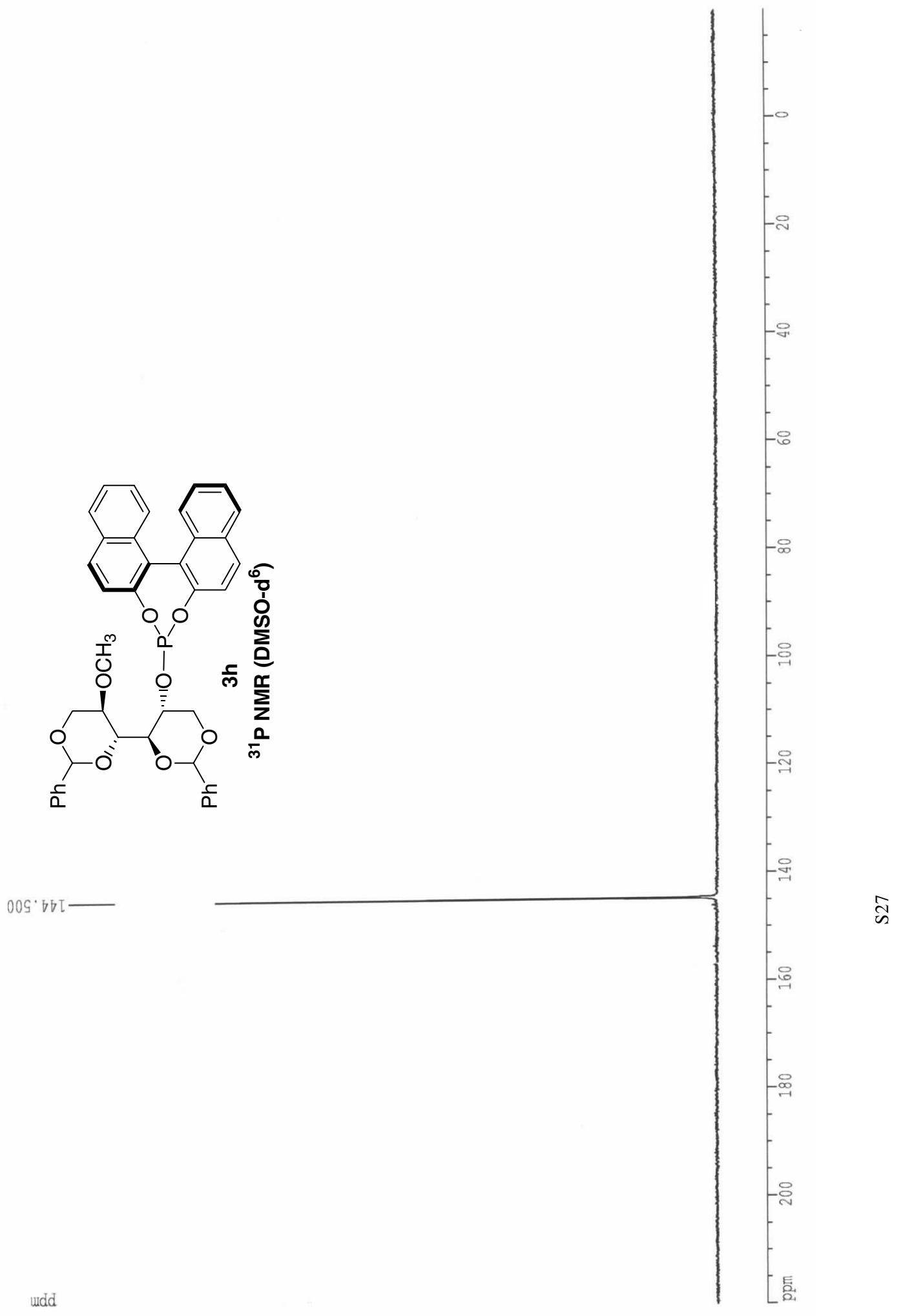



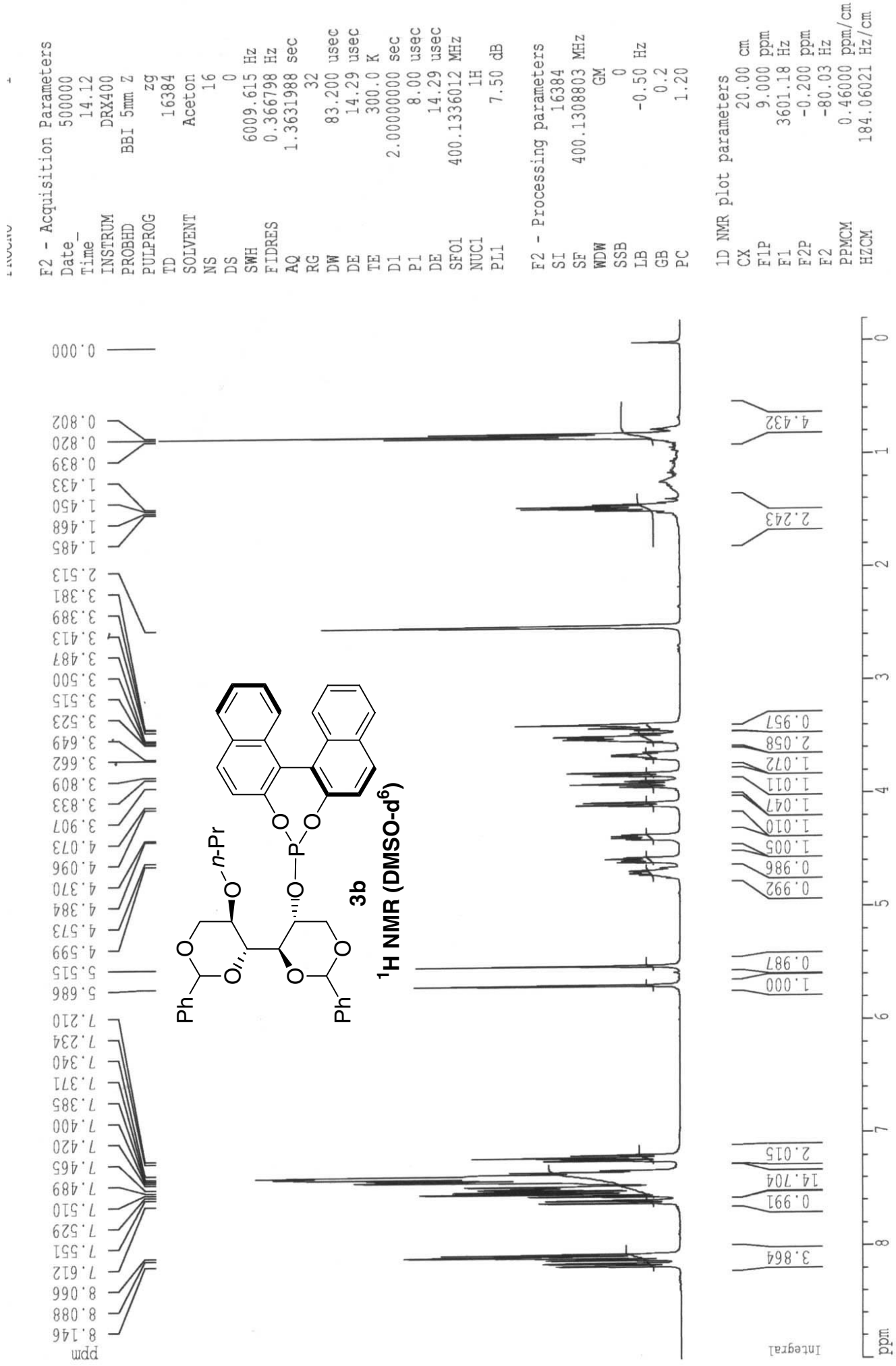

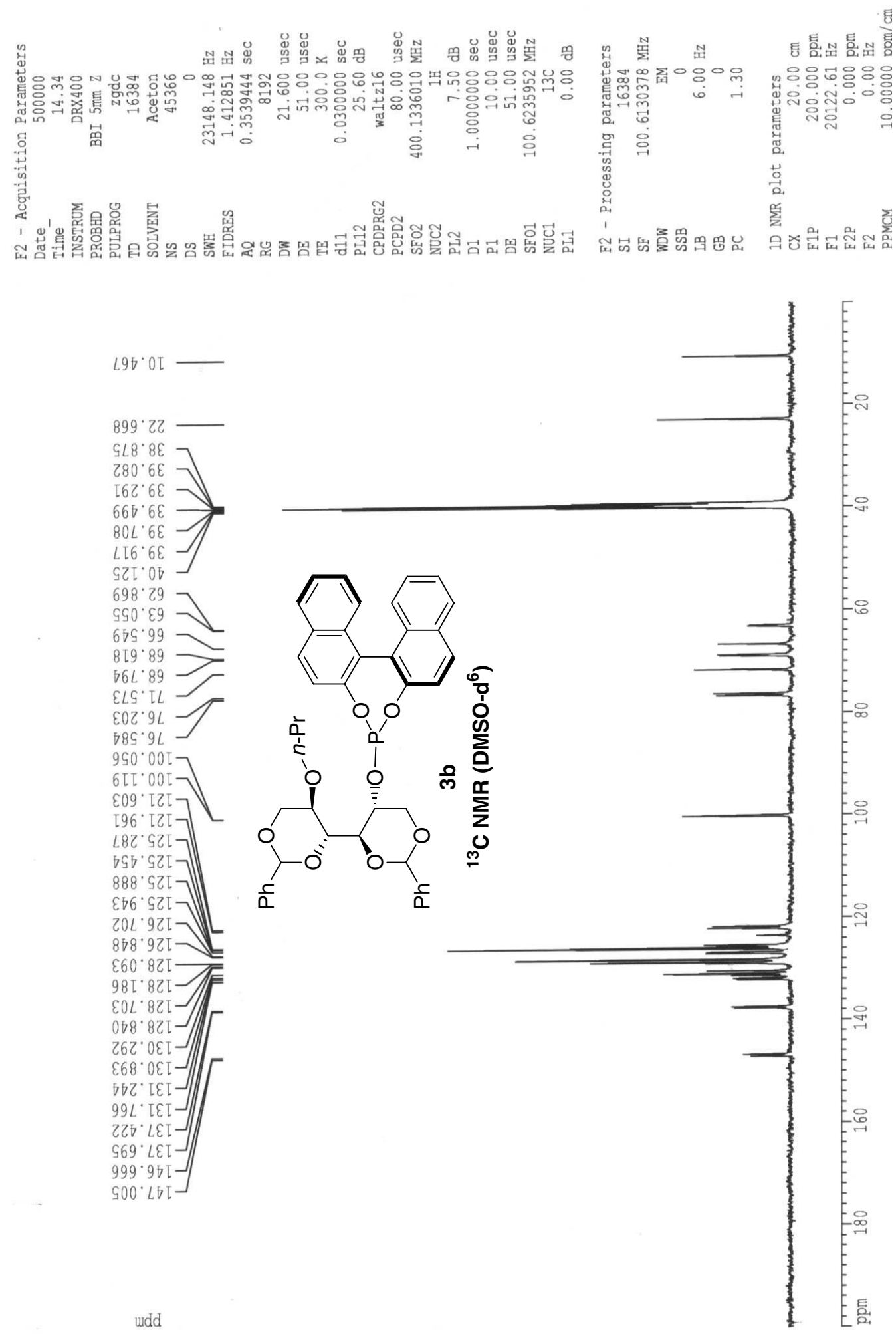

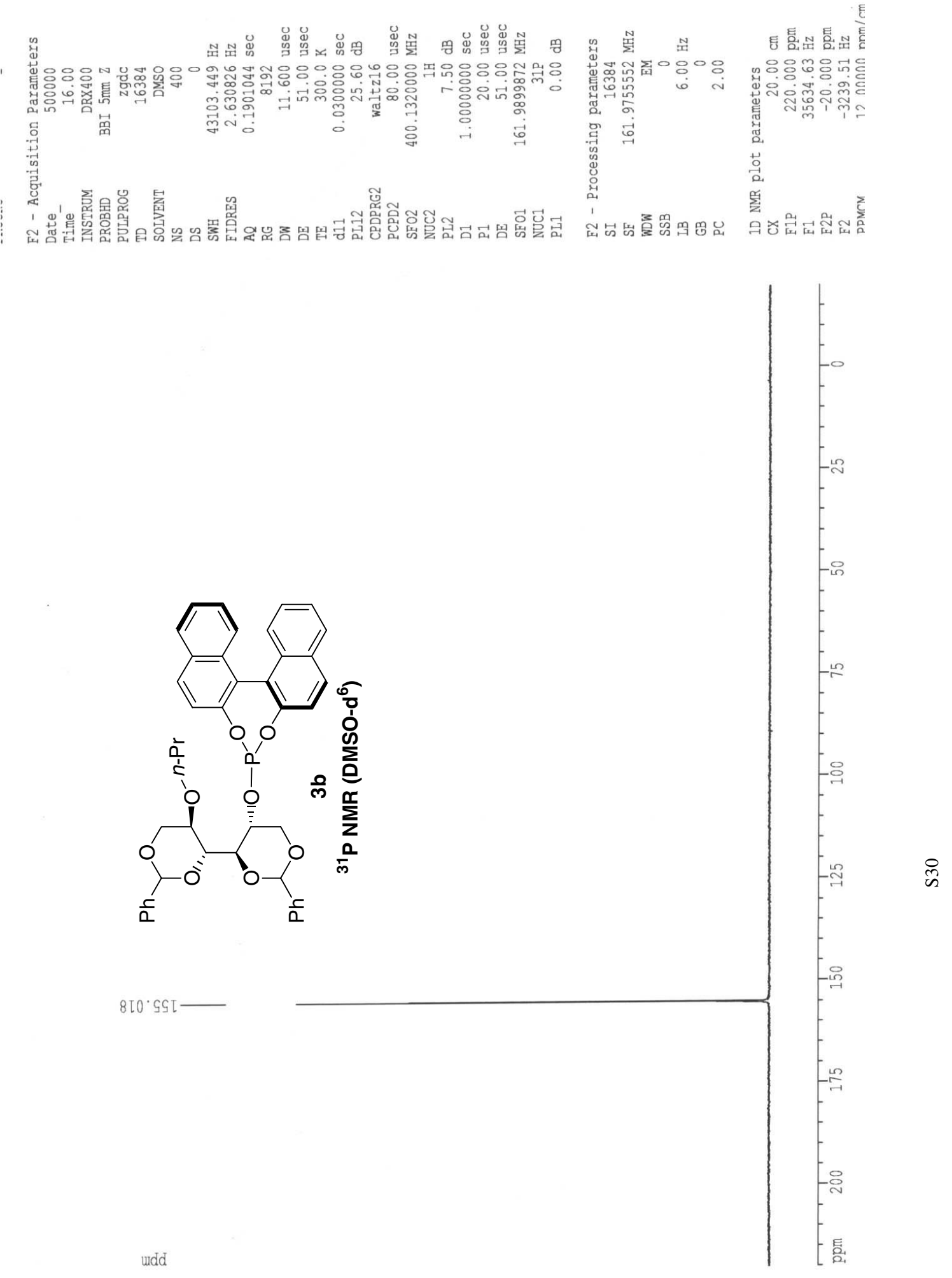


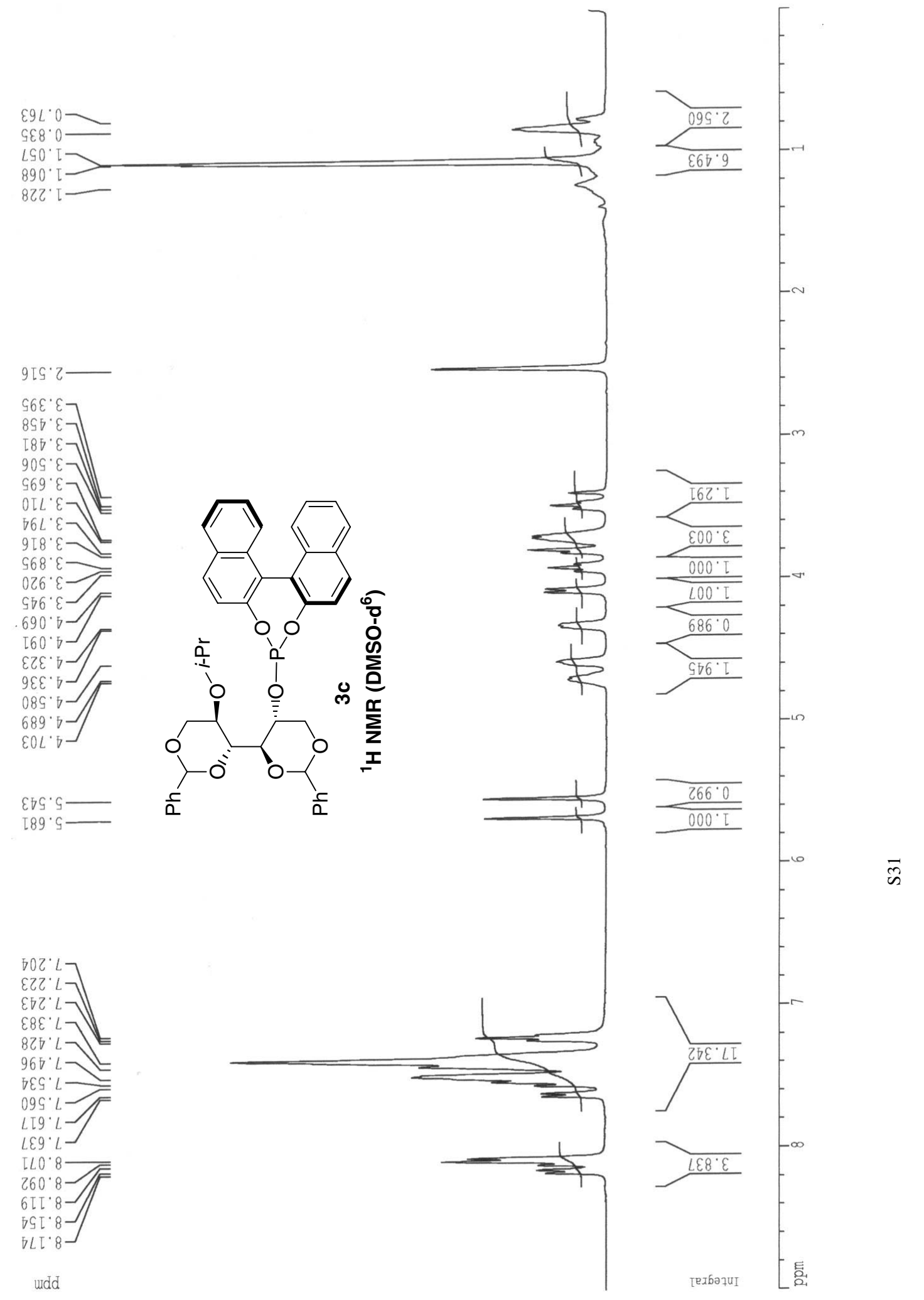



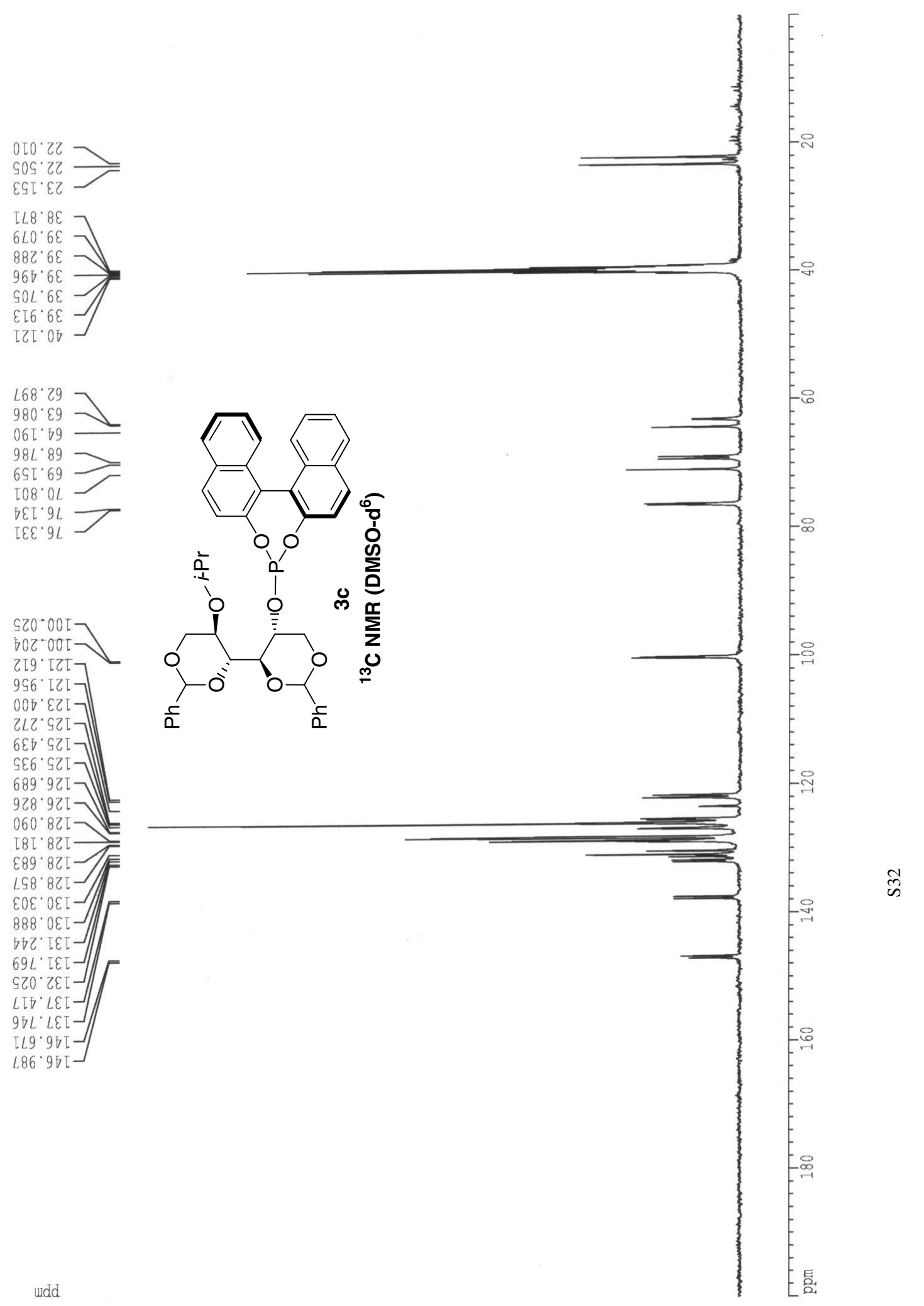


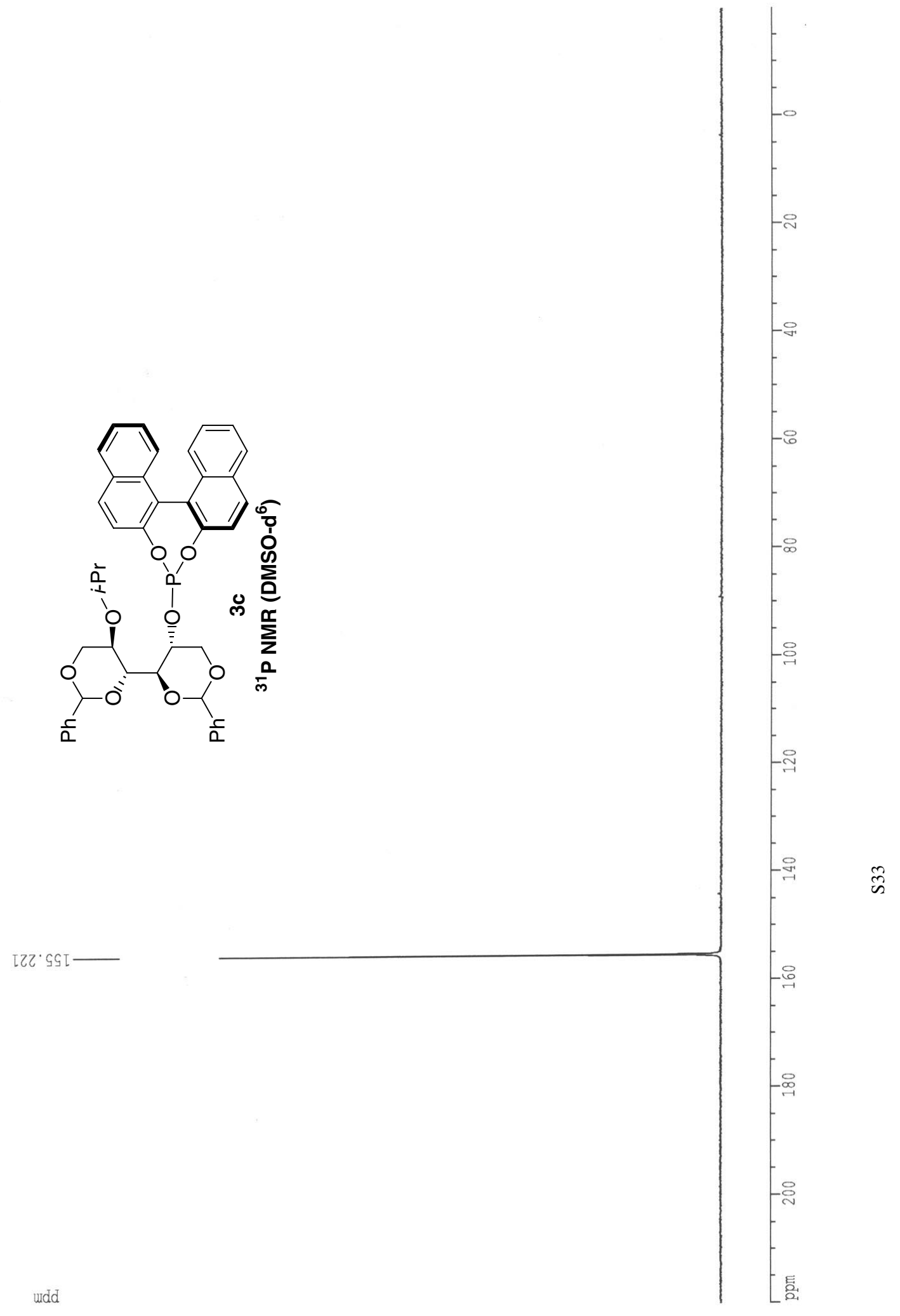




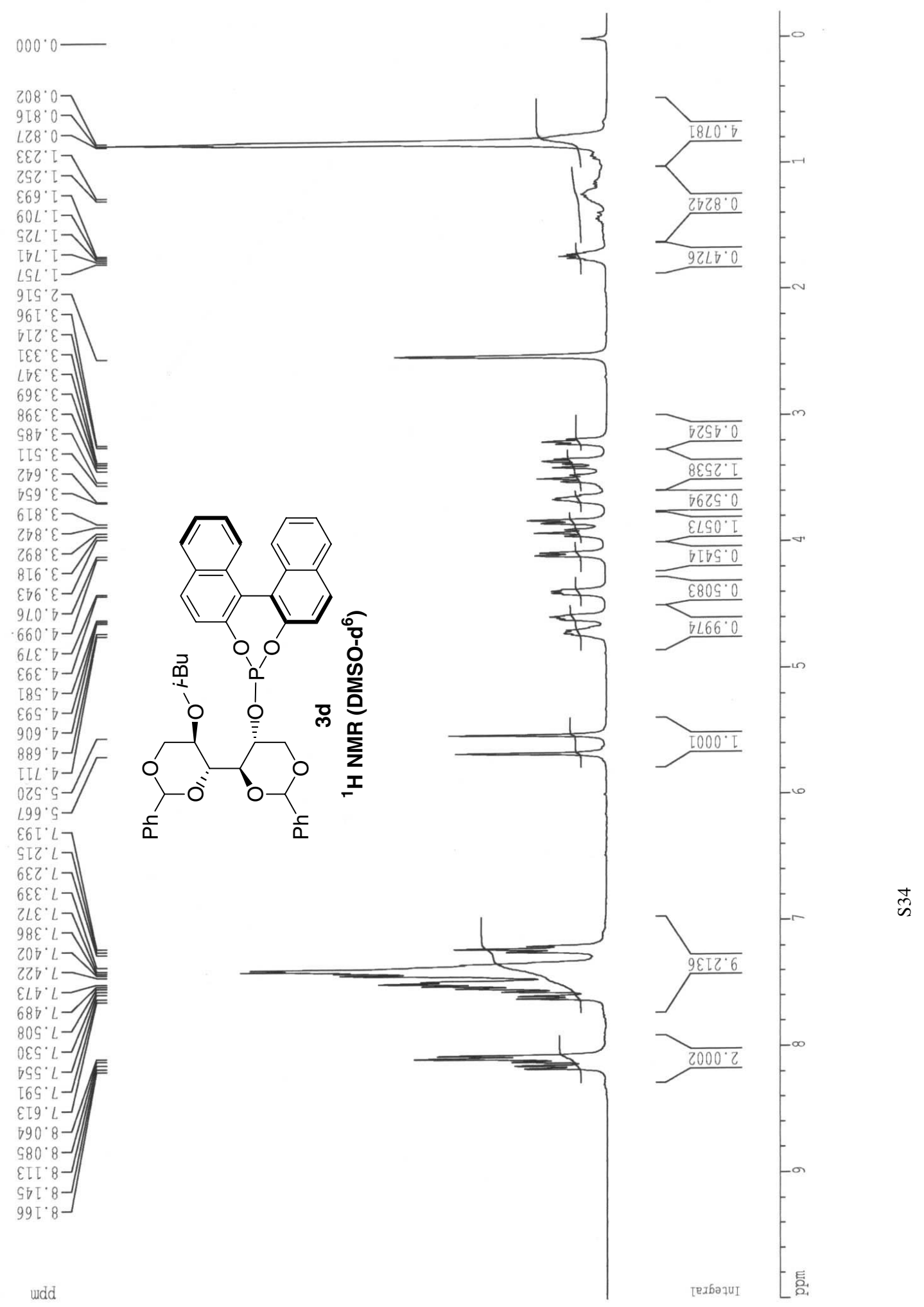



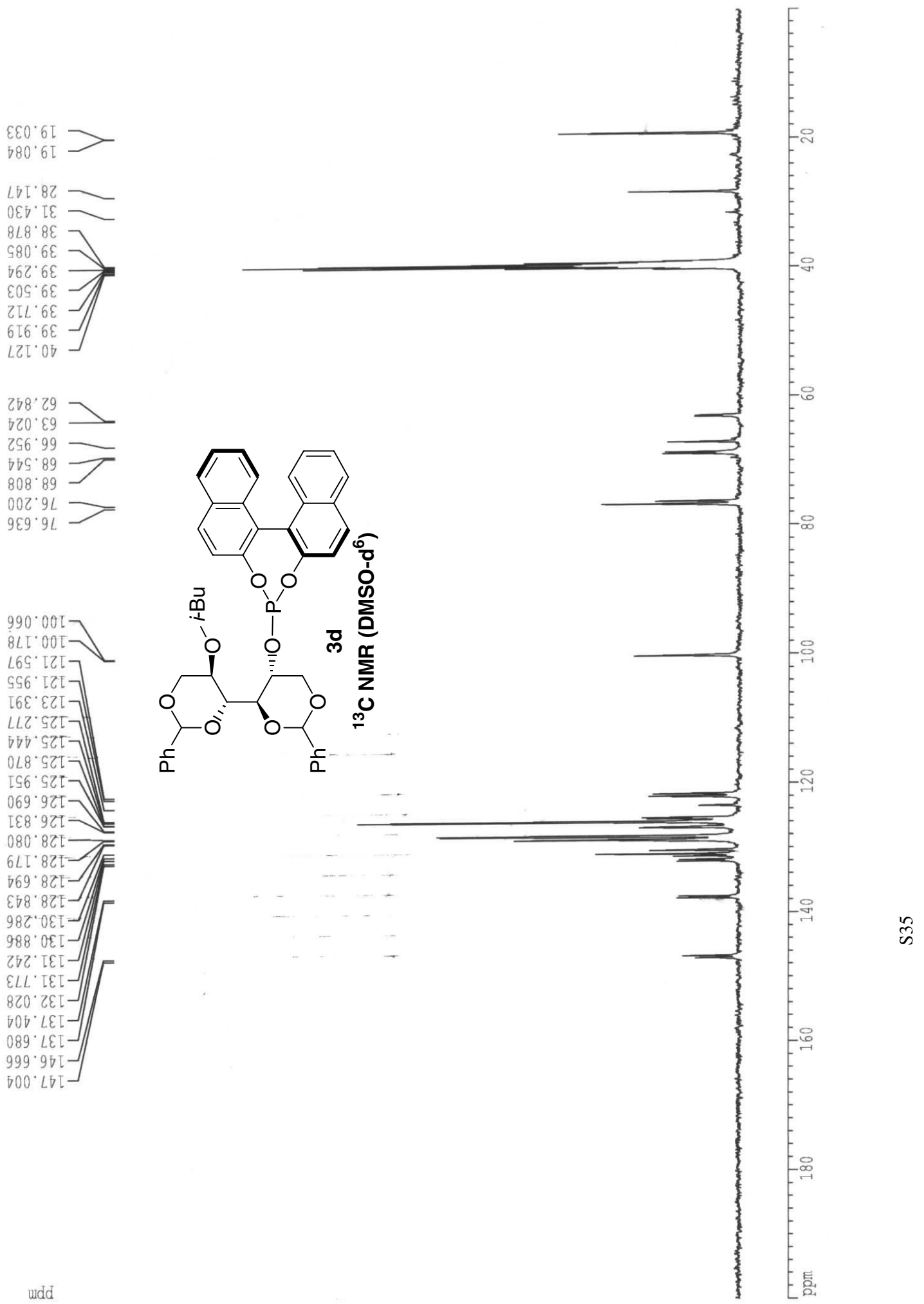


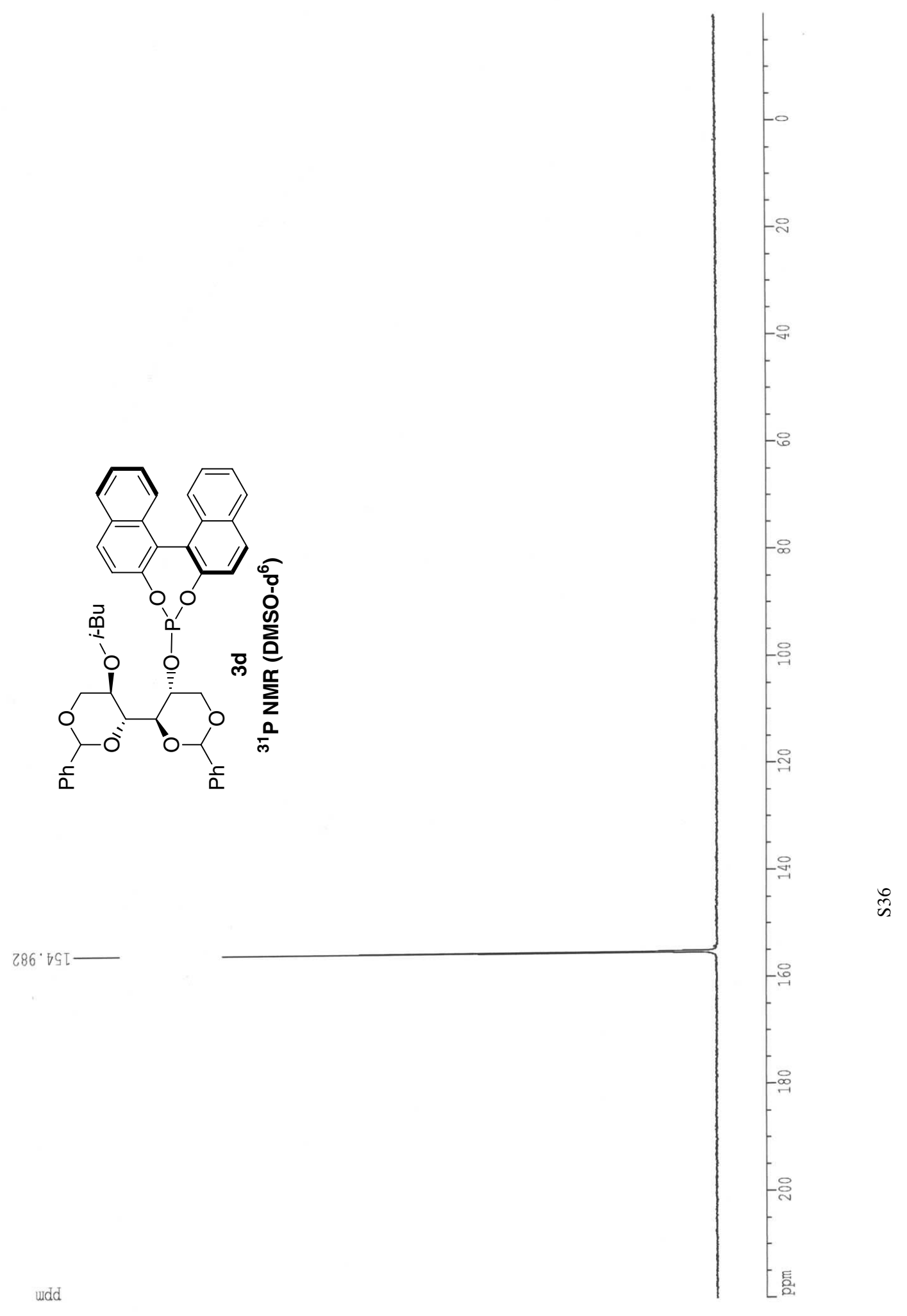


$000: 0-$

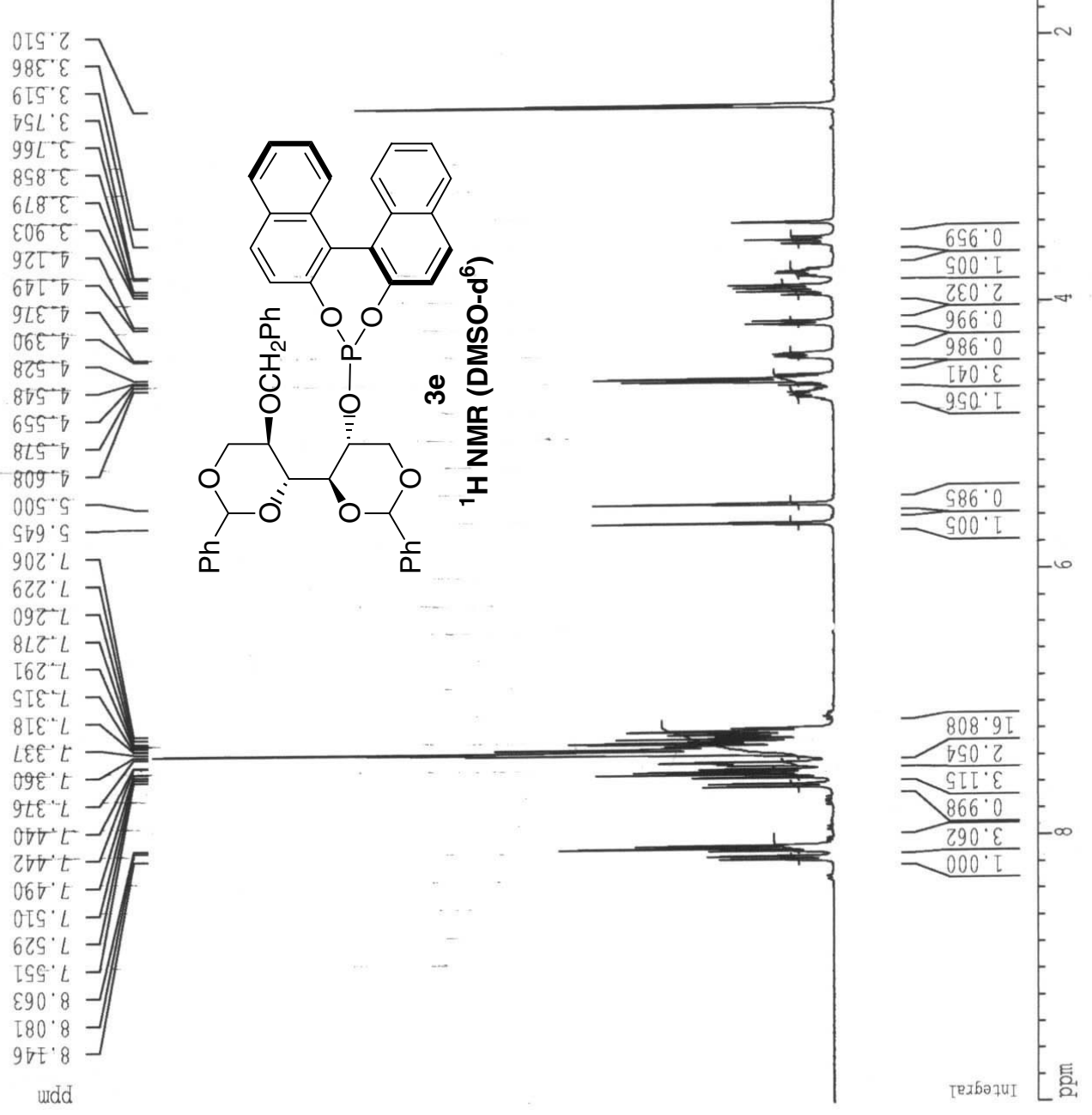




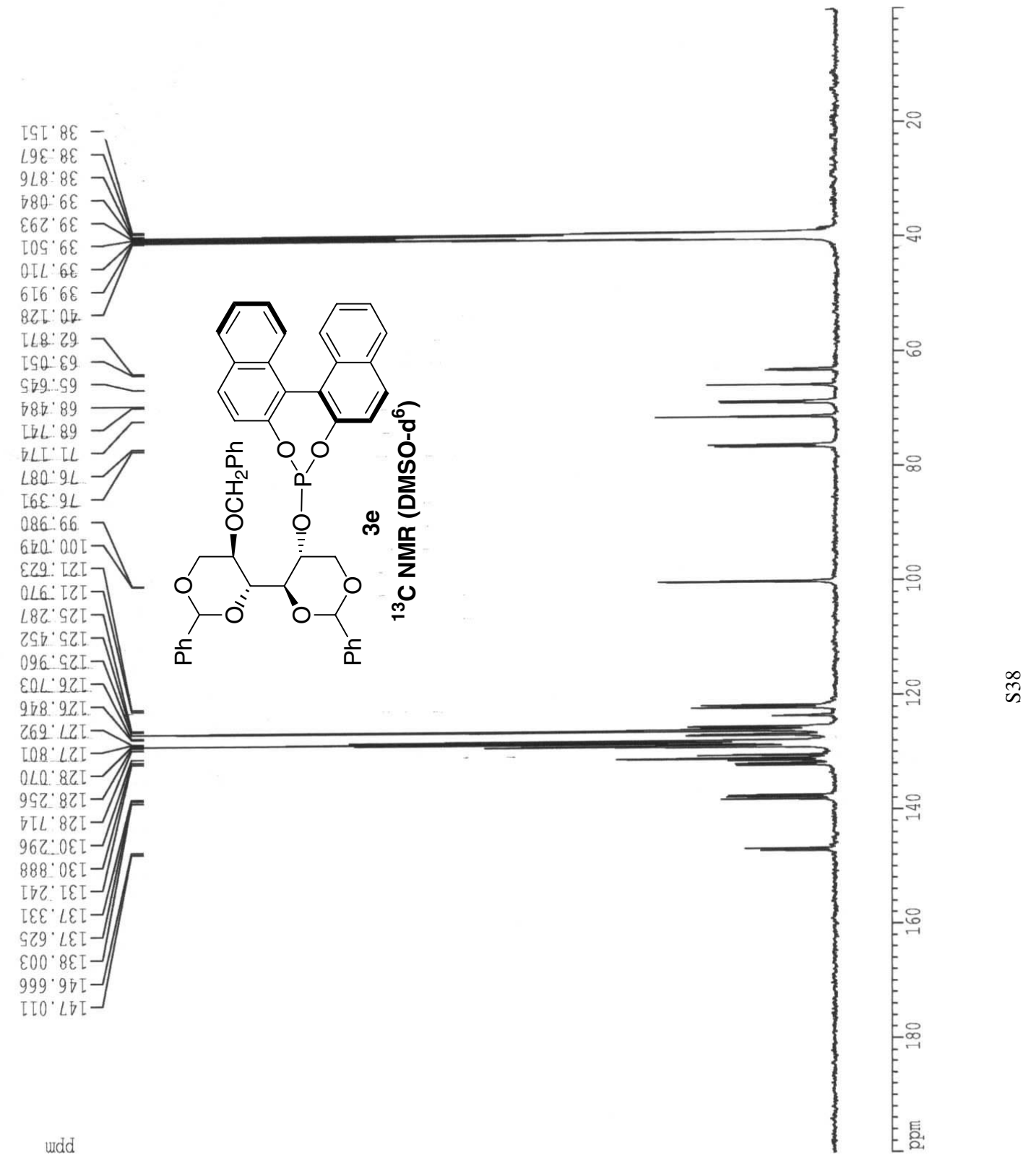




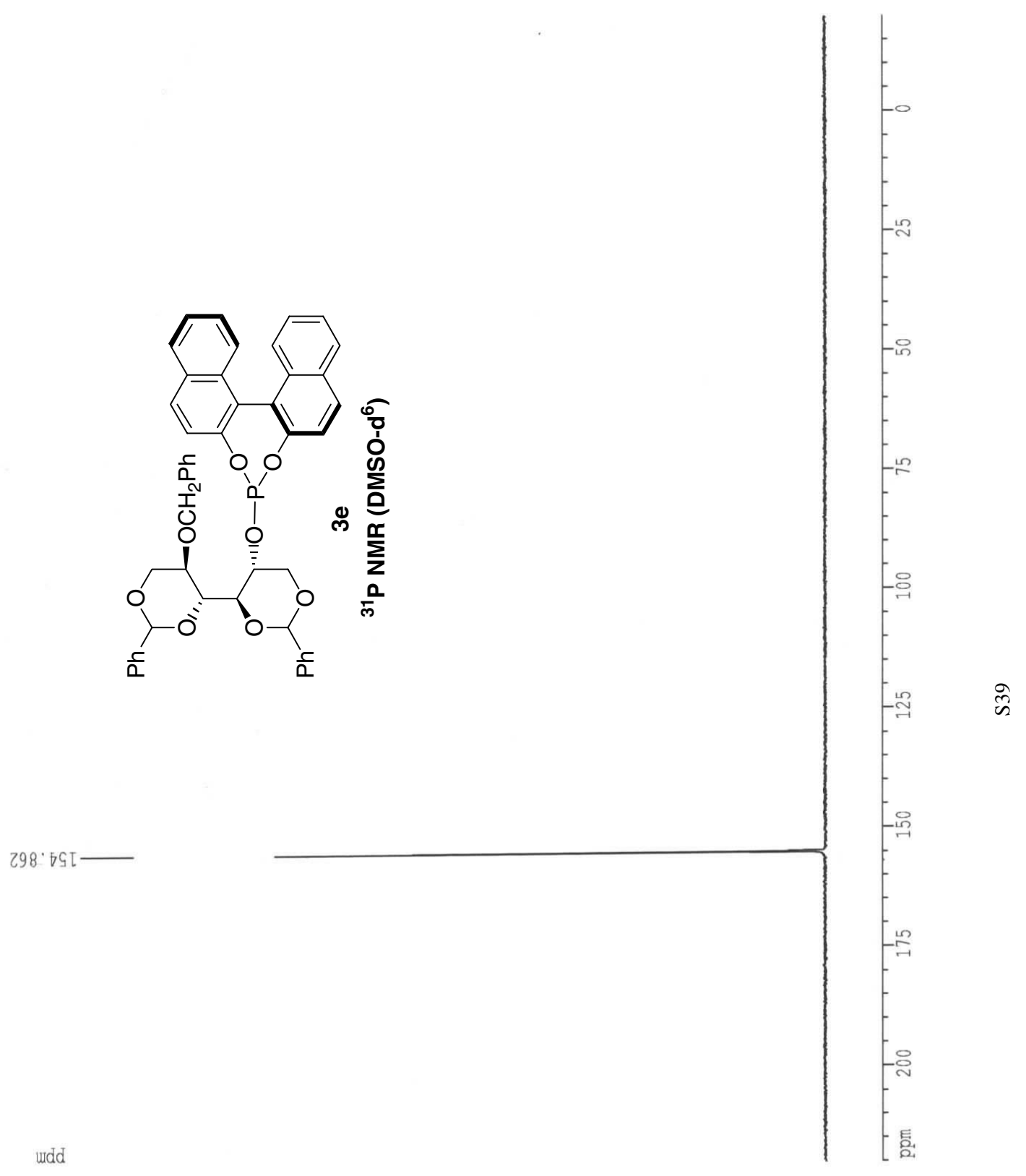



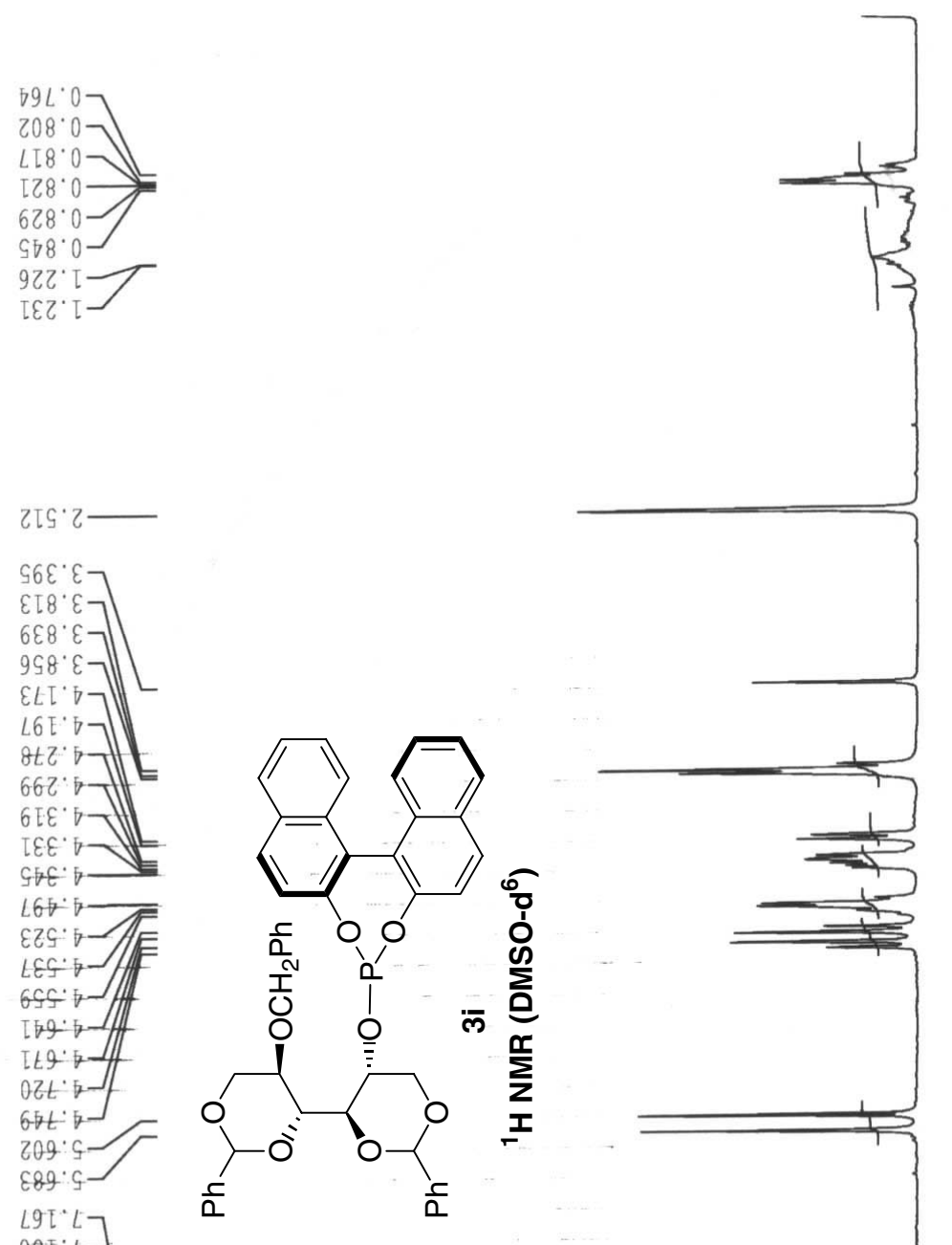

$\angle 9: L]$

$9 \varepsilon \tau^{\circ} L$

$\left.992^{\circ} L\right]$

$582^{\circ} L$

TOE $L]$

टE $\mathcal{E}^{\circ} L>$

ऽ $9 \varepsilon^{\circ} L$

OTf't

$8 Z T^{\circ} L$

$980^{\circ} L$

$50 \mathrm{~S}^{\circ} \mathrm{L}-$

$\angle z 9^{\circ}:-1 / F$

$0 Z L^{\circ} L-$

$2 \nabla L \cdot L$

$290^{\circ} 8-$

$280^{\circ} 8$

$\varepsilon 60^{\circ} 8-$

$09[: 8]$

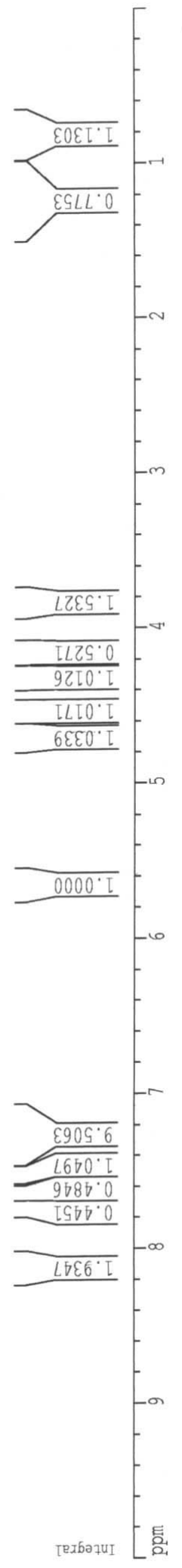




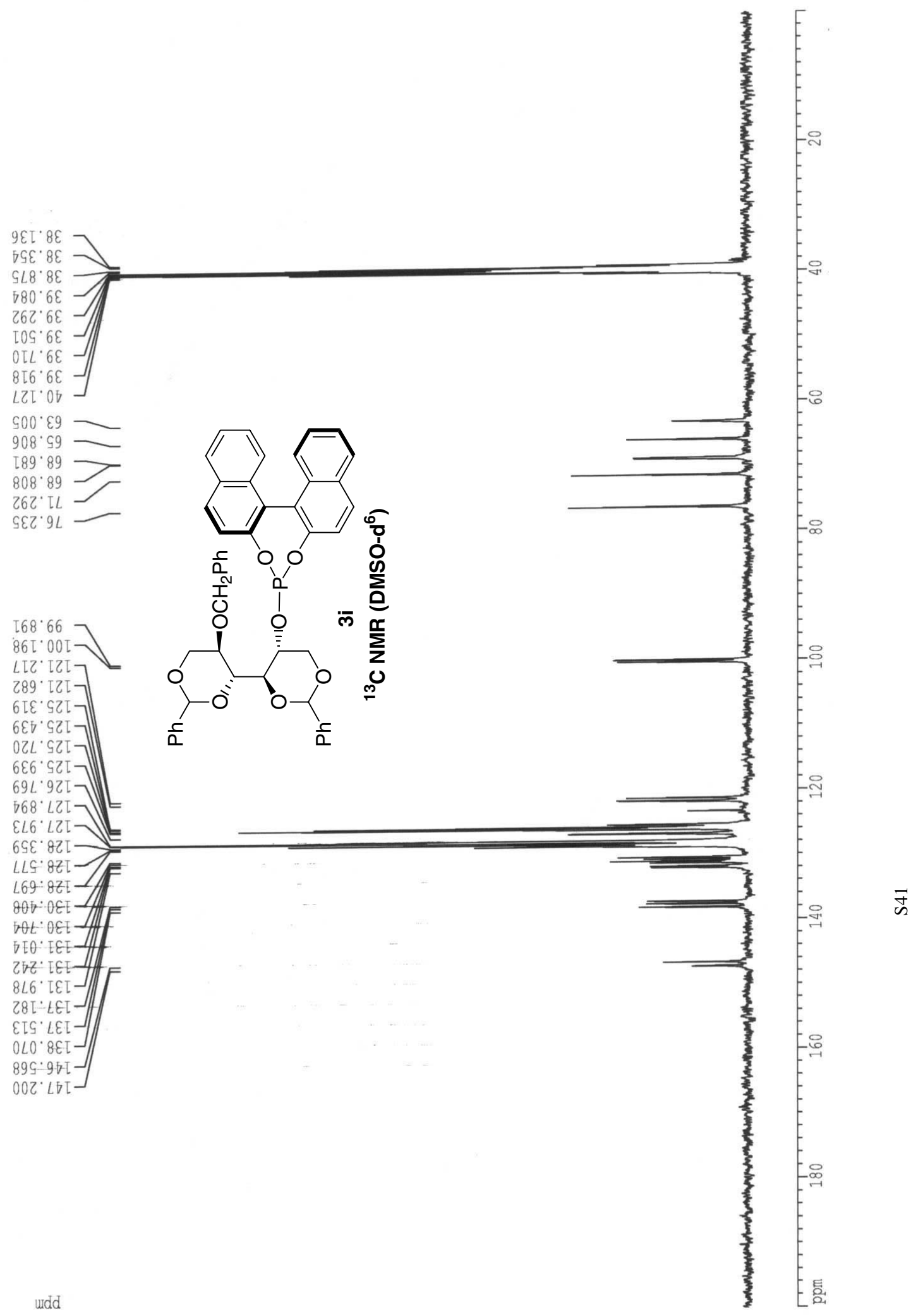




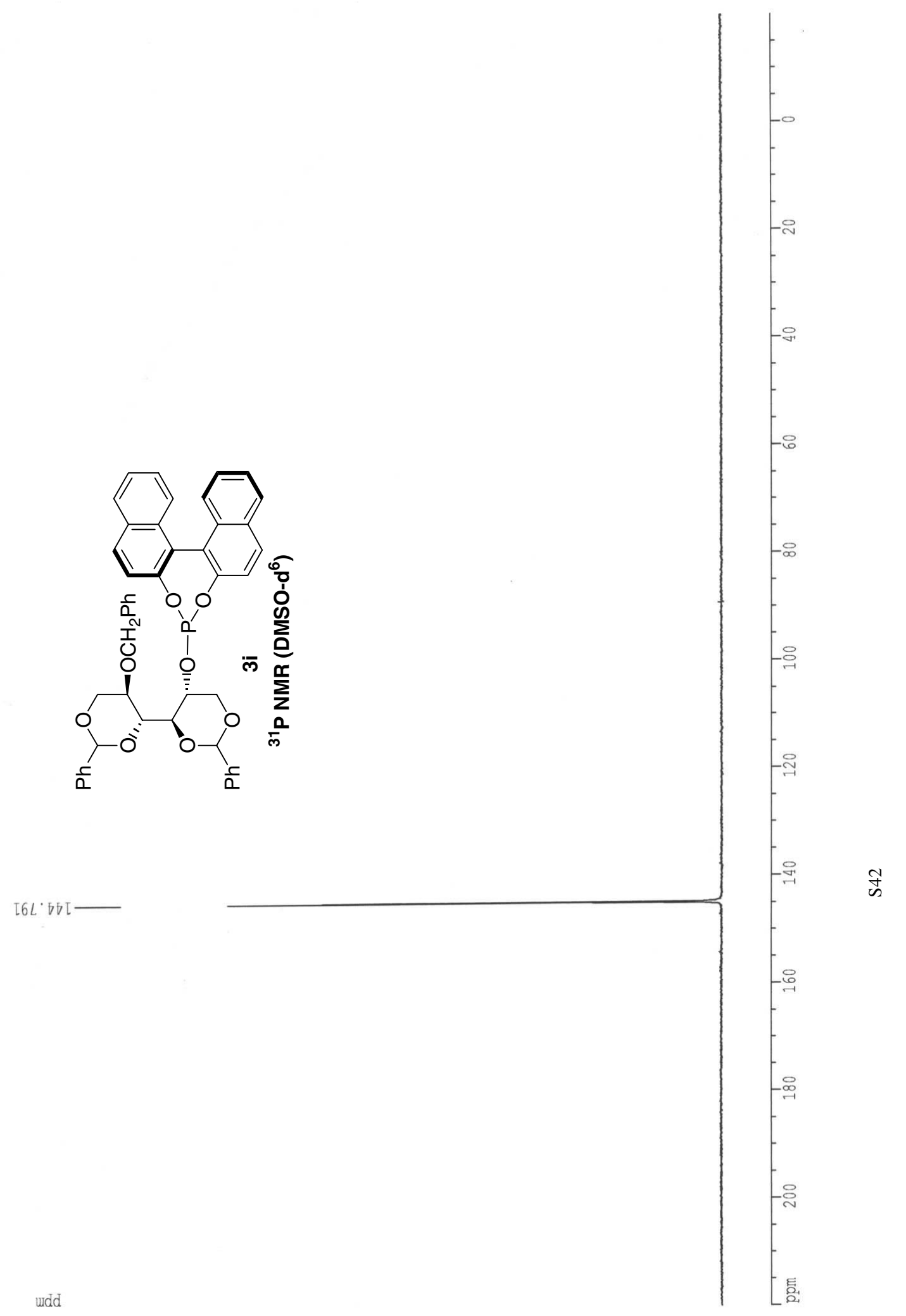




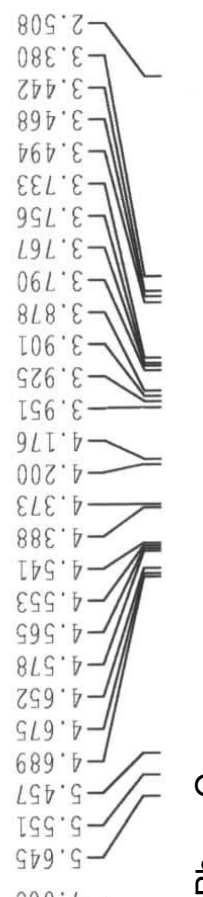

$890^{\circ} \mathrm{L}$
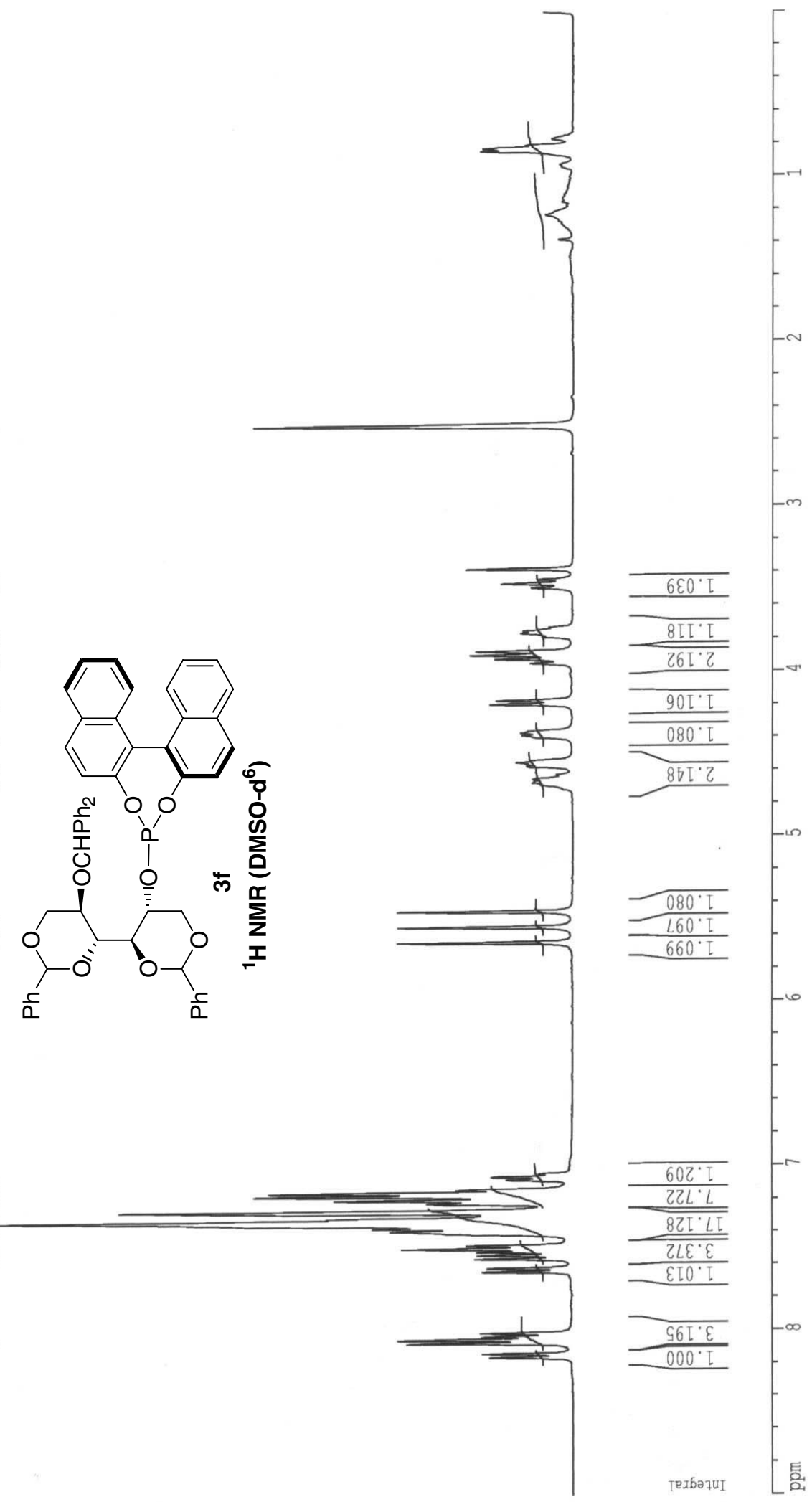

$98[\circ$

$602^{\circ} L$

IEZ' $L$

$\angle L Z Z^{\circ} L$

$782^{\circ} L$

Z२ह $L$

$8 \varepsilon \varepsilon^{\circ} L-$

$28 \varepsilon^{\circ} L$

$66 \varepsilon^{\circ} L-$

280 L

$90 \mathrm{~S}^{\circ} \mathrm{L}-$

StG. $L$

L9S ${ }^{\circ} \mathrm{L}$

$690.8-$

udd 


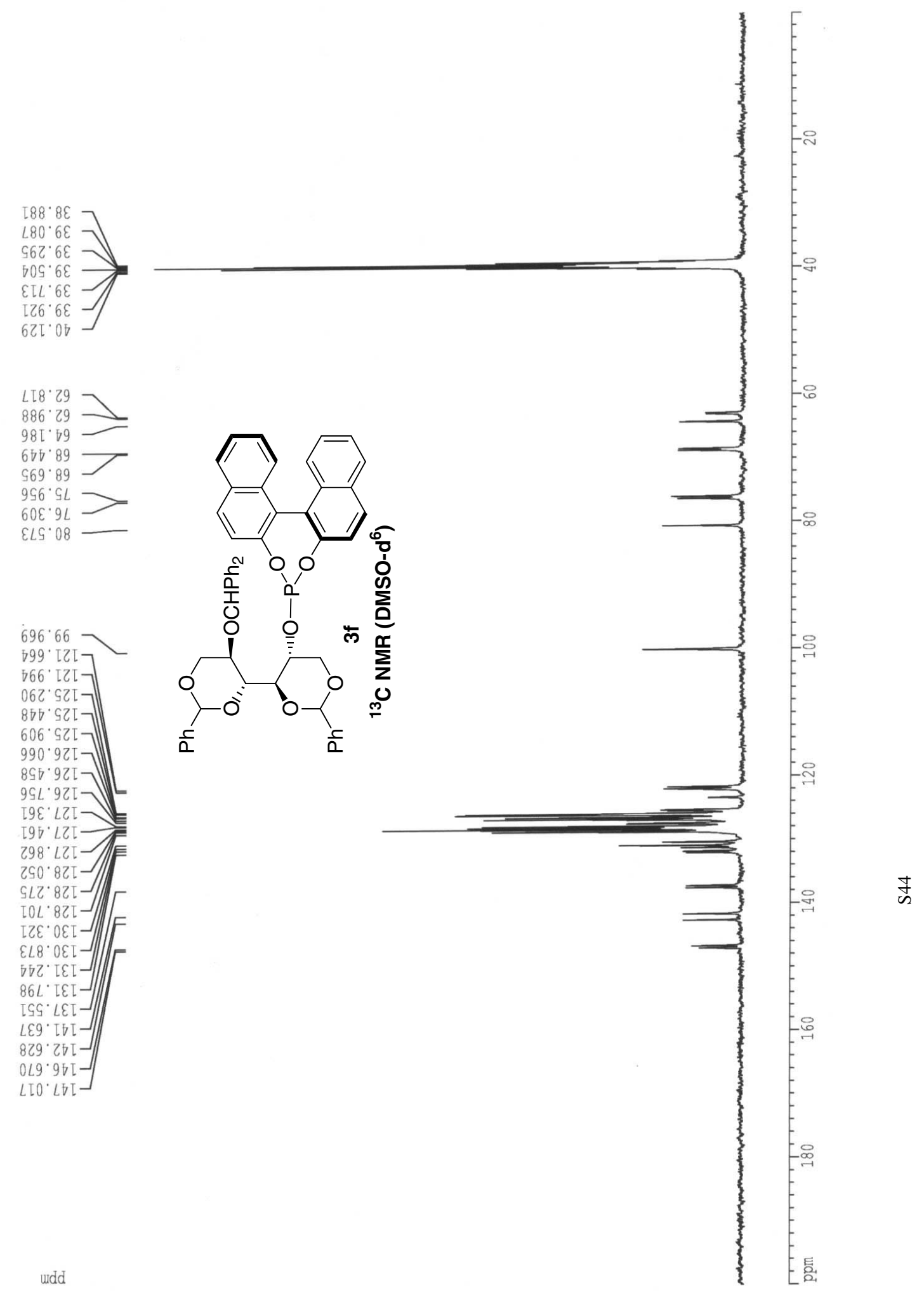




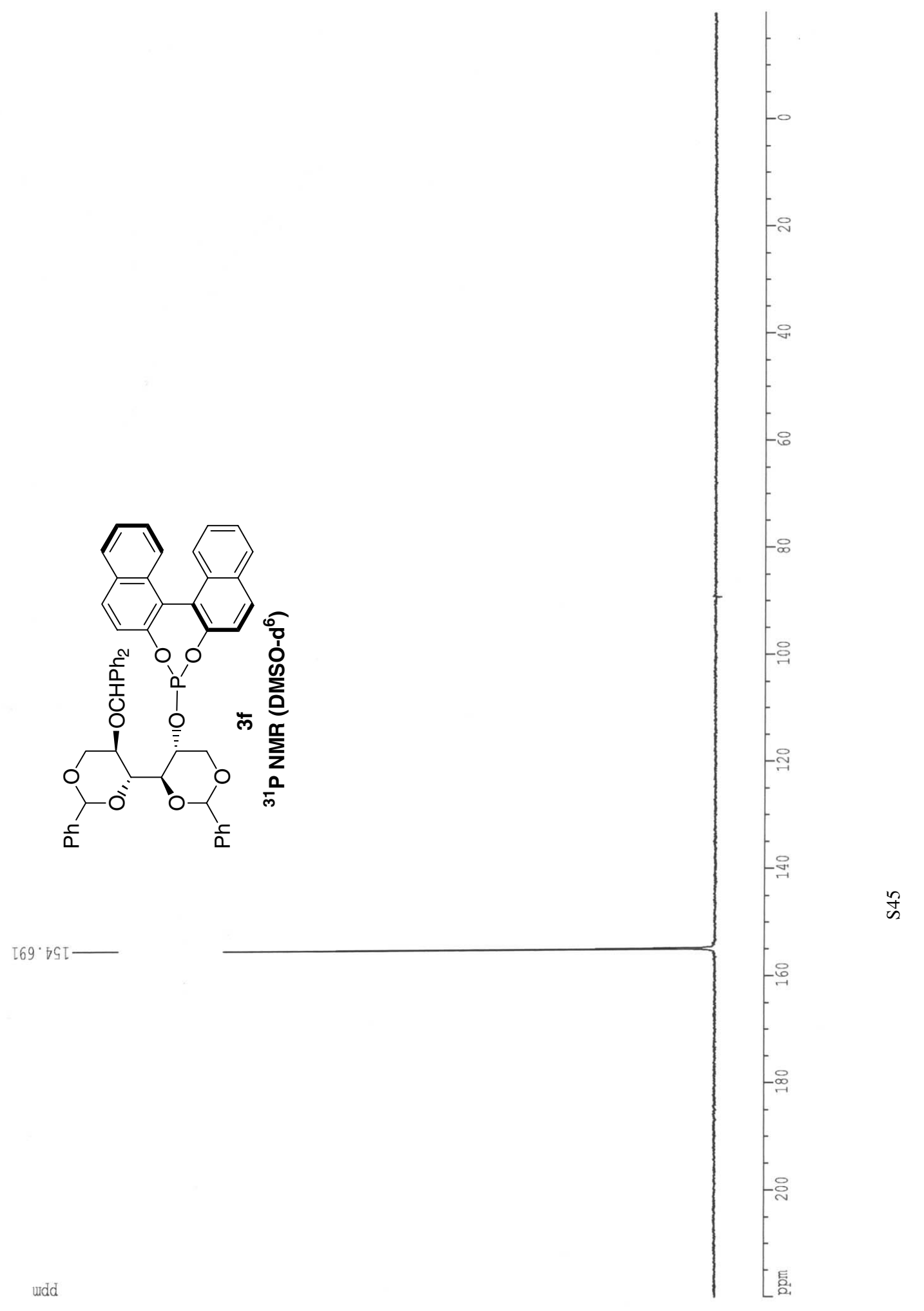



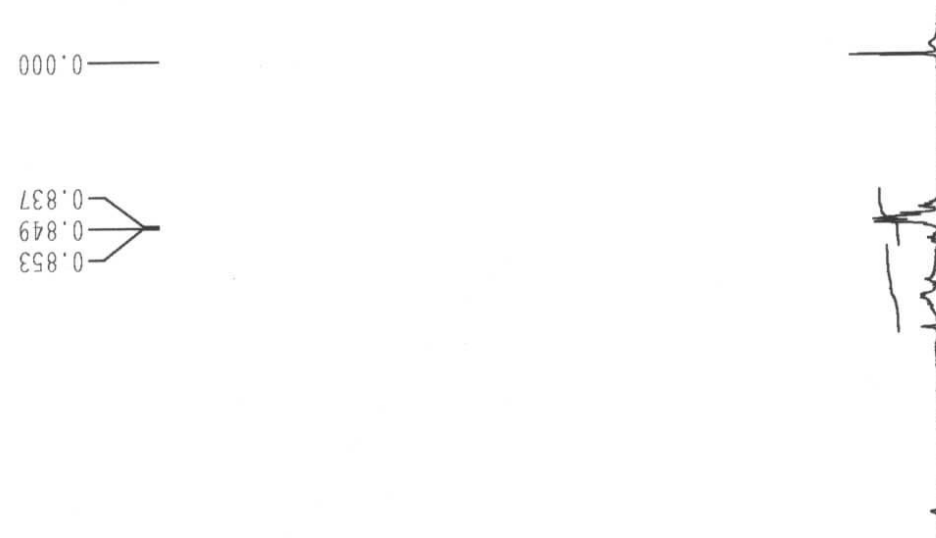

LOS

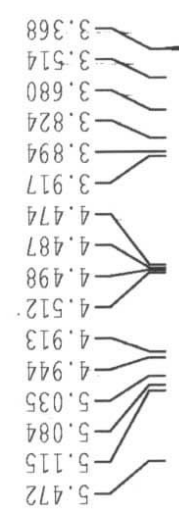

$\varepsilon 90^{\circ} \mathrm{L}$

$\left.6 L 0^{\circ} L\right]$

$\varepsilon 6[\cdot L]$
$6\left[\tau^{\circ} L\right]$

$\left.90 \varepsilon^{\circ} L\right]$

$92 \varepsilon^{\circ} L$

$S \pitchfork \varepsilon^{\circ} L 7$

โรE. $L$ -

TOF $L$

SOF $L-$

G

$\left.880^{\circ} \cdot L-1\right]$

OZS. L. F

EES $L-$

$009^{\circ} \mathrm{L}_{-}$

$229^{\circ} \mathrm{L}-$

$090^{\circ} 8-$

G5: $80.8-$

udd
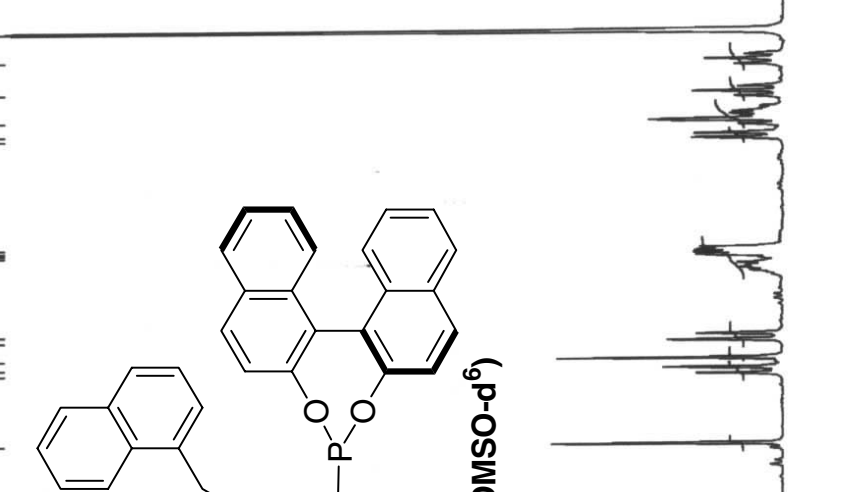

1
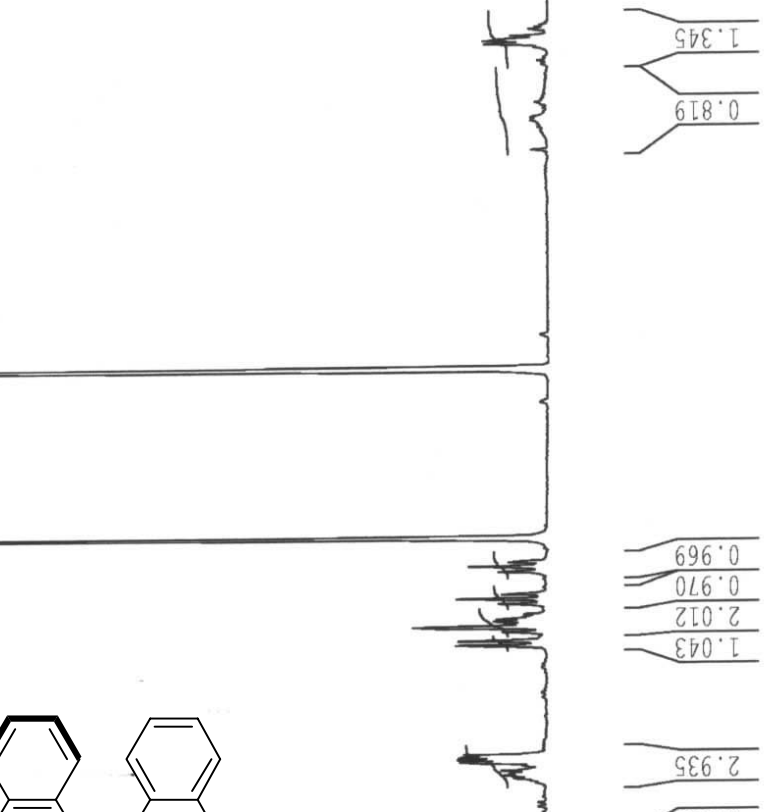

11

$\|_{\text {\% }}^{\circ}$

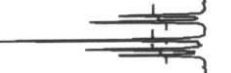

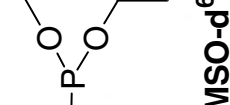

$\longrightarrow+\frac{596.0}{1+3}$

일 율
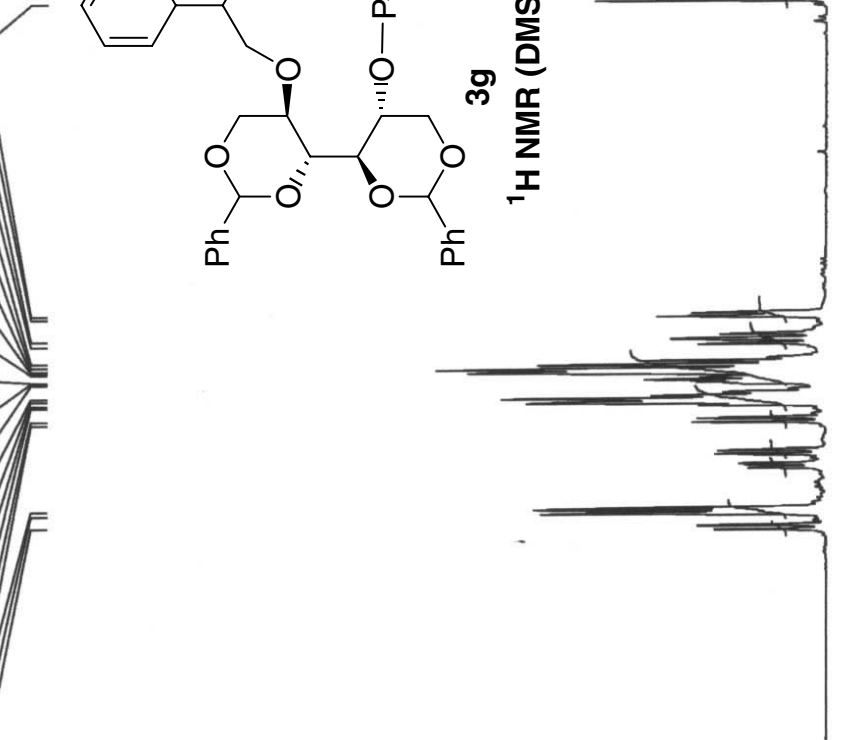<smiles>[3H]C1OCC[C@@H](C2CCOC([CH])O2)O1</smiles>

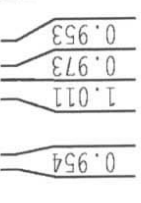
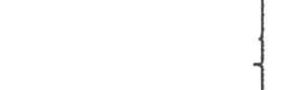

.

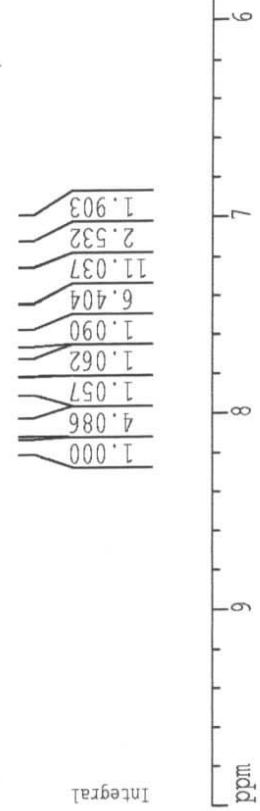

诂 


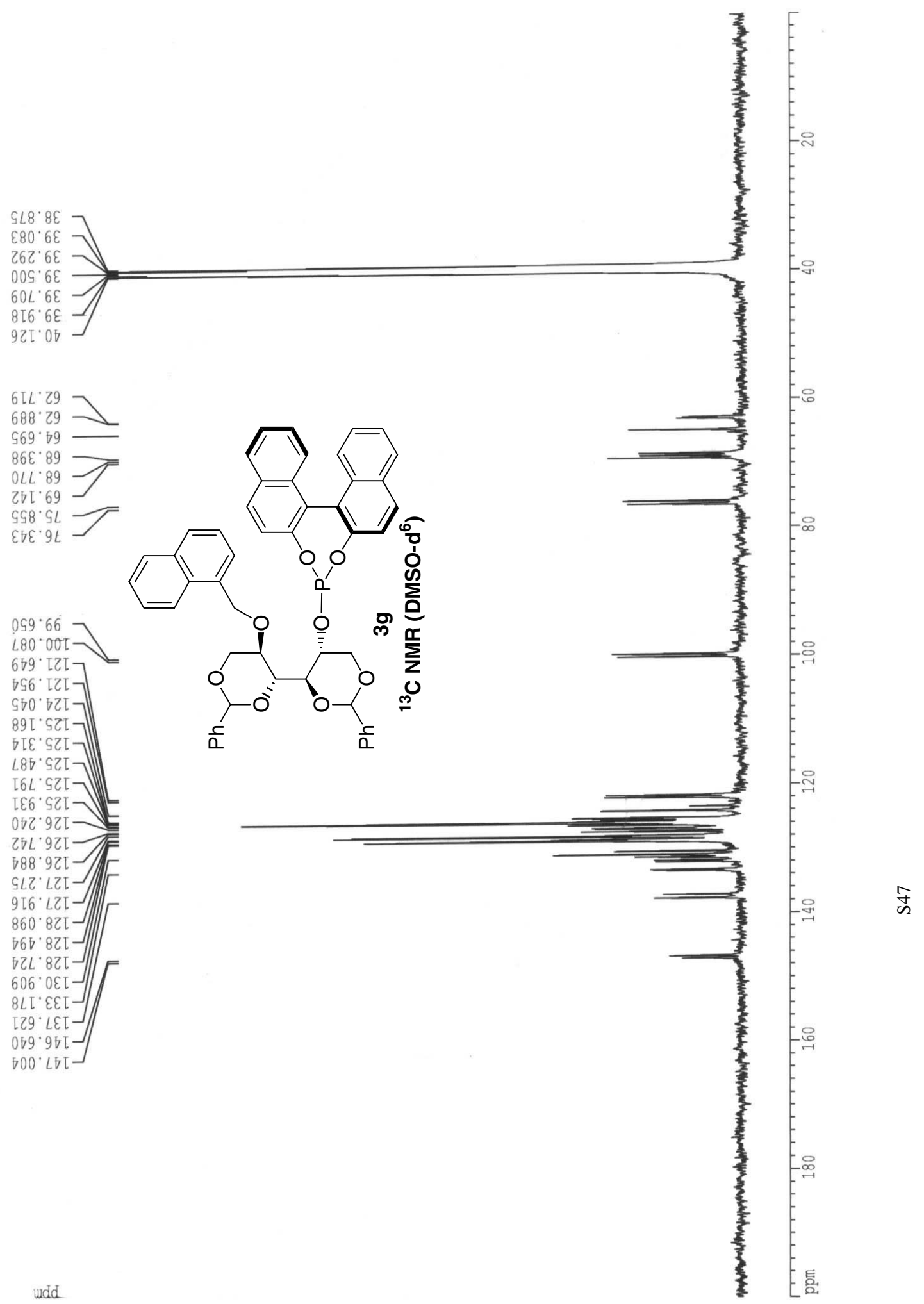




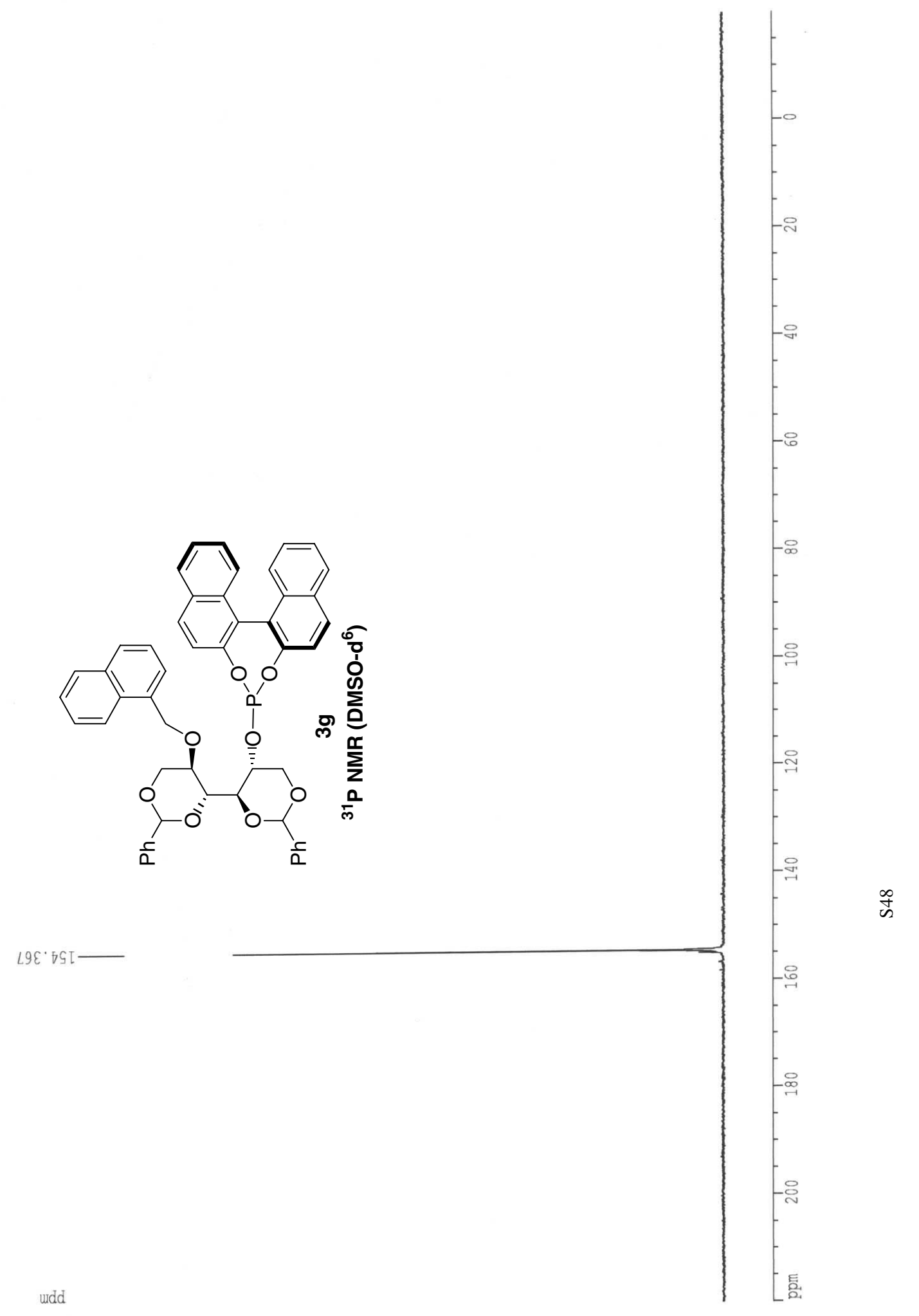




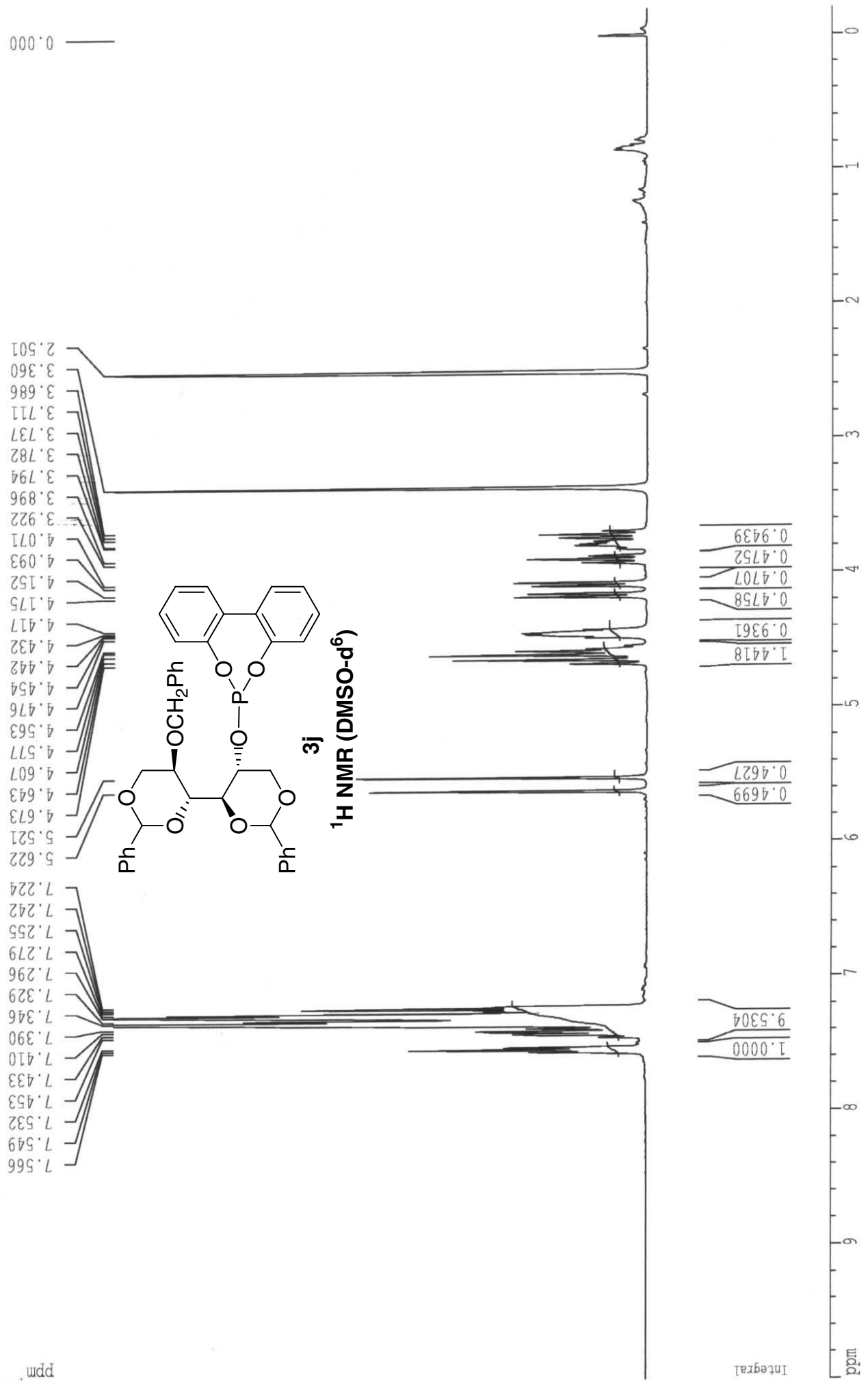

g 


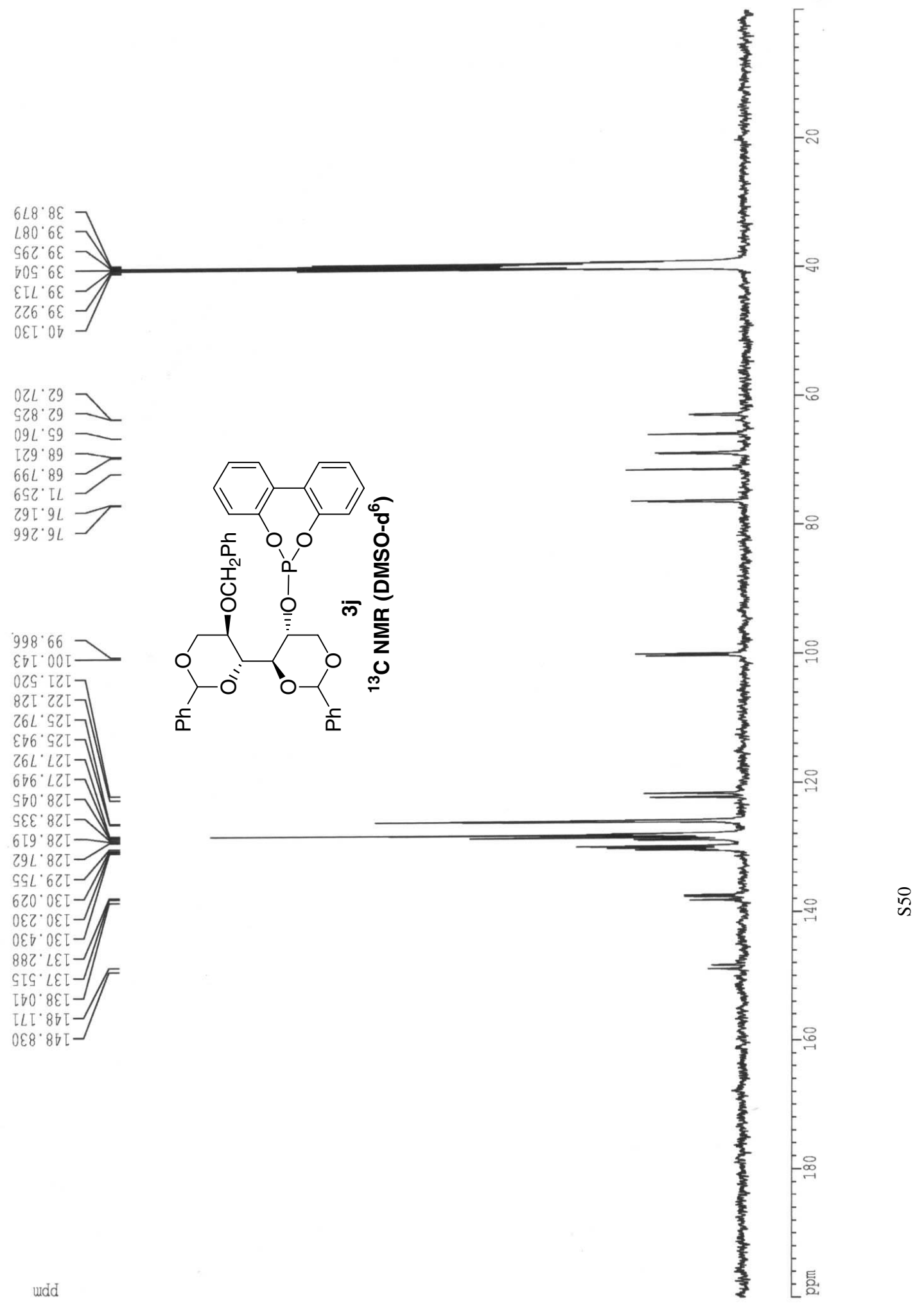




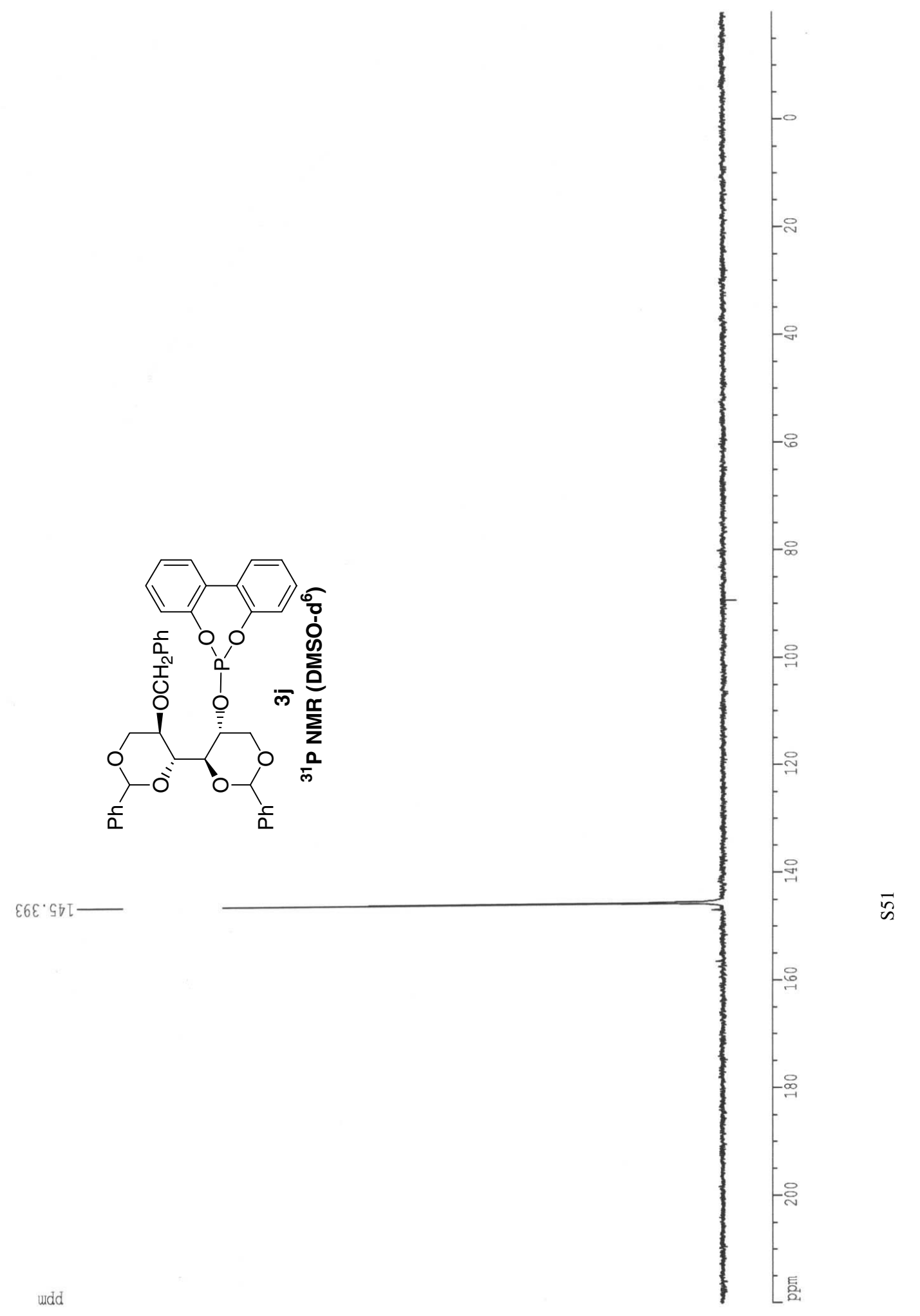




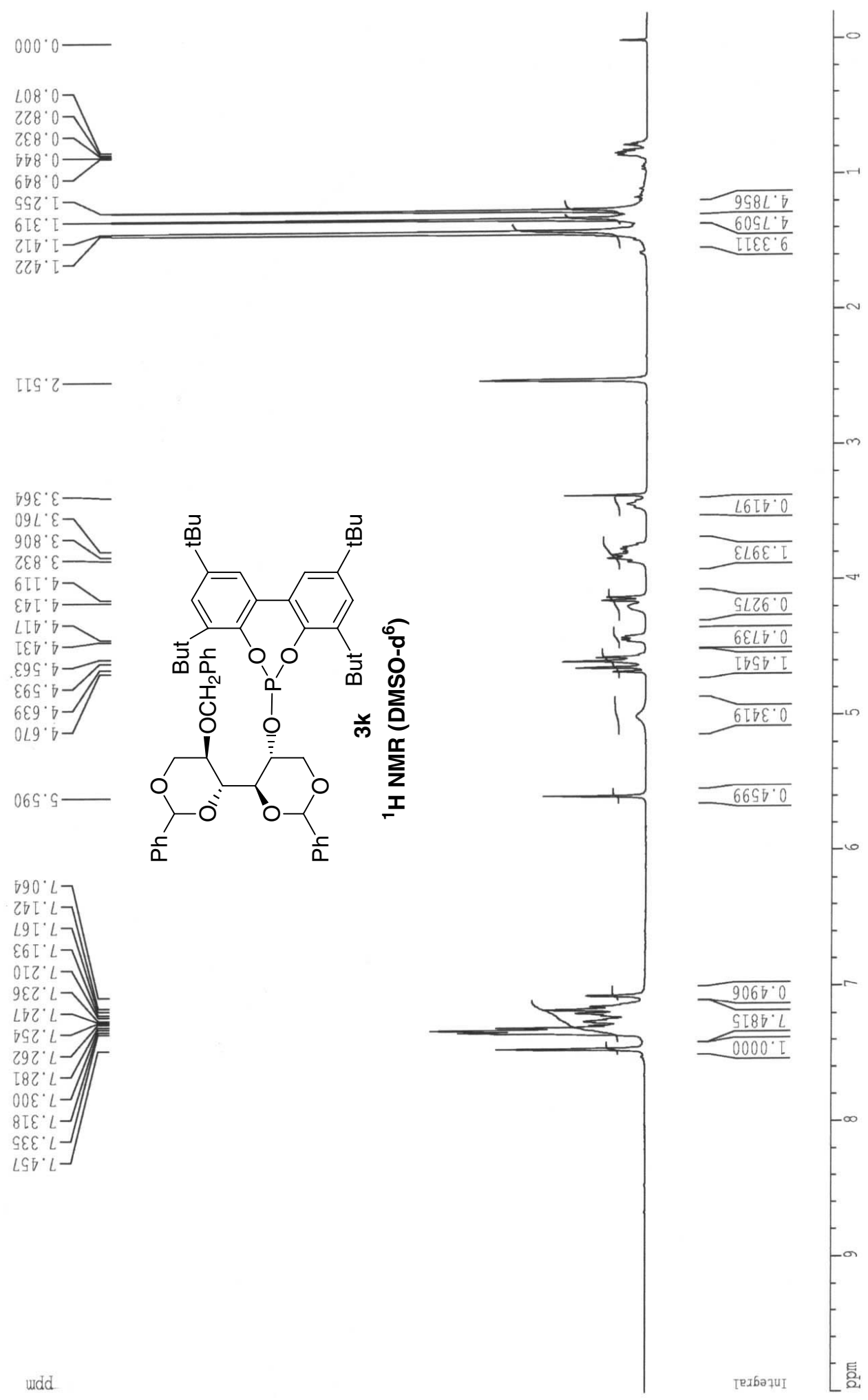




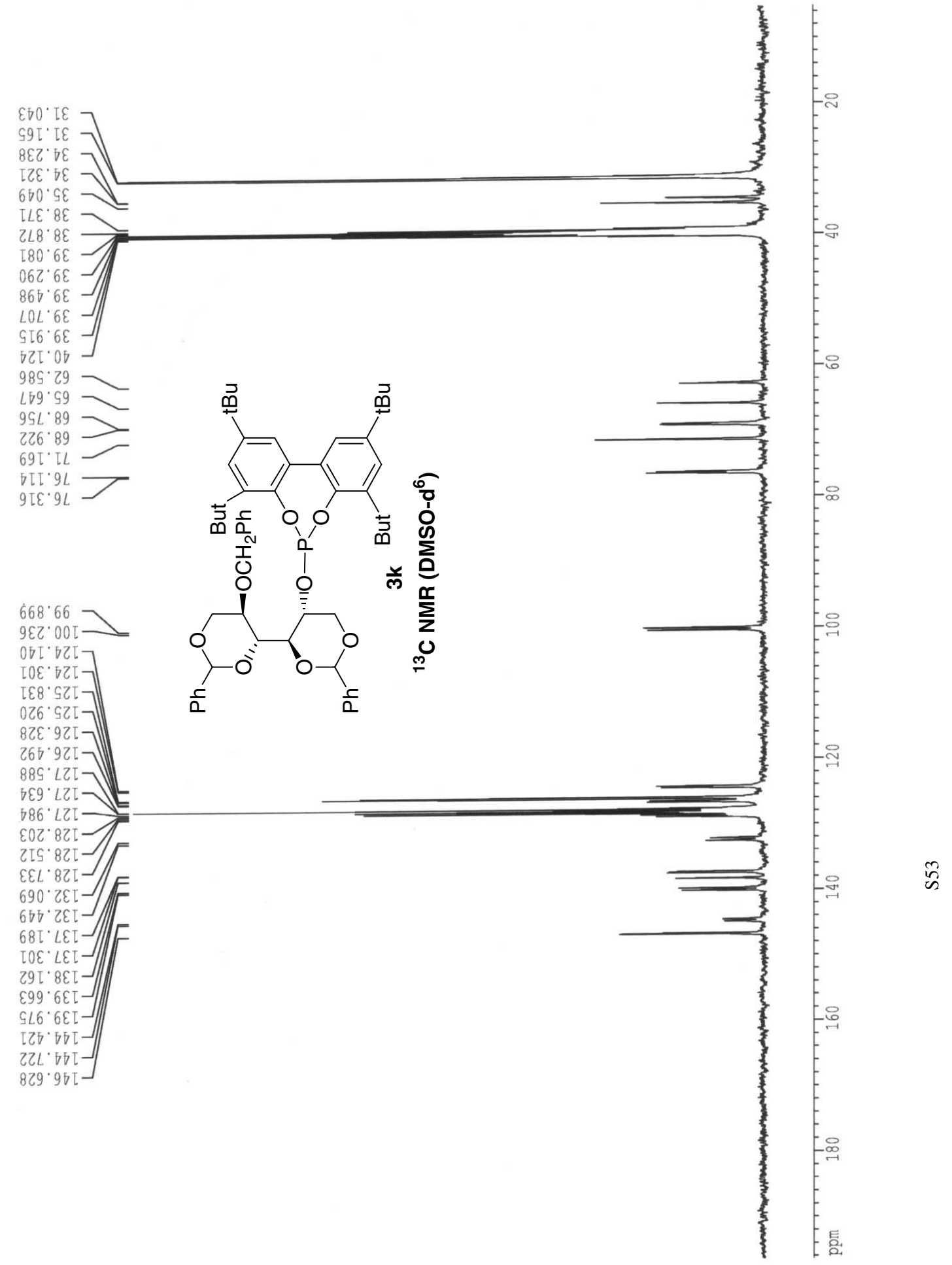




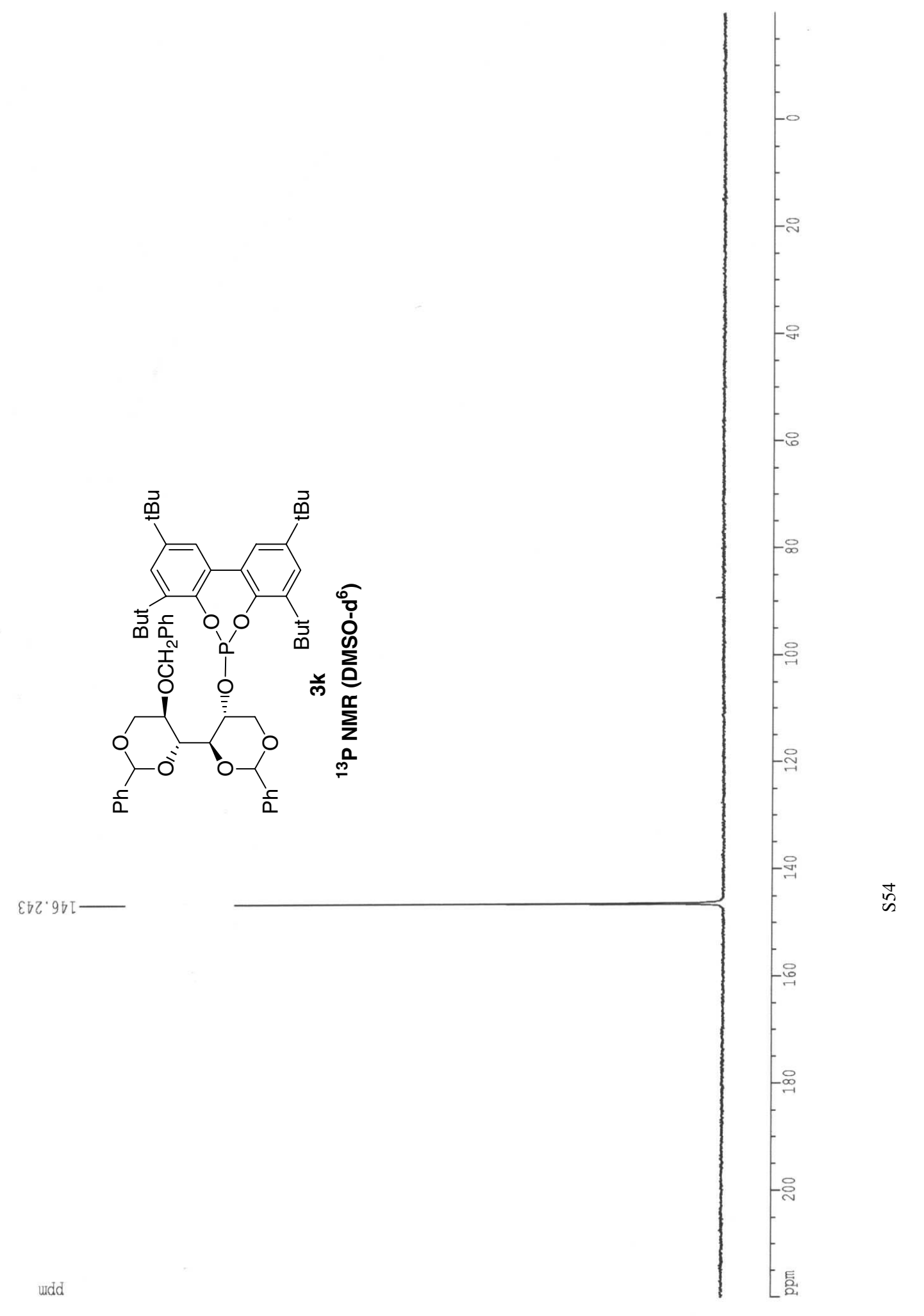


